\title{
Novel Diethynylcarbazole Macrocycles: Synthesis and Optoelectronic Properties
}

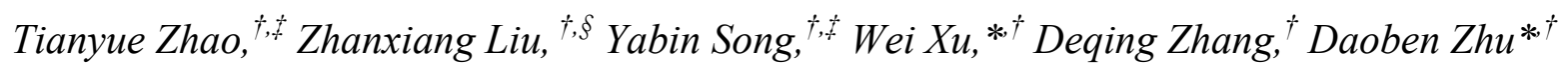

Beijing National Laboratory for Molecular Science, CAS Key Laboratory of Organic Solids, Institute of Chemistry, Chinese Academy of Sciences, and Graduate School of Chinese Academy of Sciences, Beijing 100080, P. R. China

Table of Contents

General Experimental Methods

NMR spectra 
$8 a$

S29

8b

9a

S33

9b

S36

10a

S38

10b

S40

11a

S42

11b

S44

12

S46

13

S48

14

S50

Cyclic voltammetry curves

S52

14

S52

1b

S52

2b

S52

HRMS spectra

S52

4a

S53

4b

S54

$5 b$

S55

6a

S56

6b

S57

$7 a$

S58

7b

S59

$8 a$

S60

$8 b$

S61 
General. Commercially available chemicals were used as received. Tetrahydrofuran was distilled over sodium/benzophenone prior to use. Pyridine was distilled over $\mathrm{NaOH}$.

In the Langmuir-Blogett experiments, Chloroform (spectrograde) was used for solution preparation. The LB Minitrough systems were used for isotherm determination and film deposition. The subphase was ultrapure water (resistivity: $18.2 \mathrm{M} \Omega \cdot \mathrm{cm}^{-1}$ ). All the $\Pi-A$ isotherms were recorded 15 min after spreading to allow solvent evaporation. The barrier speed was $10 \mathrm{~mm} \cdot \mathrm{min}^{-1}$. The LB monolayers were transferred onto substrates in the vertical mode (upward stroke). For the AFM measurement, the film was transferred onto freshly cleavage mica. Samples for spectroscopic studies were deposited onto quartz substrates which were cleaned with an ultrasonicator in distilled water, chloroform and isopropyl alcohol for $20 \mathrm{~min}$ in succession. For the photoelectrical measurement, indium tin oxide (ITO) glass slides with a sheet resistance of $20 \Omega$ per square were used. The glasses were cleaned with an ultrasonic bath in distilled water and isopropyl alcohol for $20 \mathrm{~min}$ in succession.

Cyclic voltammetry (CV) was recorded on a voltammetric analyzer. A three-electrode configuration comprising a platinum working electrode, a platinum wire counter electrode and an Ag wire reference electrode was used. For each compound, the platinum working electrode were covered by one thin-layer film of 14, 1b, 2b or PCBM, respectively. Acetonitrile (spectrograde) was used to prepare the solution containing tetrabutylammonium perchlorate $(0.1 \mathrm{M})$ as a supporting electrolyte. The scan rates of $\mathbf{1 4}, \mathbf{1 b}$, 
2b and PCBM were all $100 \mathrm{mV} \cdot \mathrm{s}^{-1}$. All potentials reported are referenced versus $\mathrm{Ag}$ wire. $\mathrm{N}_{2}$ was bubbled to remove oxygen from the electrolyte solutions in the electrochemical cell.

The photoelectrochemical measurements were recorded on a voltammetric analyzer with a $500 \mathrm{~W}$ halogen lamp as the light soure. The intensities of incident beams were checked by a power and energy meter. The IR light was filtered throughout the experiments to protect the electrodes from heating.

\section{NMR spectra.}



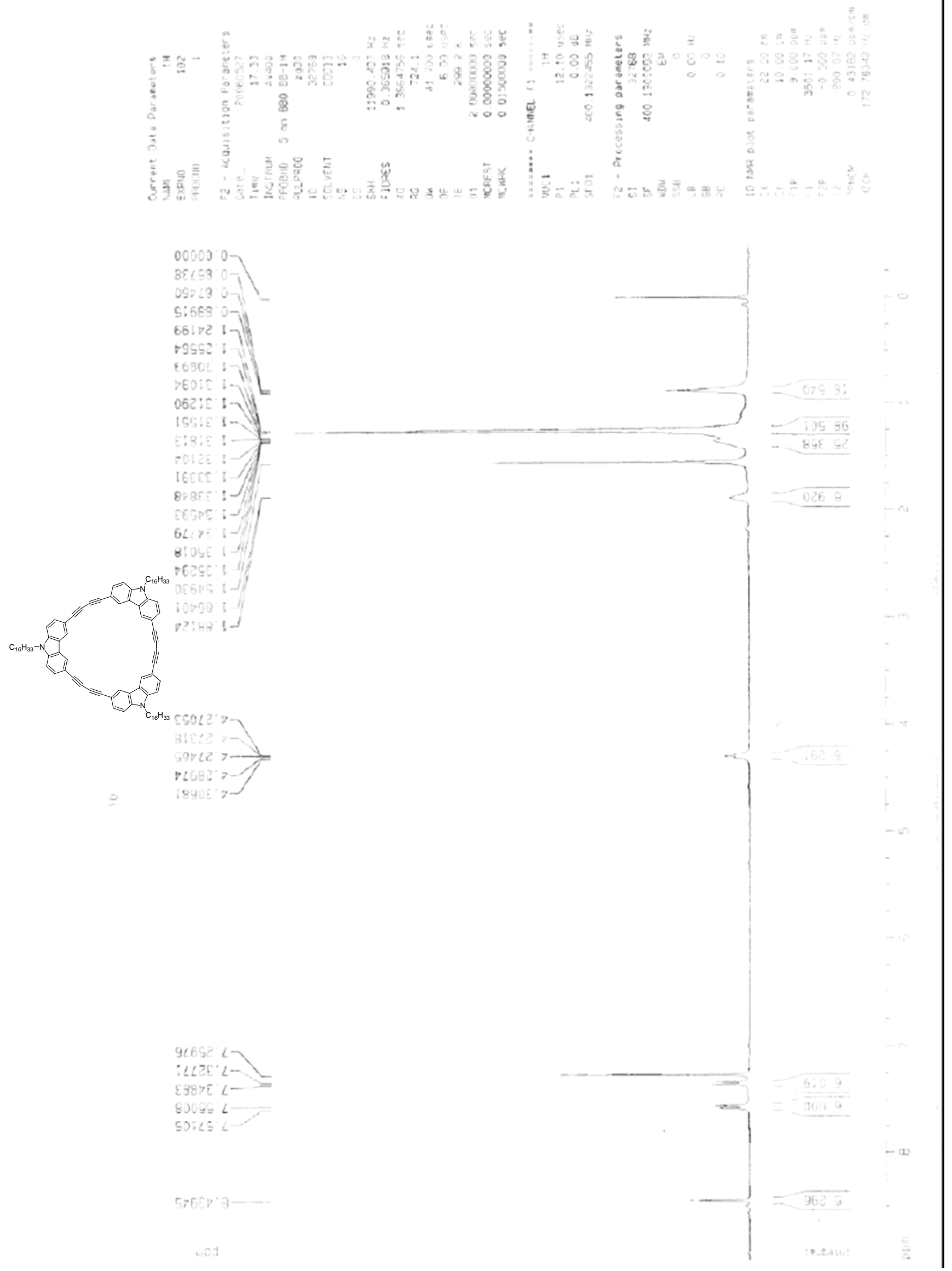

Figure S1: ${ }^{1} \mathrm{H}$ NMR spectra of $\mathbf{1 b}$ 


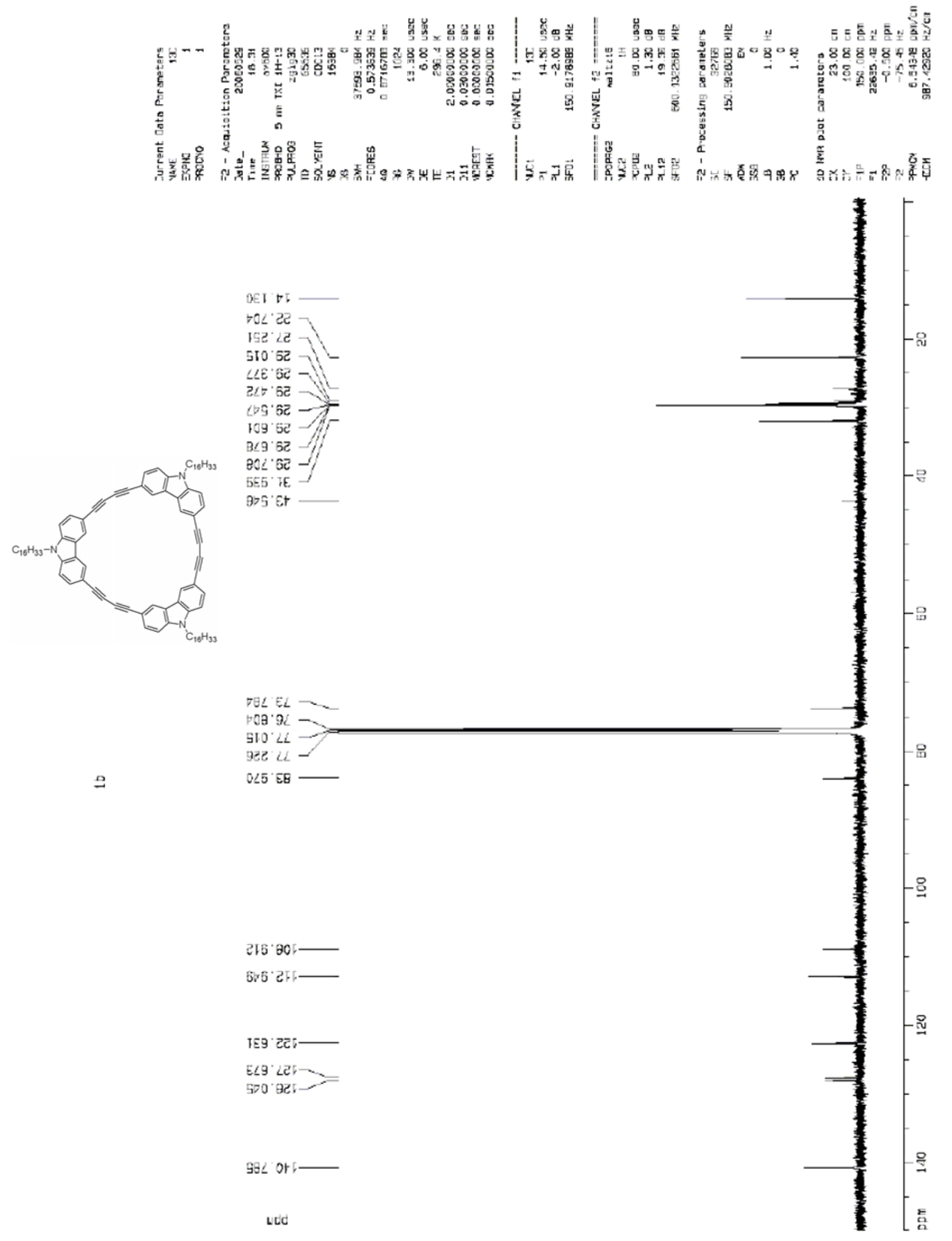

Figure S2: ${ }^{13} \mathrm{C}$ NMR spectra of $\mathbf{1 b}$ 

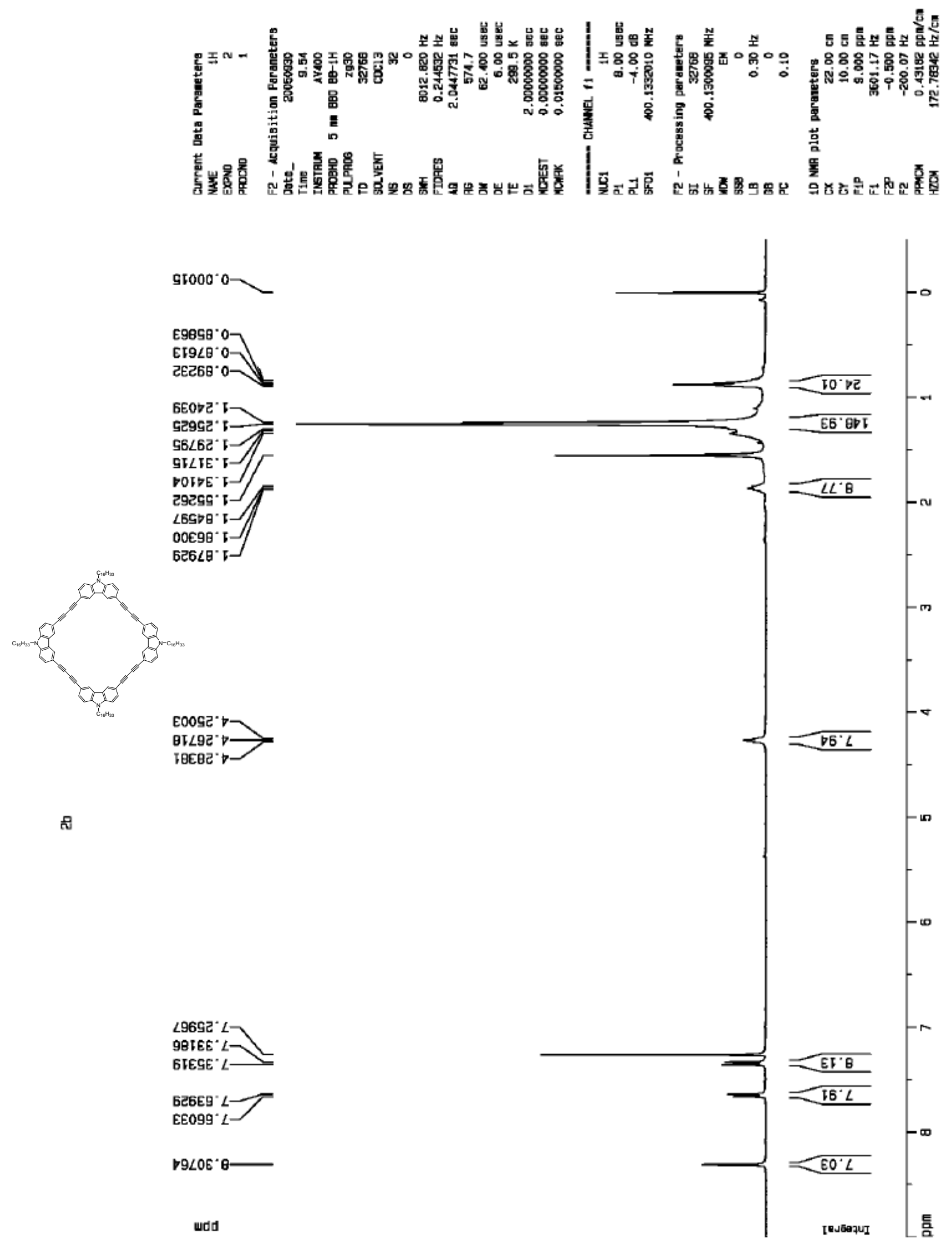

Figure S3: ${ }^{1} \mathrm{H}$ NMR spectra of $\mathbf{2 b}$ 

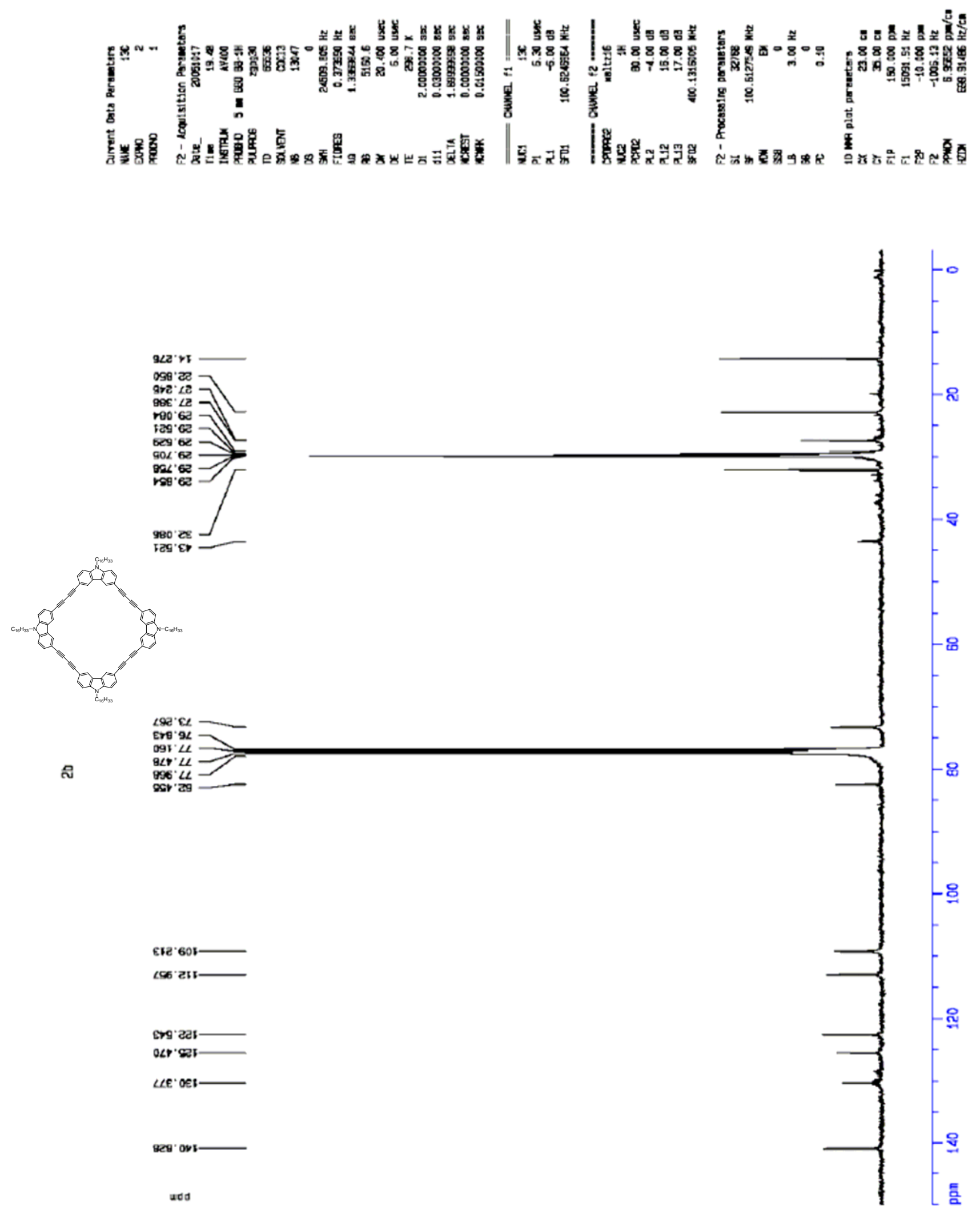

Figure S4: ${ }^{13} \mathrm{C}$ NMR spectra of $\mathbf{2 b}$ 

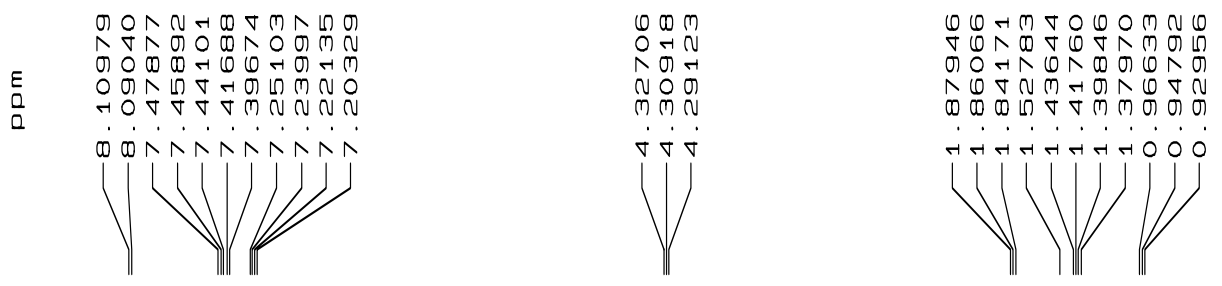

Current Data Parameters

NAME

EXPNO

PROCNO

F2 - Acquisition Parameters

Date_ 20060520

Time $\quad 11.18$

INSTRUM AVAOO

PPOBHD 5 ח ח BBO BB-IH

PULPPOG 2030

TD 32768

SOLENT COCI3

NS 16

DS 0

5WH $\quad 11990.407 \mathrm{~Hz}$

FIDRES $\quad 0.365918 \mathrm{~Hz}$

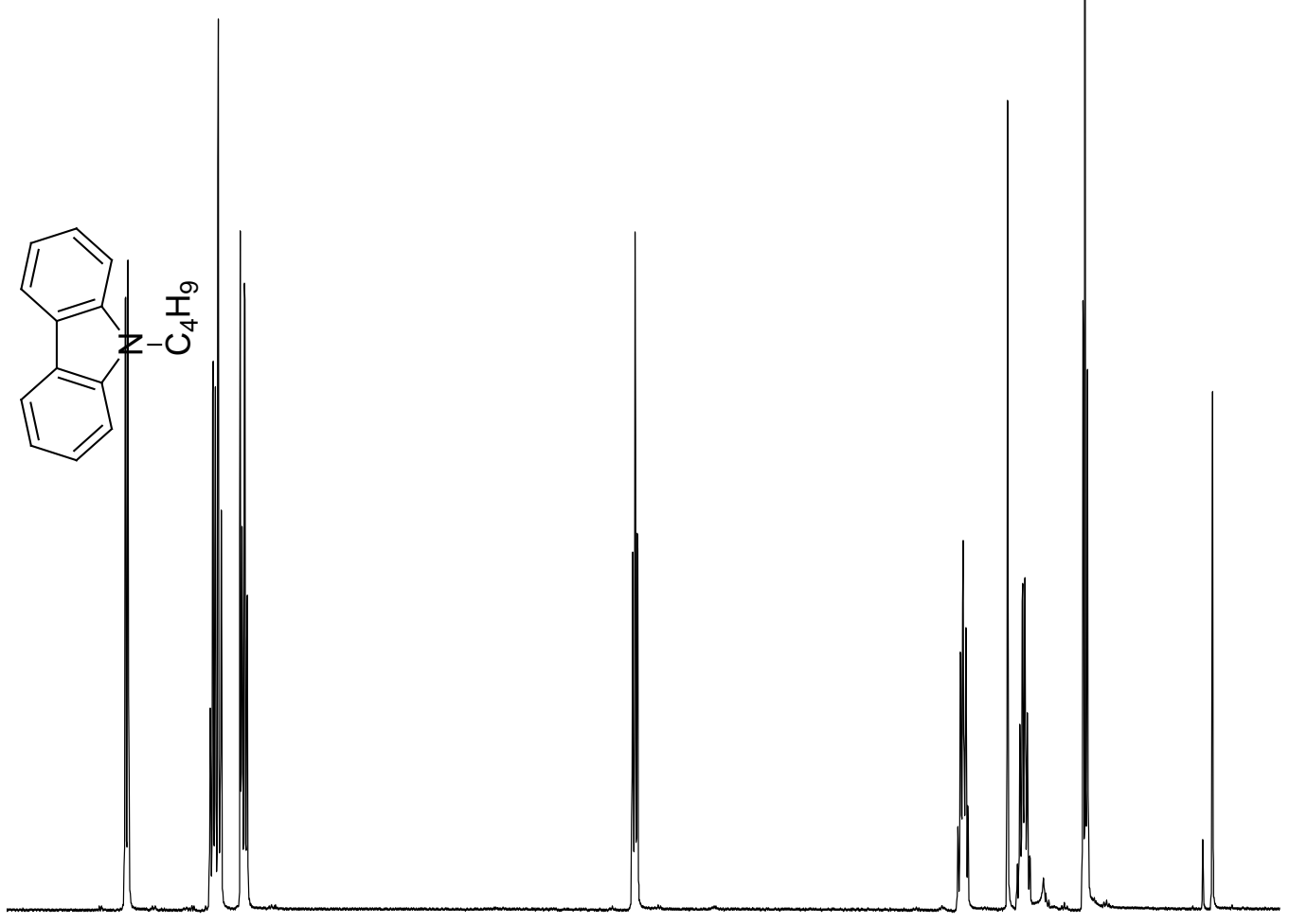

AQ $\quad 1.3664756 \mathrm{sec}$

HG 512

DN $\quad 41.700$ usec

DE $\quad 6.00$ usec

TE $\quad 298.8 \mathrm{~K}$

$01 \quad 5.00000000 \mathrm{sec}$

MCREST $\quad 0.00000000 \mathrm{sec}$

MCWRK $\quad 0.01500000 \mathrm{sec}$

z:z:z:=: CHANEL $f 1$ =:z=:z:=

NCI IH

P1 $\quad 12.10$ usec

PL1 $\quad 0.00 \mathrm{~dB}$

SF01 400.1322455 MHz

F2 - Processing parameters

SI $\quad 32768$

SF $\quad 400.1300125 \mathrm{MHz}$

NOW EM

SSB 0

LB $\quad 0.30 \mathrm{~Hz}$

$\begin{array}{lr}G B & 0 \\ P C & 0.10\end{array}$

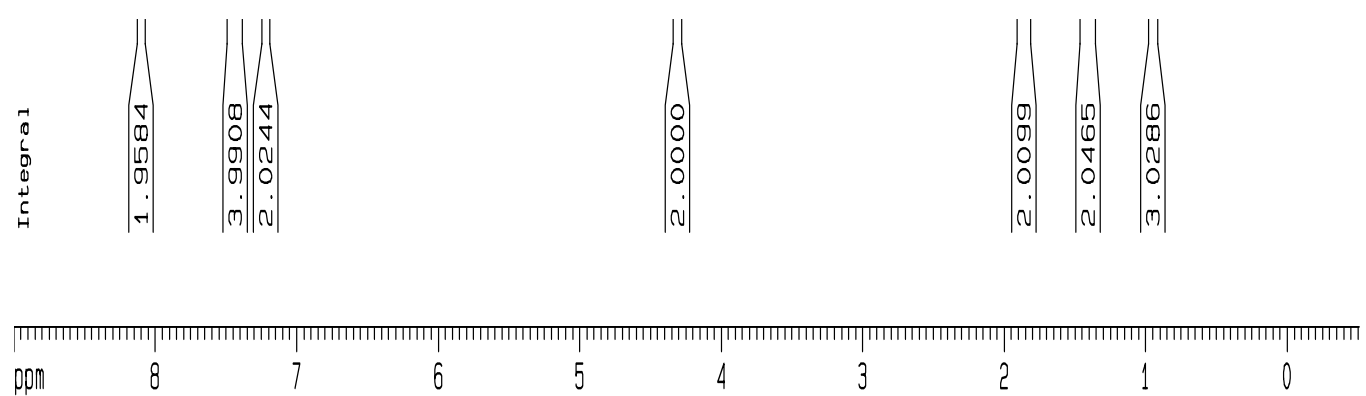

10 NMR plot paraneters

CX $\quad 22.00 \mathrm{~cm}$

CY $\quad 10.00 \mathrm{~cm}$

F.P $\quad 9.000 \mathrm{ppm}$

F1 $\quad 3601.17 \mathrm{~Hz}$

F.P $\quad-0.500 \mathrm{ppm}$

F2 $\quad-200.07 \mathrm{~Hz}$

PPMCM $\quad 0.43182 \mathrm{pDm} / \mathrm{cm}$

HZCM $\quad 172.78342 \mathrm{~Hz} / \mathrm{cm}$

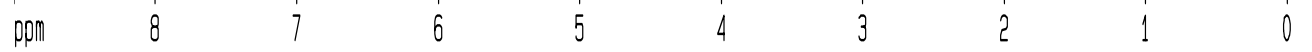

Figure S5: ${ }^{1} \mathrm{H}$ NMR spectra of 3a 


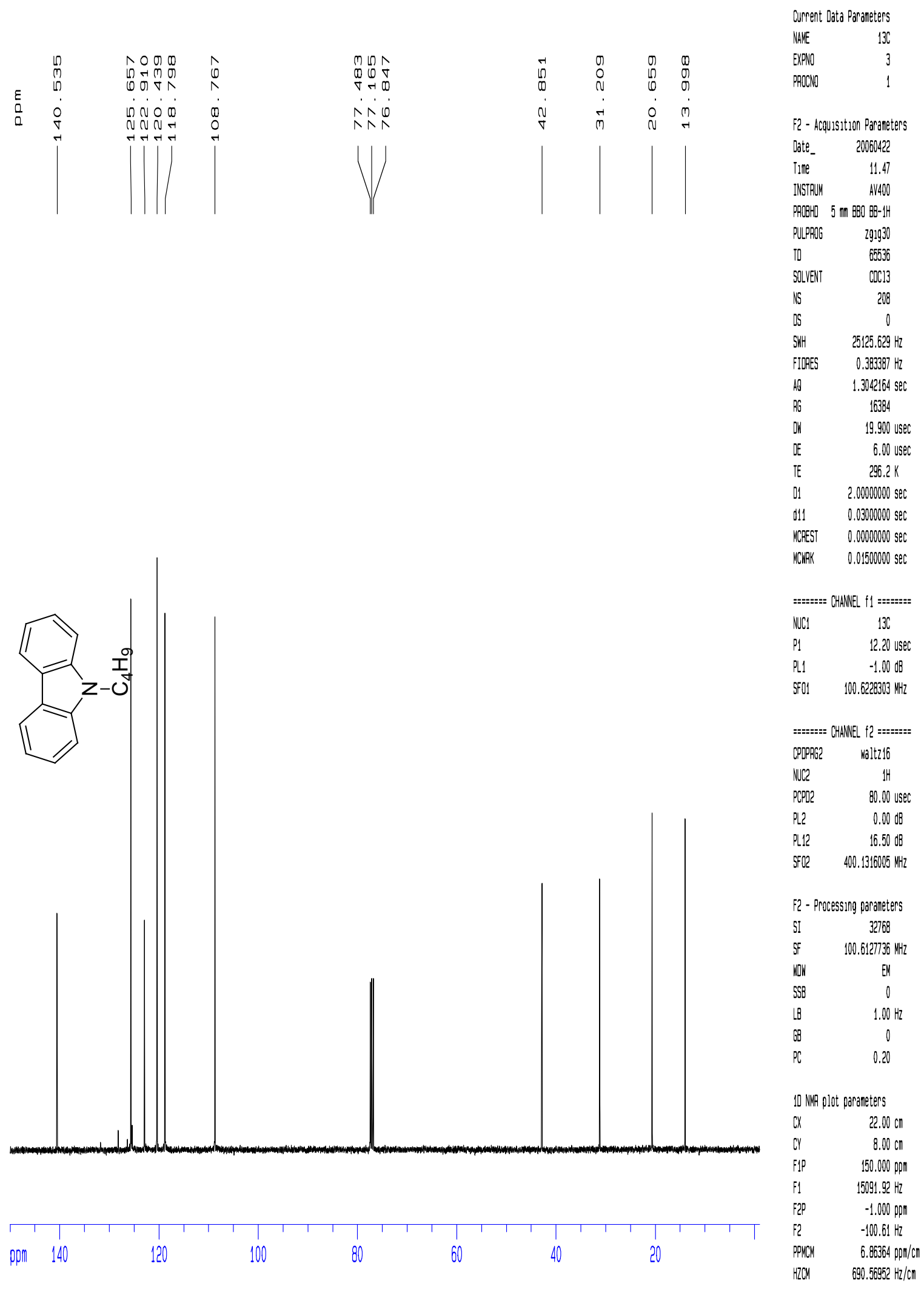

Figure S6: ${ }^{13} \mathrm{C}$ NMR spectra of 3a 

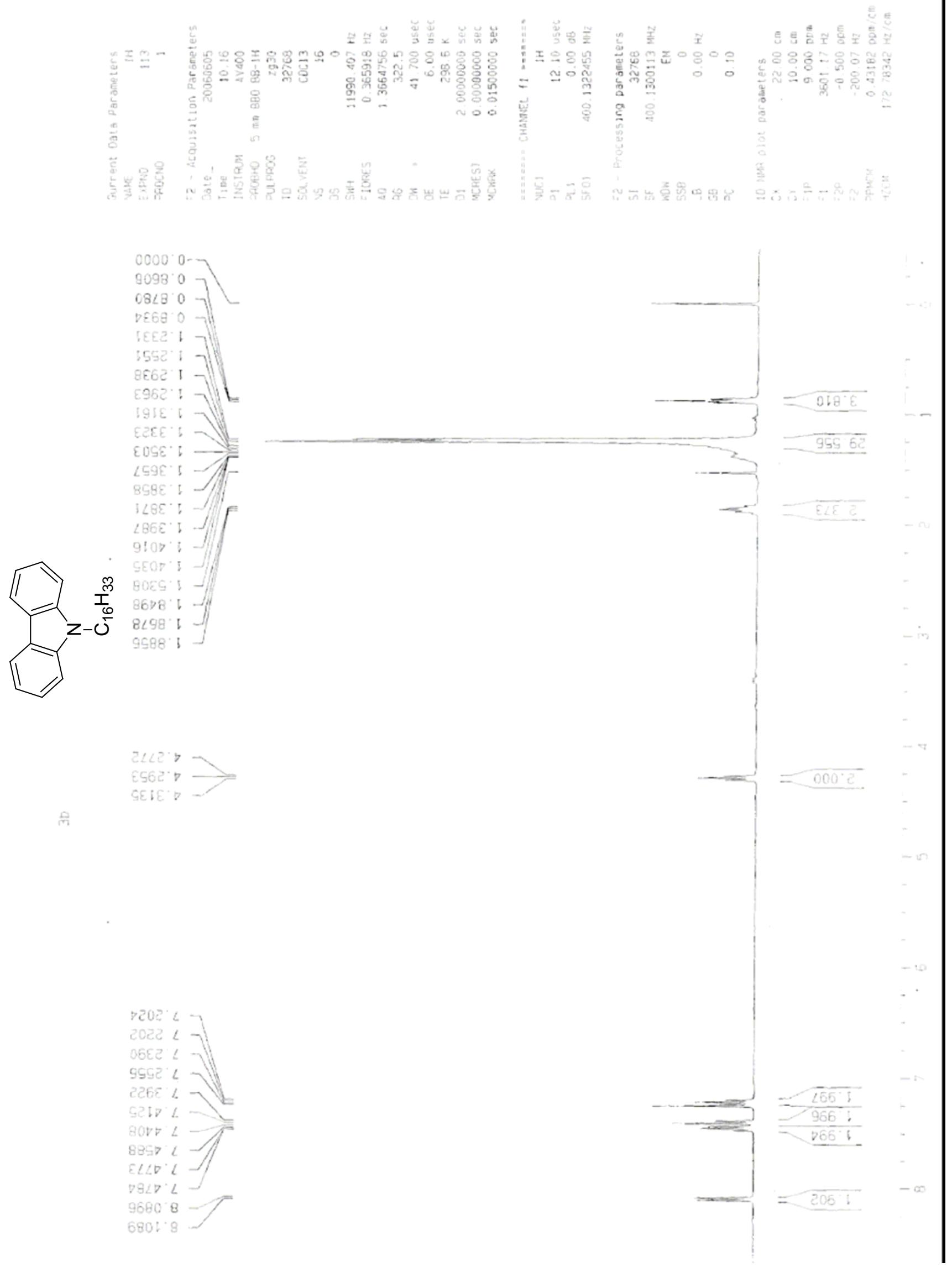

Figure S7: ${ }^{1} \mathrm{H}$ NMR spectra of $\mathbf{3 b}$

SI 

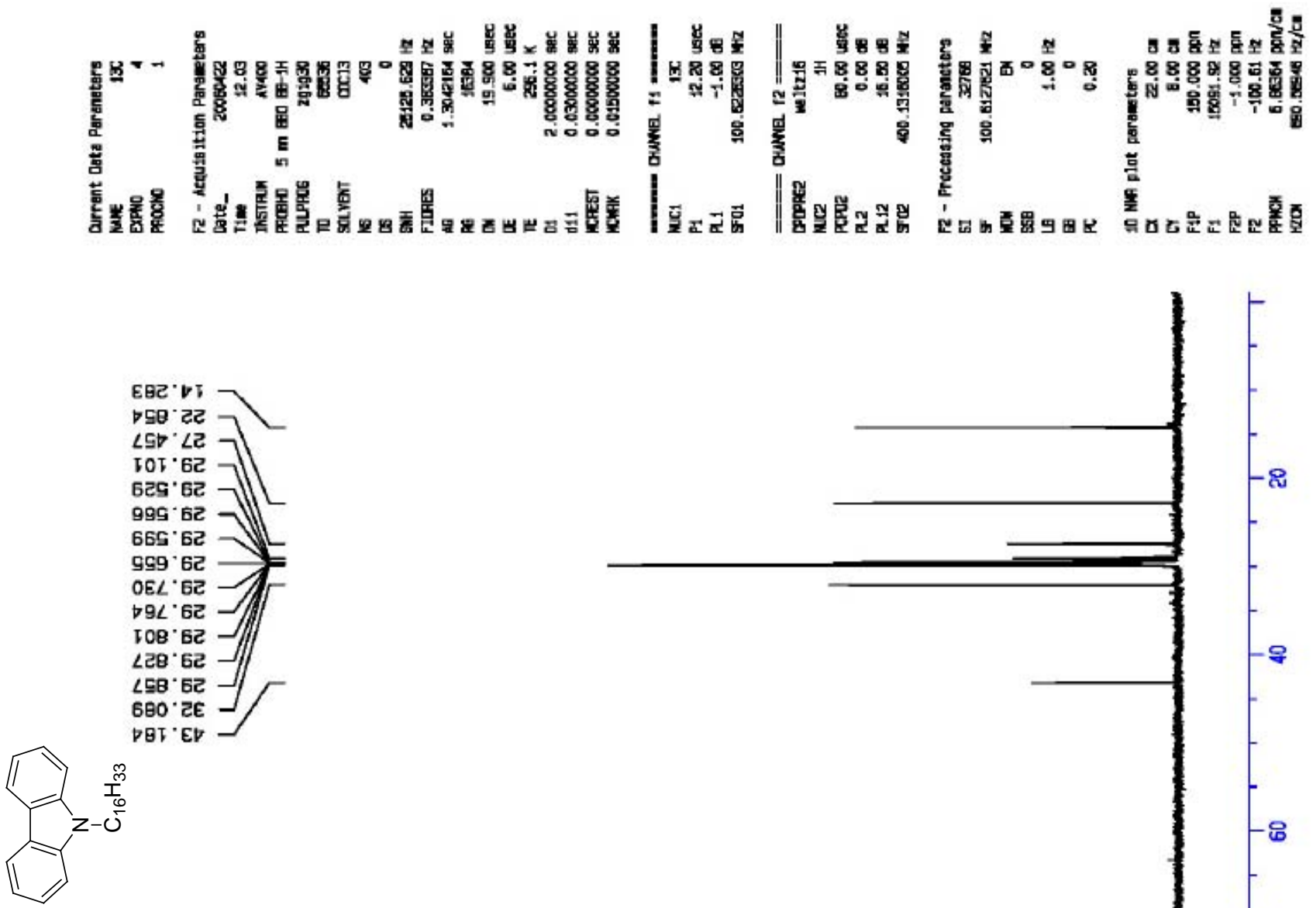

IVB. 92

$69 T^{\circ} \cdot \angle L$

กิ

$9 L b \cdot \angle L->$

$09 L^{\circ}$ BOV

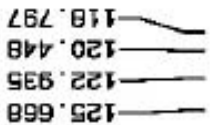

B99.

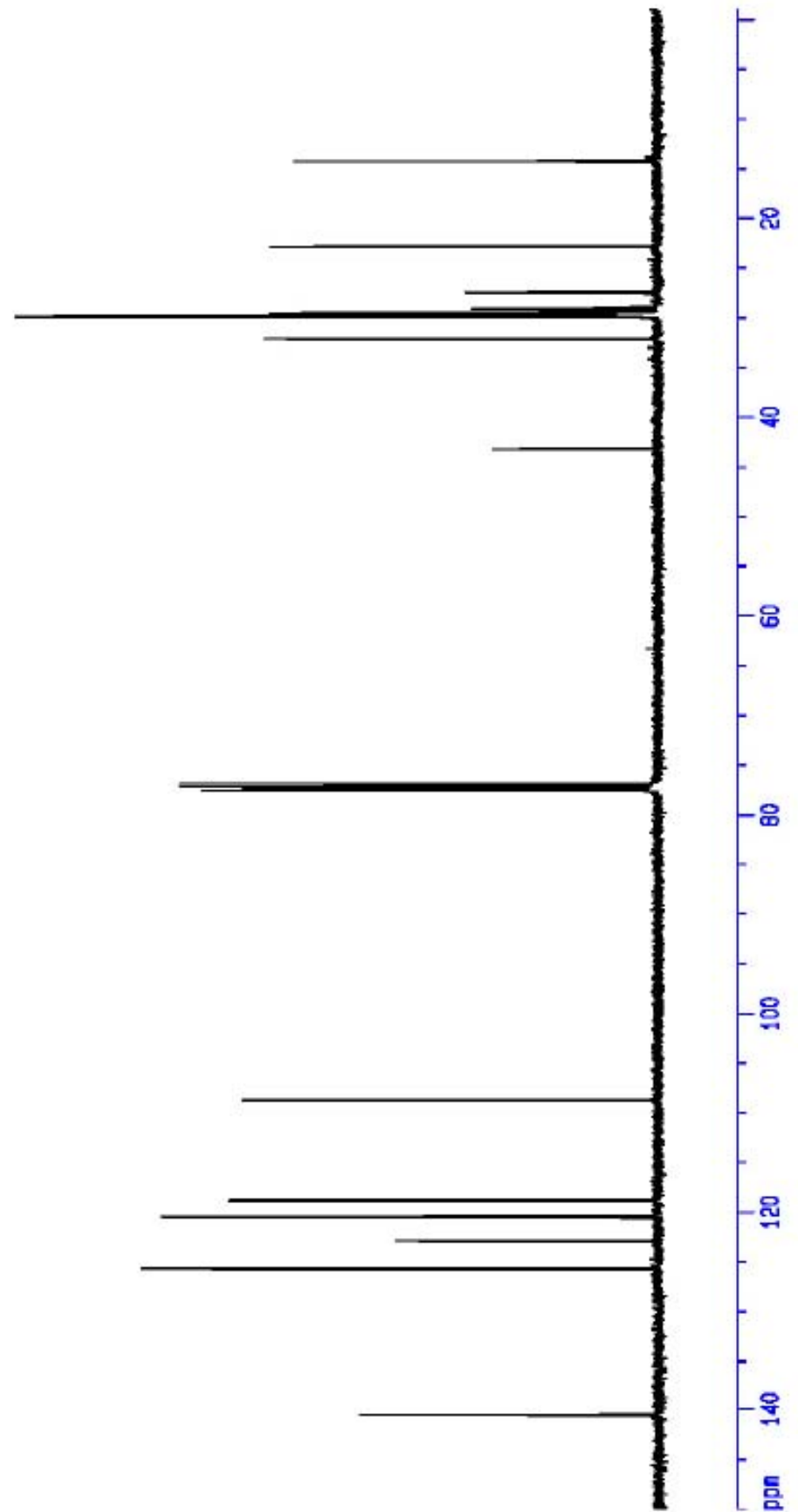

wdd

Figure S8: ${ }^{13} \mathrm{C}$ NMR spectra of $\mathbf{3 b}$ 


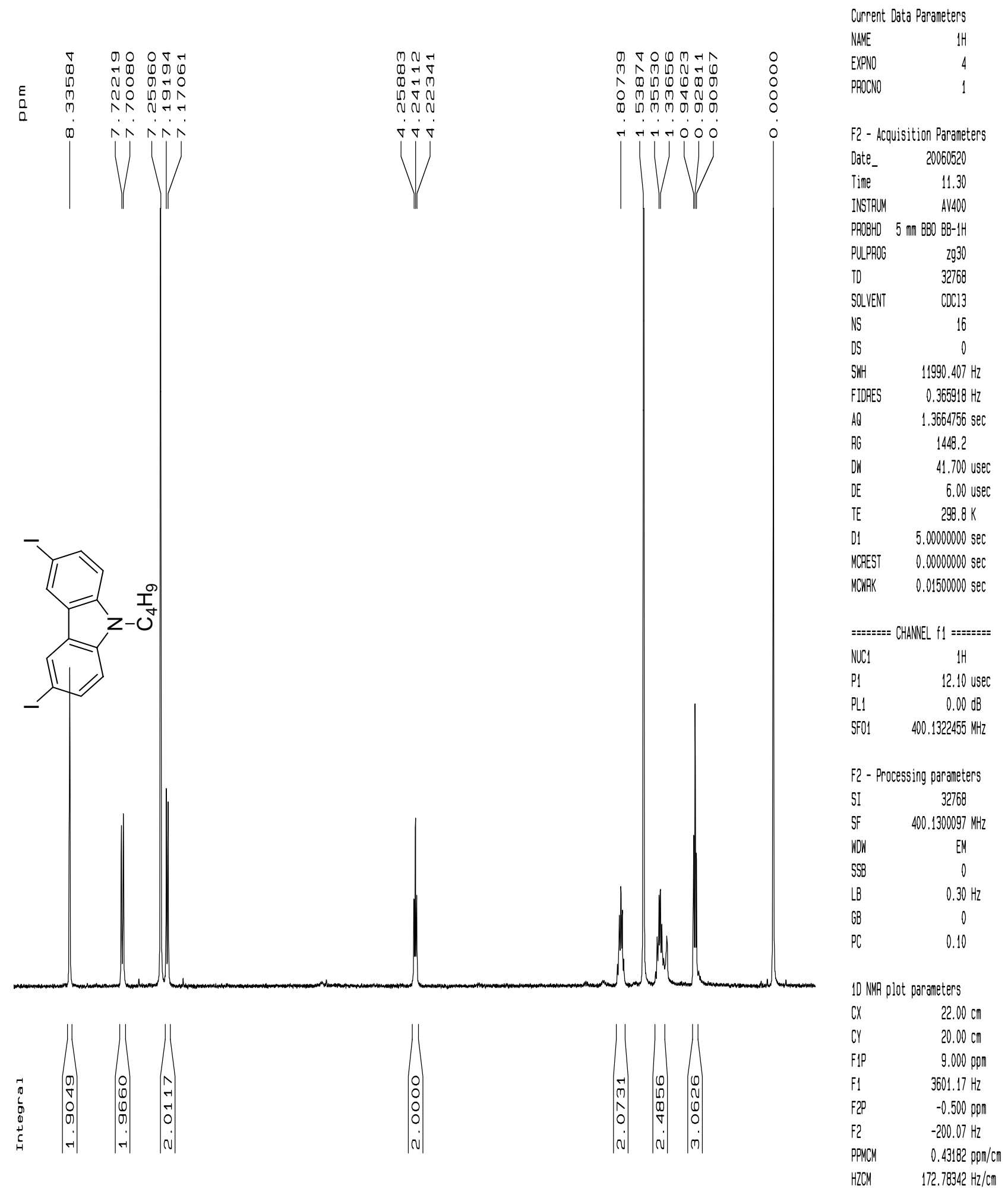

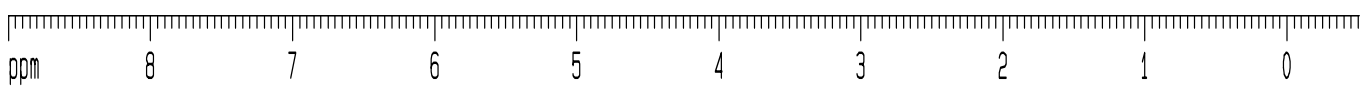

Figure S9: ${ }^{1} \mathrm{H}$ NMR spectra of $4 a$ 

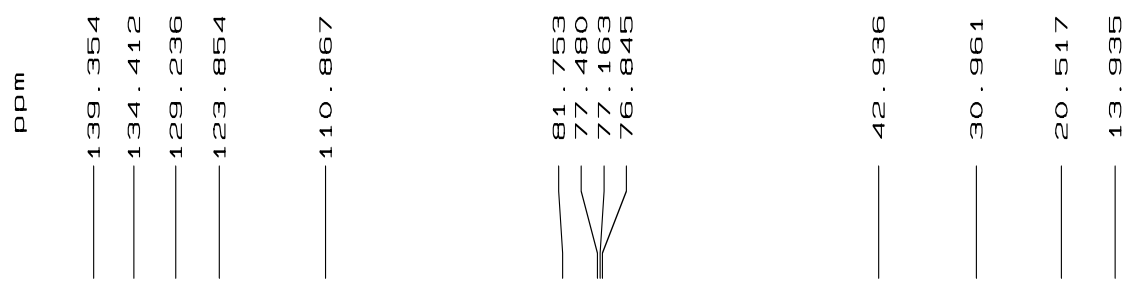

uurent Data Parameters

VAME $13 \mathrm{C}$

$\begin{array}{ll}\text { EXPNO } & 5 \\ \text { JPOCNO } & 1\end{array}$

:2 - Acquisition Paraneters

Jate_ 200600422

Tine $\quad 11.32$

INSTRUM AV400

3ROOHO 5 In 880 88-1H

गULPPOG 291930

To 65536

SOLENT COCI3

US 222

350

3 3 . $25125.629 \mathrm{~Hz}$

IIOPES $\quad 0.383387 \mathrm{~Hz}$

AO $\quad 1.3042164 \mathrm{sec}$

$36 \quad 11585.2$

JH $\quad 19.900$ usec

JE $\quad 6.00$ usec

TE $296.3 \mathrm{~K}$

$31 \quad 2.00000000 \mathrm{sec}$

$1110.03000000 \mathrm{sec}$

ICREST $0.00000000 \mathrm{SeC}$

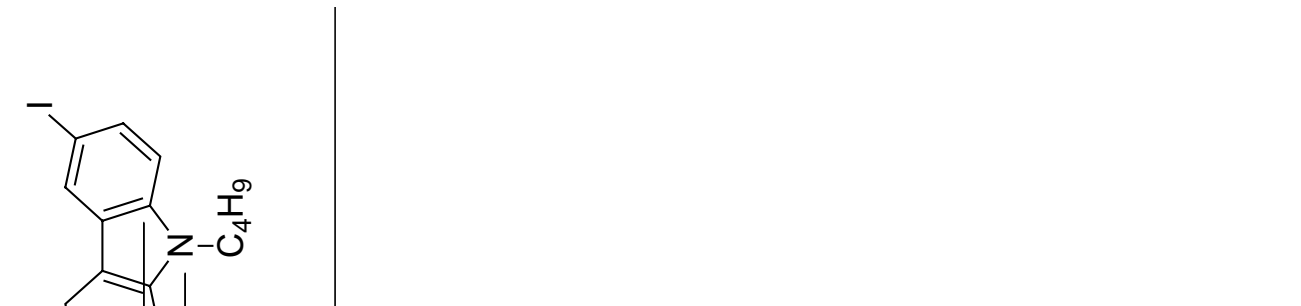

ICWRK $0.01500000 \mathrm{sec}$

=:z=:=: CHANEL $f 1$ =:e=:=:

\begin{tabular}{|c|c|}
\hline ICI & $13 C$ \\
\hline 9 & $12.20 \mathrm{usec}$ \\
\hline & $-1.00 \mathrm{~dB}$ \\
\hline & $100.6228303 \mathrm{MHz}$ \\
\hline$=:=:$ & AANELL f? =:=:=:= \\
\hline PRG2 & Maltzit. \\
\hline & 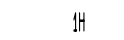 \\
\hline 02 & 80.00 usec \\
\hline & $0.00 \mathrm{~dB}$ \\
\hline & $16.50 \mathrm{~dB}$ \\
\hline & 400.1316005 WH. \\
\hline
\end{tabular}

:2 - Processing paraneters

3I $\quad 32768$

jF $\quad 100.6127705 \mathrm{HHz}$

NON EX

358

B $\quad 1.00 \mathrm{~Hz}$

$38 \quad 0$

$\begin{array}{ll}x & 0.20\end{array}$

10 NWR plot paraneters

xX $\quad 22.00 \mathrm{~cm}$

y $\quad 8.00 \mathrm{~cm}$

:19 $\quad 150.000$ pon

$=1 \quad 15091.92 \mathrm{~Hz}$

$=2 p \quad-1.000$ pom

$\because 2 \quad-100.61 \mathrm{~Hz}$

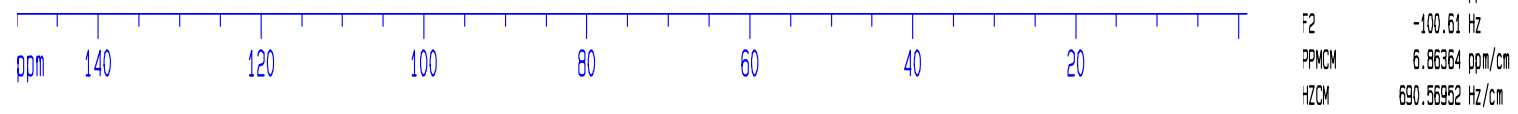

Figure S10: ${ }^{13} \mathrm{C}$ NMR spectra of $4 a$ 


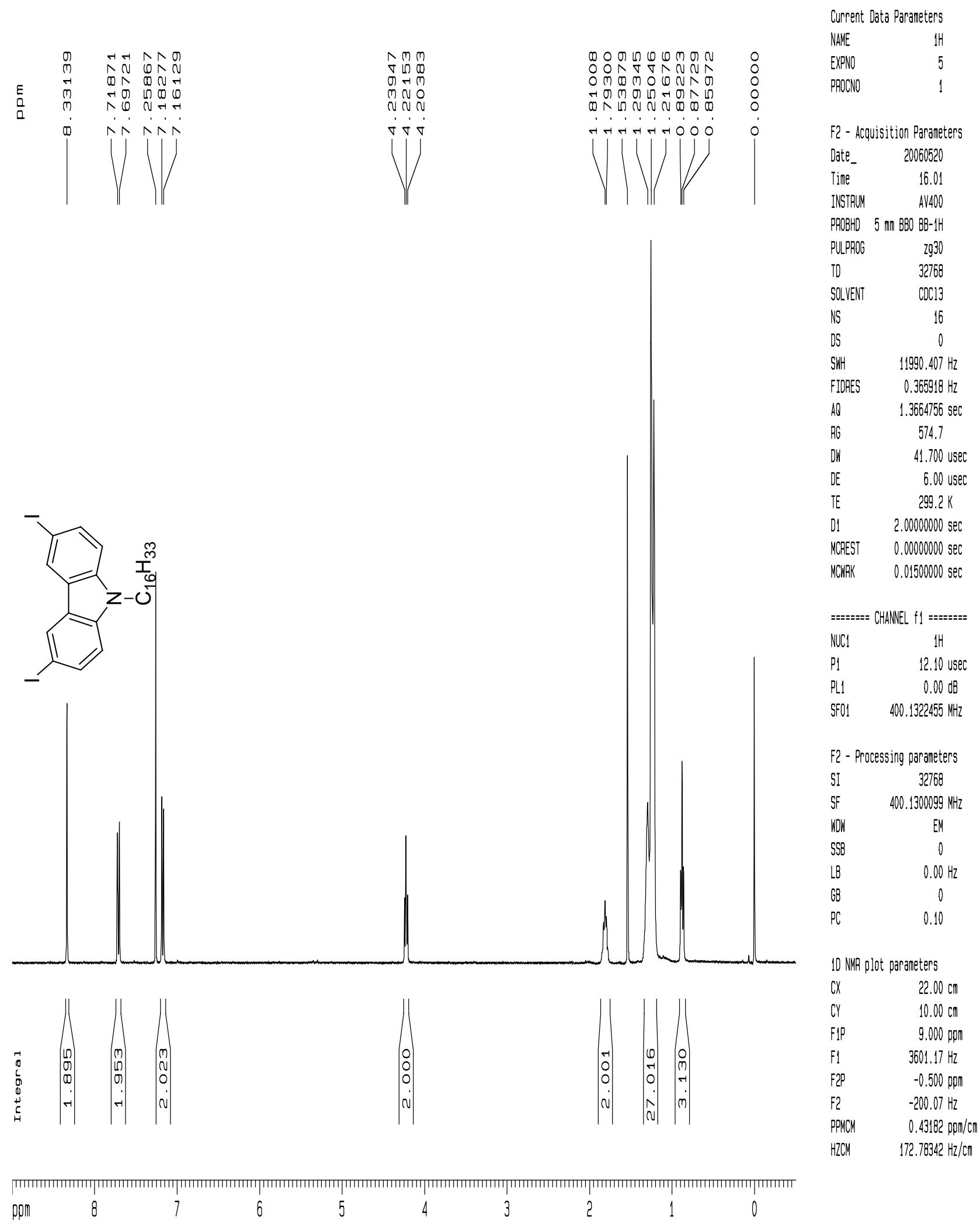

Figure S11: ${ }^{1} \mathrm{H}$ NMR spectra of $\mathbf{4 b}$ 


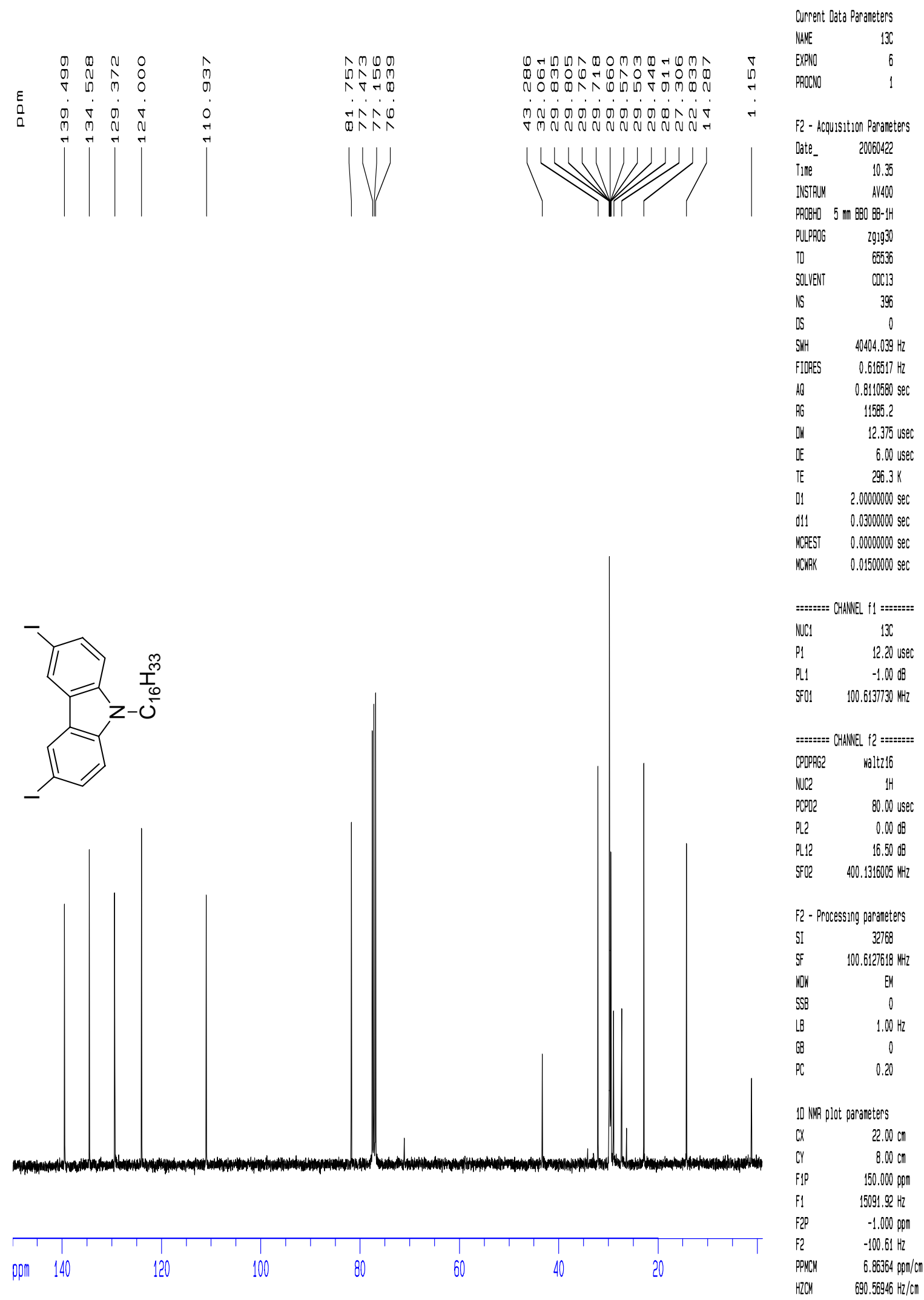

Figure S12: ${ }^{13} \mathrm{C}$ NMR spectra of $\mathbf{4 b}$ 

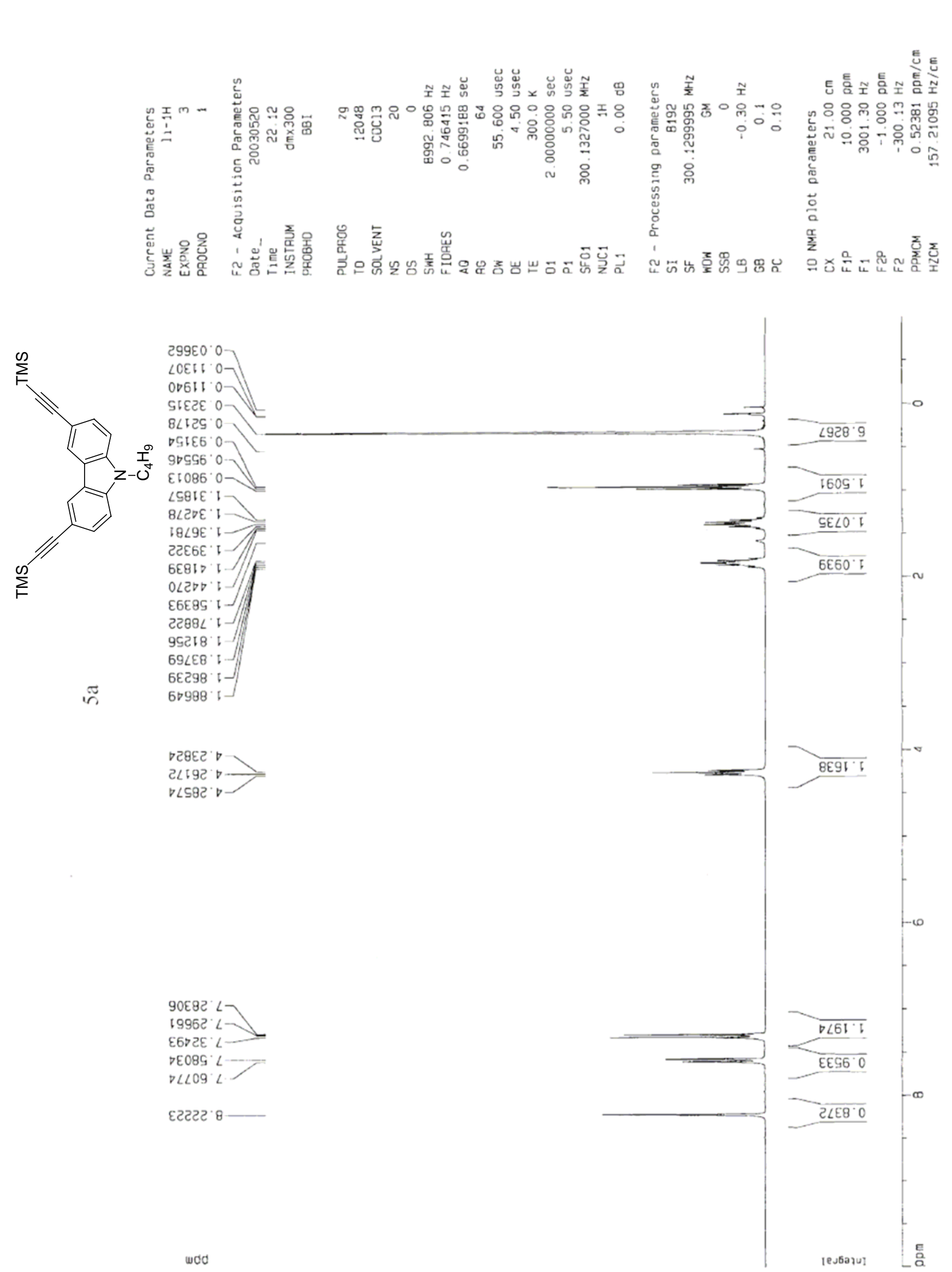

Figure S13: ${ }^{1} \mathrm{H}$ NMR spectra of $\mathbf{5 a}$ 

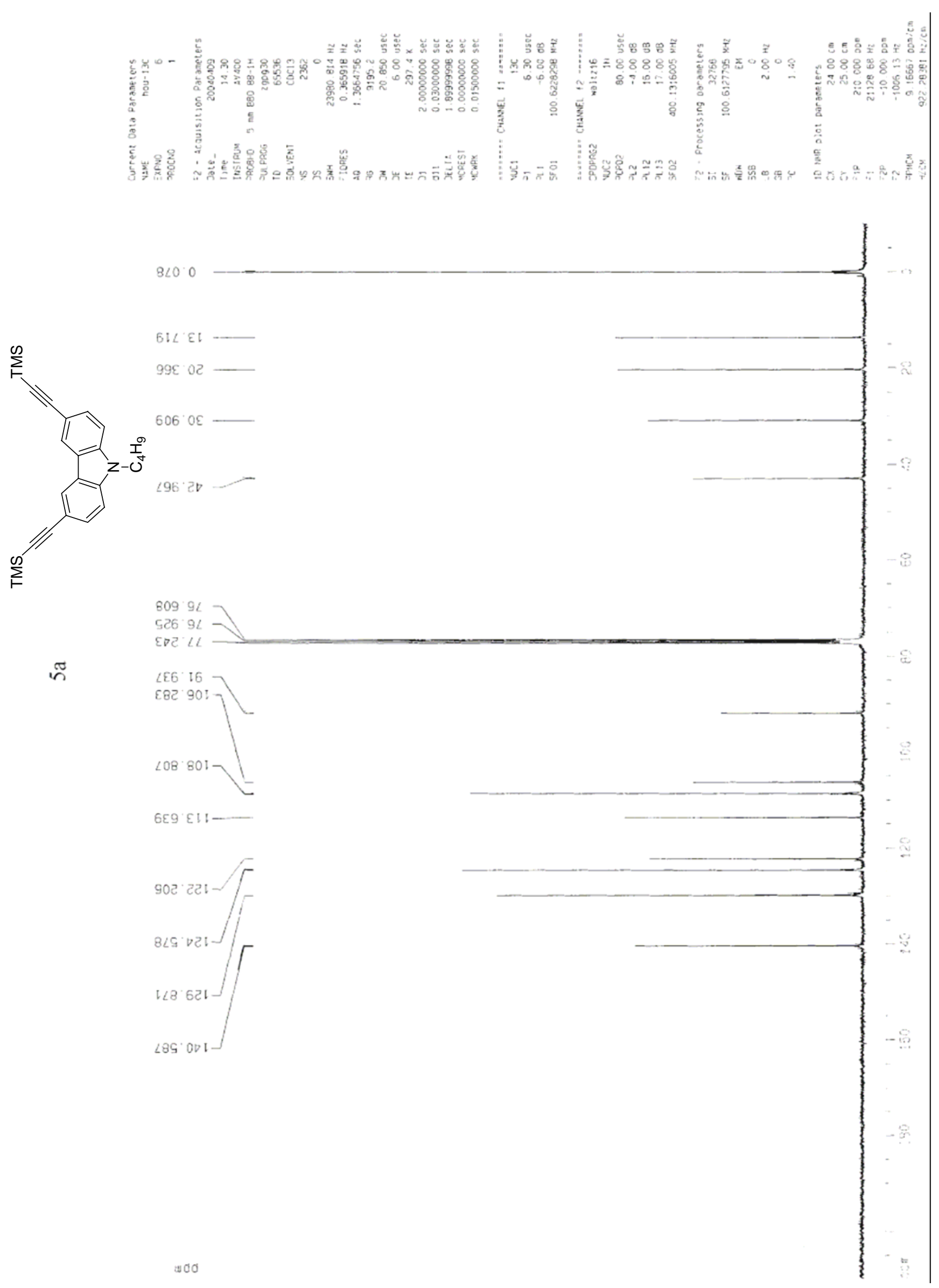

Figure S14: ${ }^{13} \mathrm{C}$ NMR spectra of 5a 

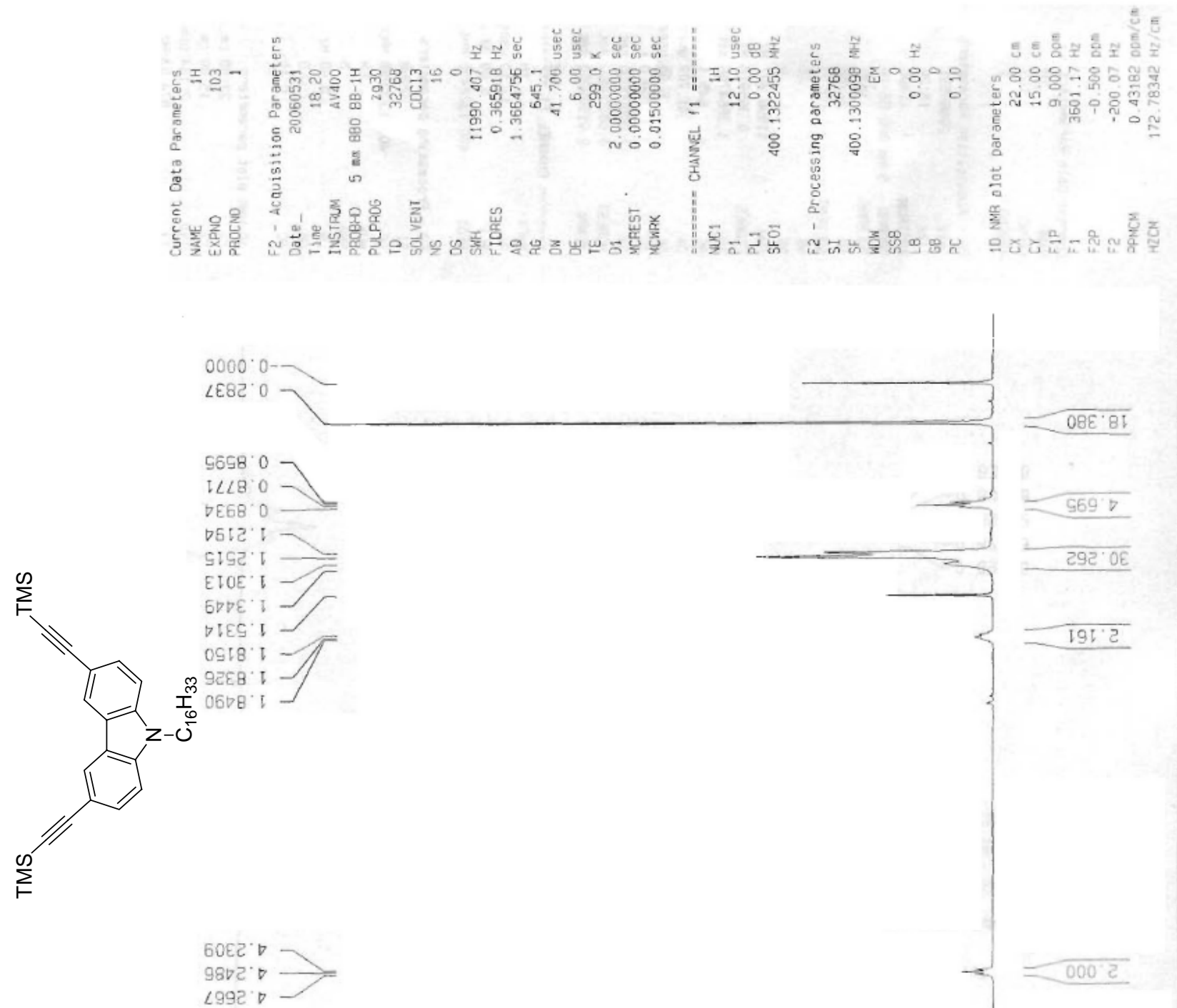

요

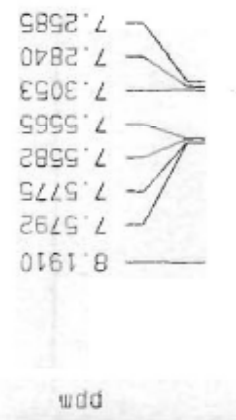

Figure S15: ${ }^{1} \mathrm{H}$ NMR spectra of $5 \mathbf{b}$ 


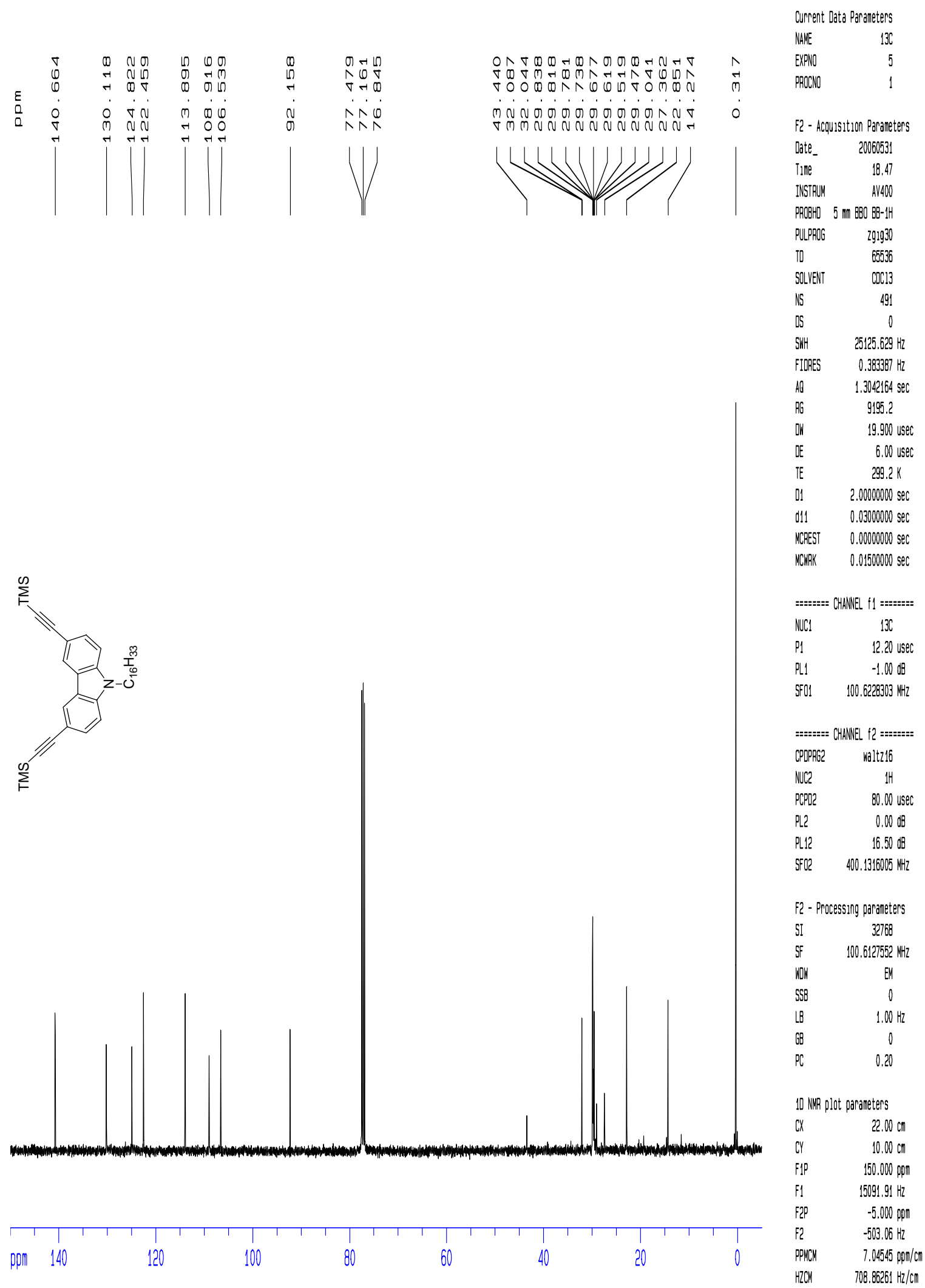

Figure S16: ${ }^{13} \mathrm{C}$ NMR spectra of $5 \mathbf{b}$ 

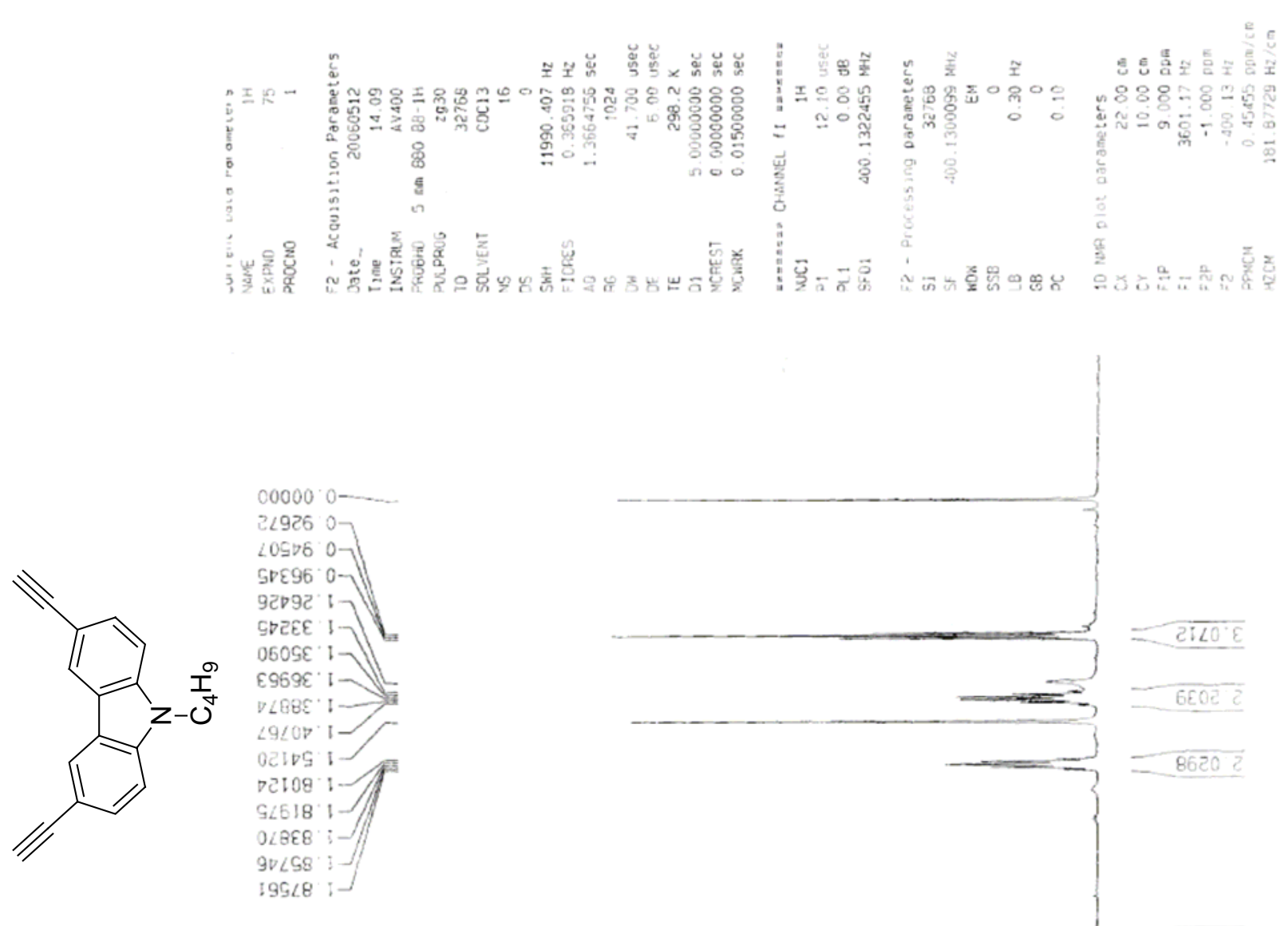

$\pi$

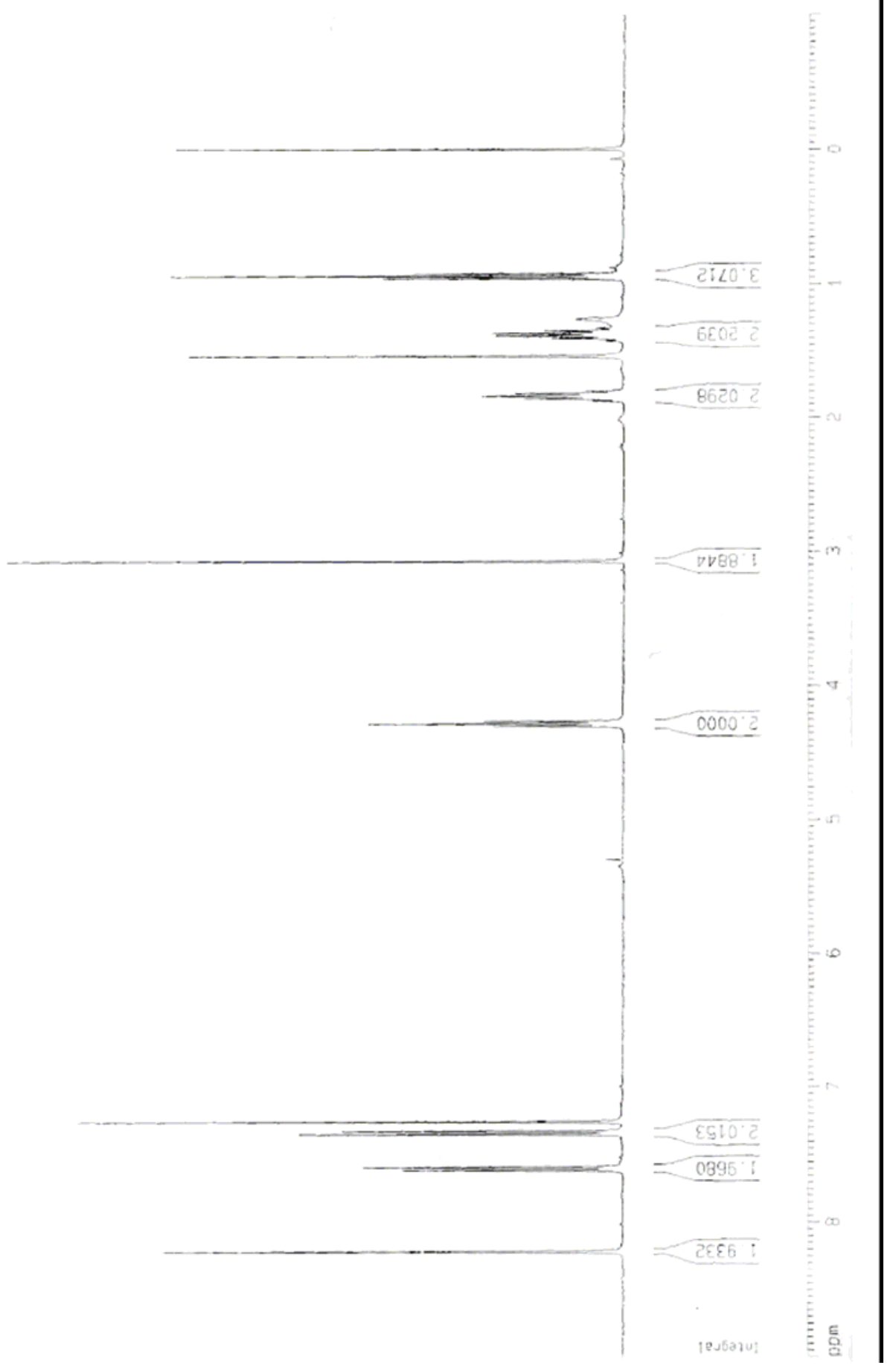

Figure S17: ${ }^{1} \mathrm{H}$ NMR spectra of $\mathbf{6 a}$ 

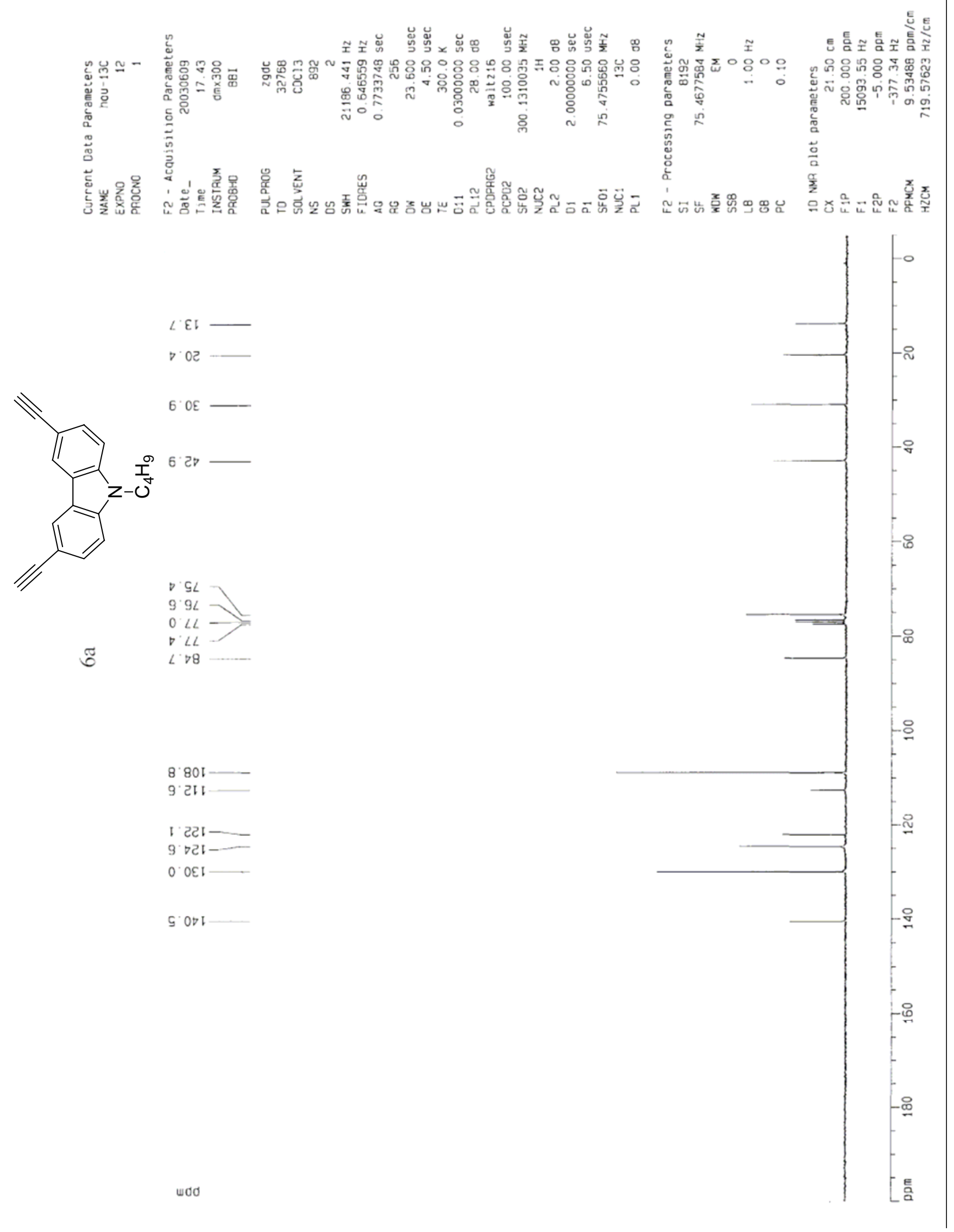

Figure S18: ${ }^{13} \mathrm{C}$ NMR spectra of $\mathbf{6 a}$ 

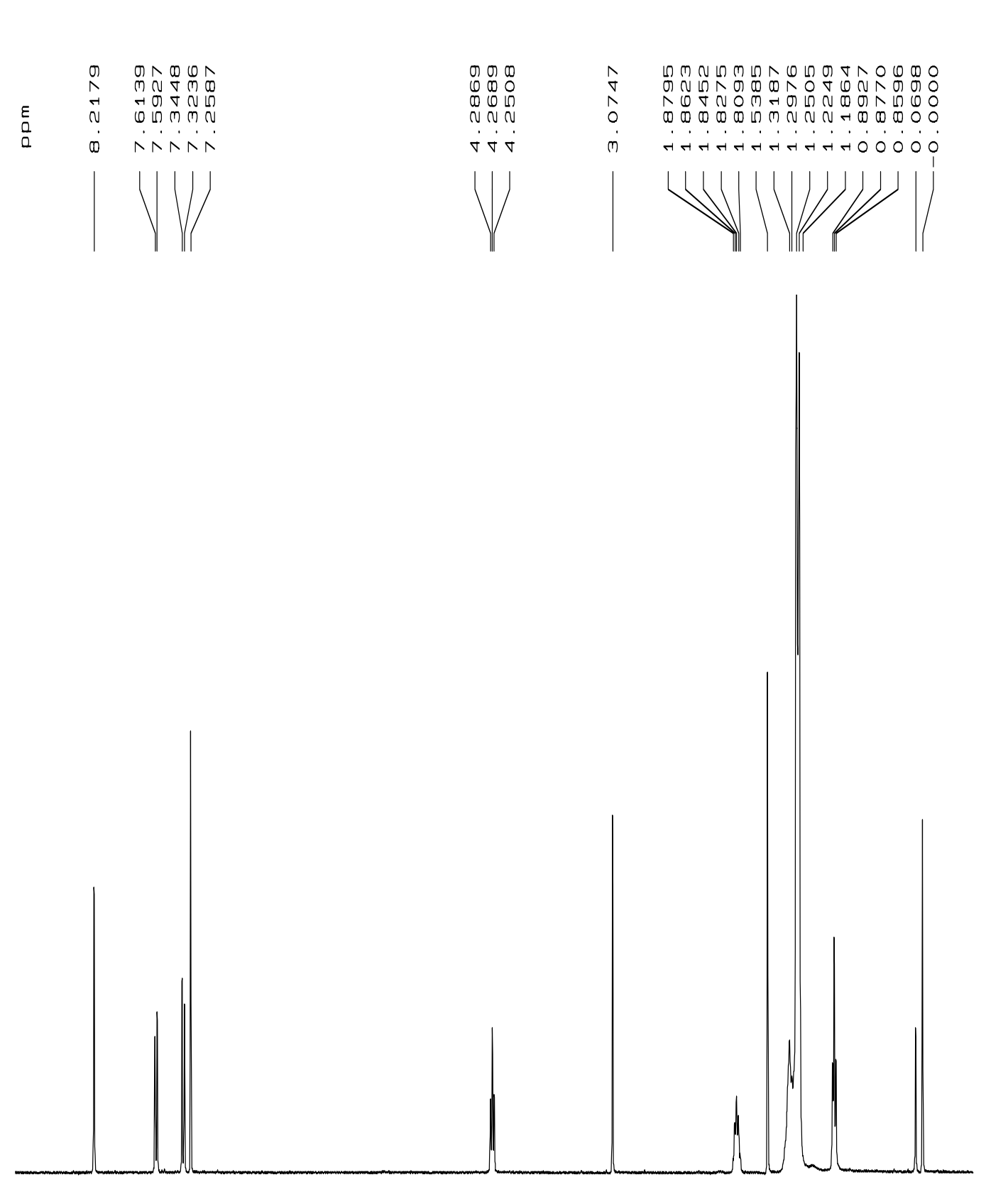

Current Data Parameters

NAME

EXPNO

PROCNO

₹2 - Acquisition Parameters

Jate_ 20060520

Time $\quad 12.17$

INSTRUM AV4OO

JROBHD 5 m $\mathrm{BBO}$ BB-1H

PULPROG 2930

TD 32768

SOLVENT COCI3

NS 16

$35 \quad 0$

SWH $\quad 11990.407 \mathrm{~Hz}$

FIDRES $\quad 0.365918 \mathrm{~Hz}$

AQ $\quad 1.3664756 \mathrm{sec}$

$36 \quad 574.7$

DN $\quad 41.700$ usec

JE $\quad 6.00$ usec

TE $\quad 298.8 \mathrm{~K}$

J1 $2.00000000 \mathrm{sec}$

YCREST $\quad 0.00000000 \mathrm{sec}$

YCWRK $\quad 0.01500000 \mathrm{sec}$

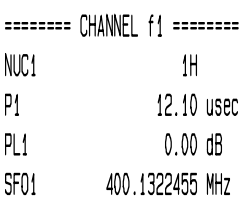

F2 - Processing parameters

SI $\quad 32768$

SF $\quad 400.1300097 \mathrm{NHz}$

NON EM

$558 \quad 0$

LB $\quad 0.00 \mathrm{~Hz}$

$G B \quad 0$

$\begin{array}{ll}x & 0.10\end{array}$
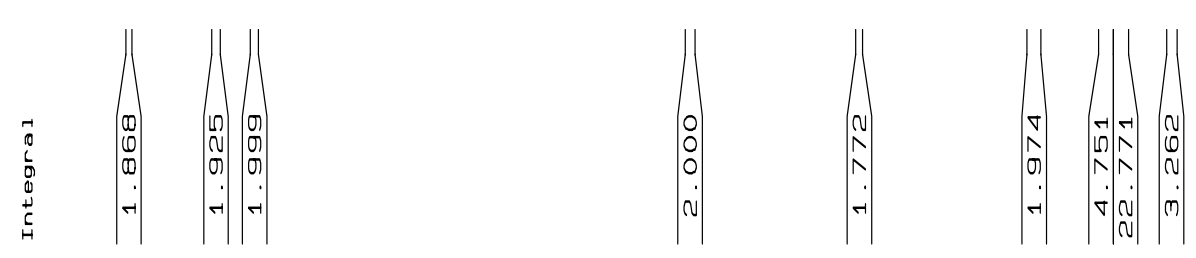

$\begin{array}{lc}10 \text { NMR plot parameters } \\ C X & 22.00 \mathrm{~cm} \\ C Y & 10.00 \mathrm{~cm} \\ F-1 P & 9.000 \mathrm{ppm} \\ -1 & 3601.17 \mathrm{~Hz} \\ F 2 \mathrm{P} & -0.500 \mathrm{ppm} \\ F 2 & -200.07 \mathrm{~Hz} \\ \text { PPMCM } & 0.43182 \mathrm{ppm} / \mathrm{cm} \\ \text { HZCM } & 172.78342 \mathrm{~Hz} / \mathrm{cm}\end{array}$

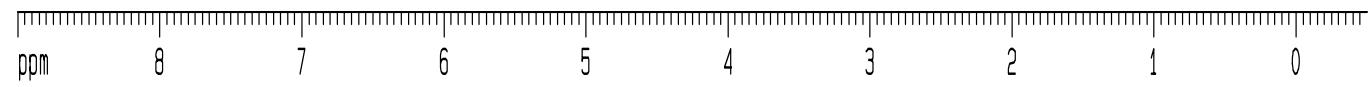

Figure S19: ${ }^{1} \mathrm{H}$ NMR spectra of $\mathbf{6 b}$ 


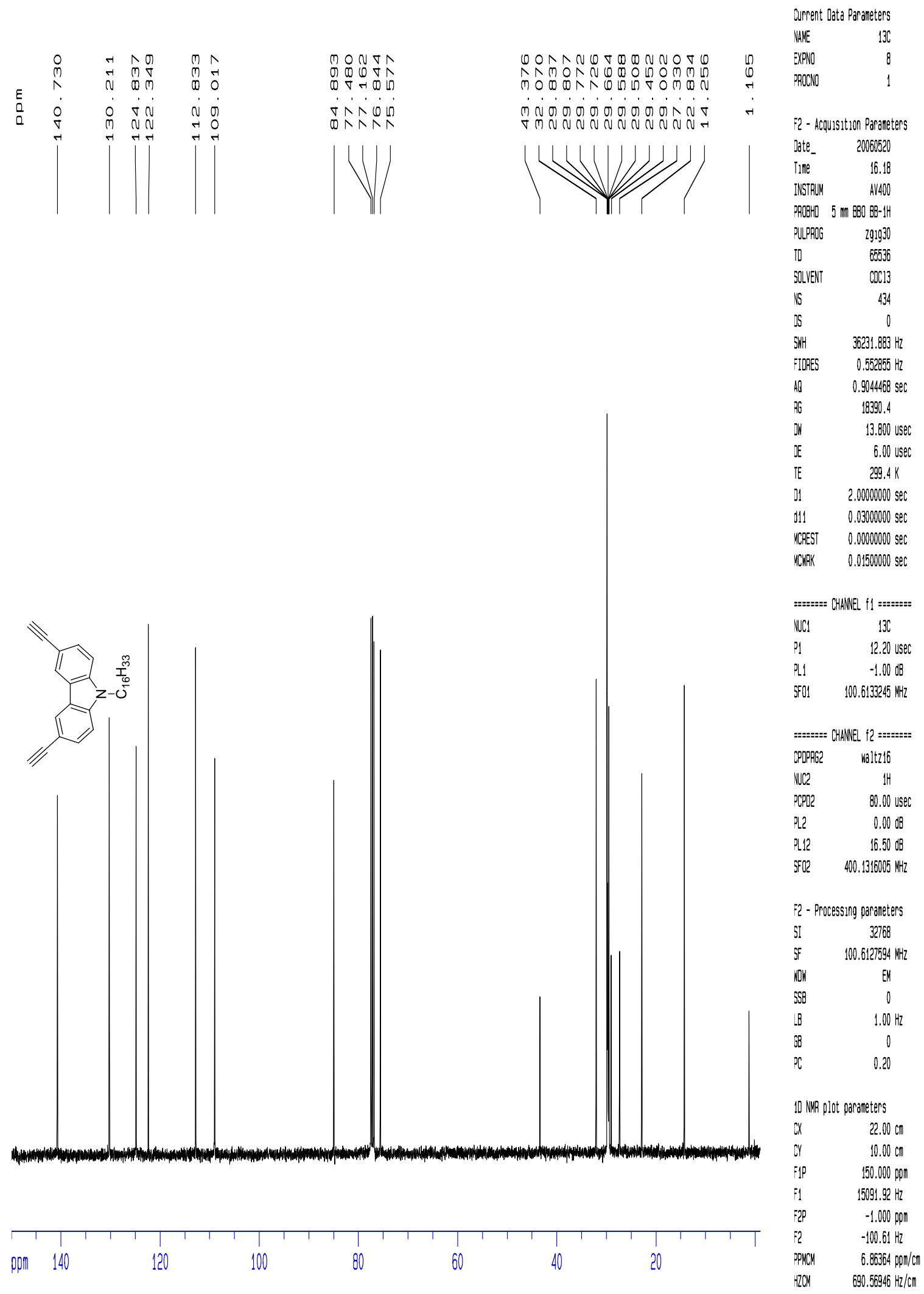

Figure S20: ${ }^{13} \mathrm{C}$ NMR spectra of $\mathbf{6 b}$ 


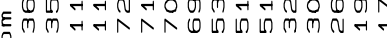

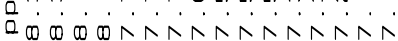

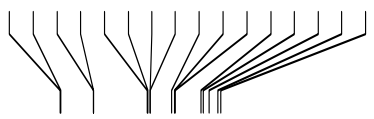

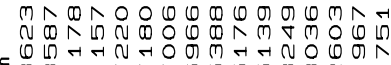

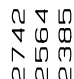

$\Delta \nabla \dot{\nabla}$

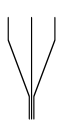

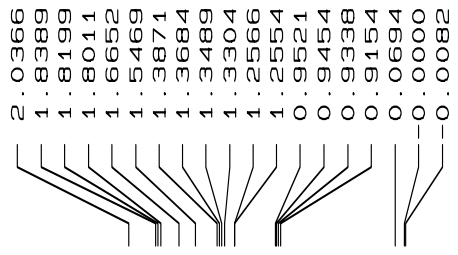

Surrent Data Parameters

VAME $\quad$ IH

JROCNO

:2 - Accuisition Parameters

Jate_ 20050923

Time $\quad 14.06$

INSTRUM AVAOO

गPOBHO $5 \mathrm{~m}$ ח BBO B8-1H

JULPPOG 2930

TD 32768

SOLVENT COC13

$\begin{array}{lr}15 & 16 \\ 35 & 0\end{array}$

5以H $\quad 8012.820 \mathrm{~Hz}$

:IDDES $\quad 0.244532 \mathrm{~Hz}$

AQ $\quad 2.0447731 \mathrm{sec}$

$36 \quad 1149.4$

JW $\quad 62.400$ usec

JE $\quad 6.00$ usec

TE $\quad 298.0 \mathrm{~K}$

J1 $2.00000000 \mathrm{sec}$

YCREST $\quad 0.00000000 \mathrm{sec}$

VCWHK $\quad 0.01500000 \mathrm{sec}$

:=z=:=: CHAWEL $\uparrow 1$ =:=:=:=

VUC1 $\quad$ H

ग1 8.00 usec

ILI $-4.00 \mathrm{~dB}$

3F01 400.1327722 MHz

:2 - Processing paraneters

3I 32768

3F $\quad 400.1300093 \mathrm{NHz}$

HOH EM

SSB -0.00

B $\quad 0.00 \mathrm{~Hz}$

$\begin{array}{lr}3 B & 0 \\ x & 0.10\end{array}$

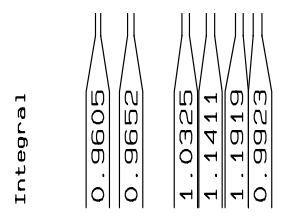

$\left|\begin{array}{l}\mid \\ 0 \\ m \\ 0 \\ 7 \\ \vdots \\ 0\end{array}\right|$

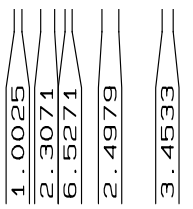

10 NMR plot paraneters

DX $\quad 22.00 \mathrm{~cm}$

cy $\quad 10.00 \mathrm{~cm}$

$=1 \mathrm{p} \quad 9.000 \mathrm{ppm}$

$=1 \quad 3601.17 \mathrm{~Hz}$

$=2 \mathrm{co} \quad-0.500 \mathrm{ppm}$

$=2 \quad-200.07 \mathrm{~Hz}$

JPNCM $\quad 0.43182 \mathrm{pon} / \mathrm{cm}$

tZCM $\quad 172.78342 \mathrm{~Hz} / \mathrm{cm}$

Figure S21: ${ }^{1} \mathrm{H}$ NMR spectra of 7a 


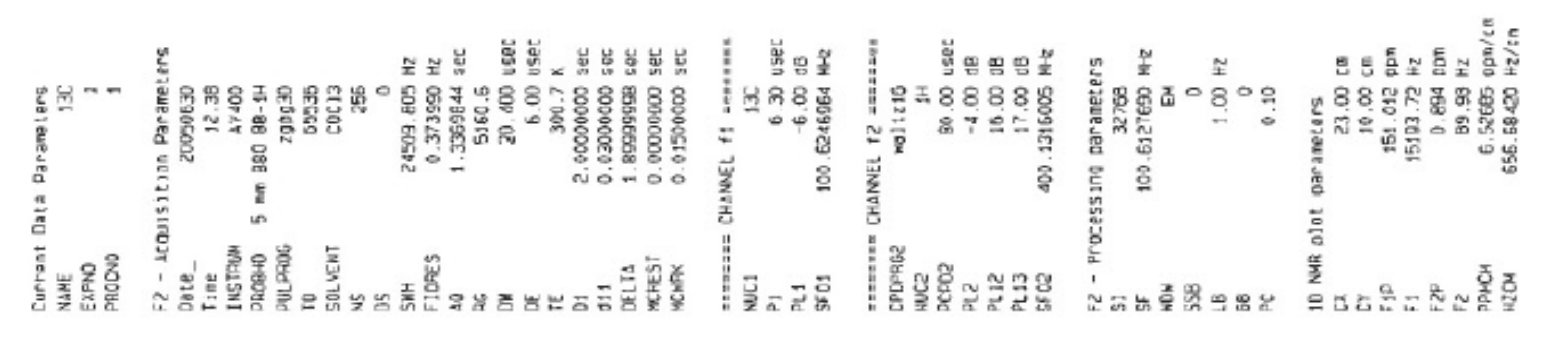

$\angle C 8 \varepsilon \square$

19,02

$\checkmark 96^{\circ} \propto 6$

CG: $2 \mathrm{C}$

만

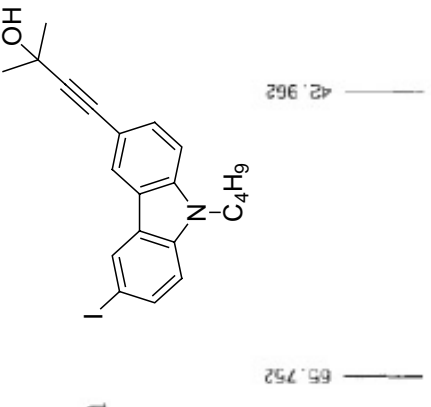

$\pi$

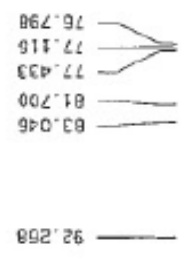

892' $26-$

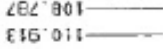

DIE हा-

DEE $121-$

$26 \mathrm{C}^{\circ} \rightarrow 21$

8962.62! $62 !$

EQG'PE S-

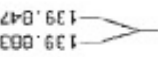

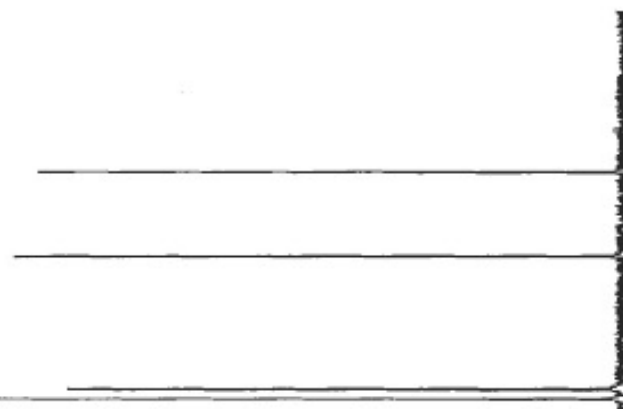

E

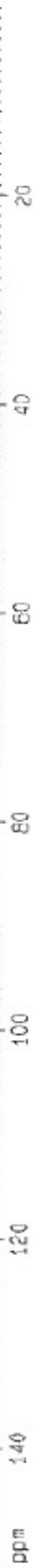

Figure S22: ${ }^{13} \mathrm{C}$ NMR spectra of 7a 

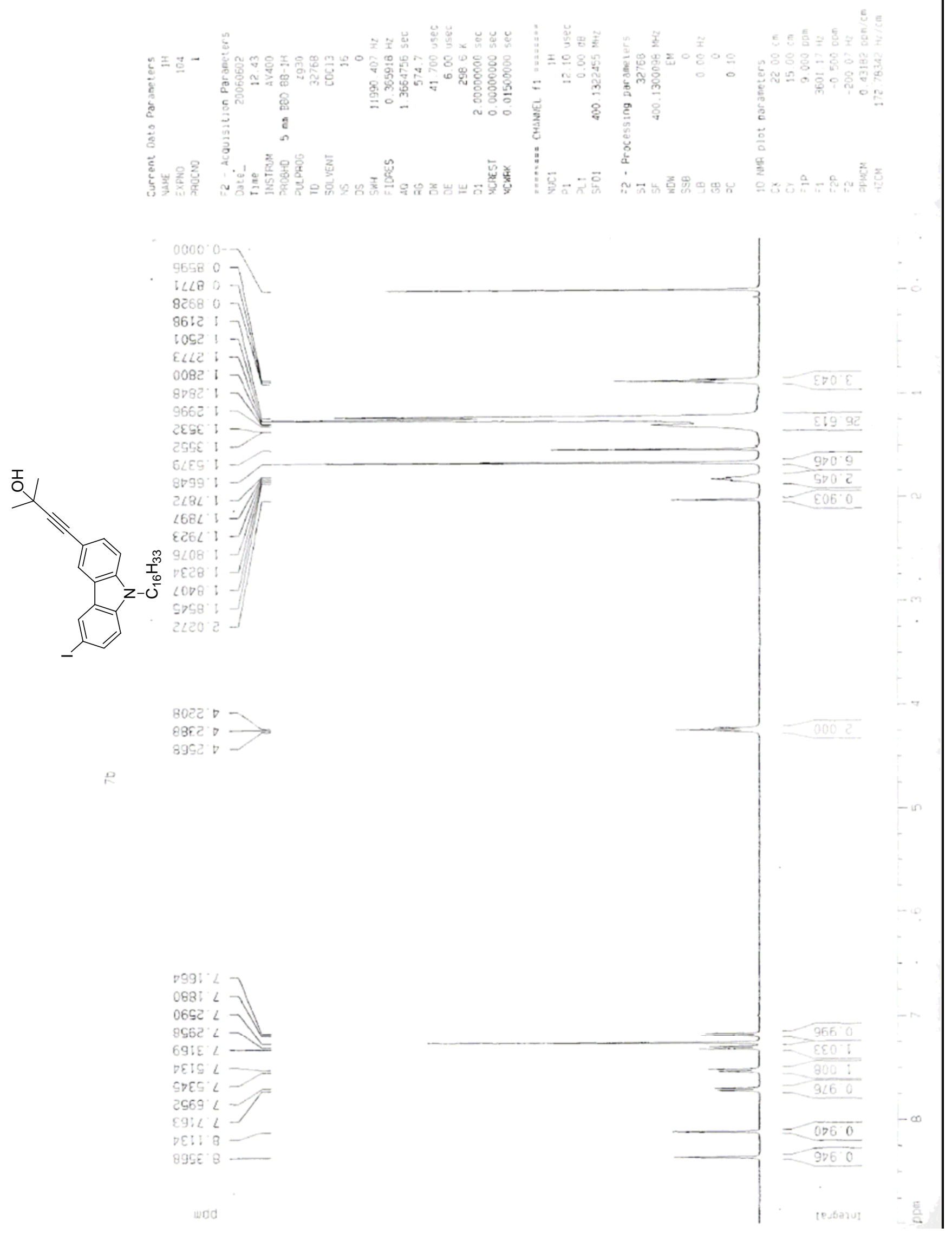

Figure S23: ${ }^{1} \mathrm{H}$ NMR spectra of $\mathbf{7 b}$ 

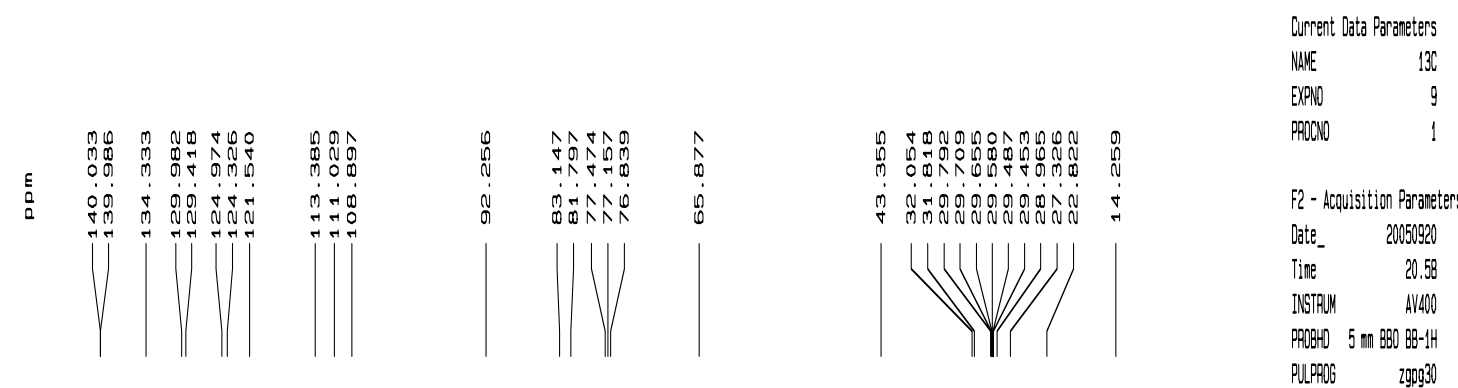

$\because 2$ - Acquisition Paraneters

Jate_ 20050920

Time 20.58

INSTRUM AM4OO

PPDBCD 5 m BBD BB-1H

PULPROG 2gDg30

I0 $\quad 65536$

SOLVENT COCI3

NS 762

$\begin{array}{lc}\text { JS } & 0 \\ \text { SWH } & 21509805 \mathrm{~Hz}\end{array}$

FIDFES $\quad 0.373990 \mathrm{~Hz}$

Ad $\quad 1.3369844 \mathrm{sec}$

16 $\quad 32768$

J) 20.400 usec

JE $\quad 6.00$ usec

TE $298.0 \mathrm{~K}$

D! $2.00000000 \mathrm{sec}$

$0.03000000 \mathrm{sec}$

JELLTA $1.89999998 \mathrm{sec}$

YCREST $0.00000000 \mathrm{sec}$

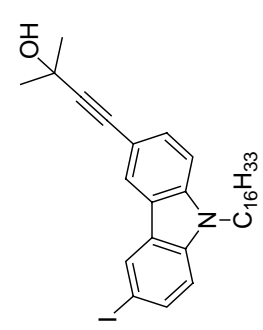

MCWRK $\quad 0.01500000 \mathrm{sec}$

=:=:=:= CHANEL f1 =:=:-:=

NUC1 $13 C$

916.30 usec

DL1 $\quad-6.00 \mathrm{~dB}$

SFO1 100.6246964 MHz

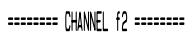

IPDPPG? Naltz16

NUC2 $\quad$ H

DCPD2 $\quad 80.00$ USEC

D.L? $-4.00 \mathrm{~dB}$

P.12 $\quad 16.00 \mathrm{~dB}$

$2.13 \quad 17.00 \mathrm{~dB}$

SFO2 400.1316005 MHz

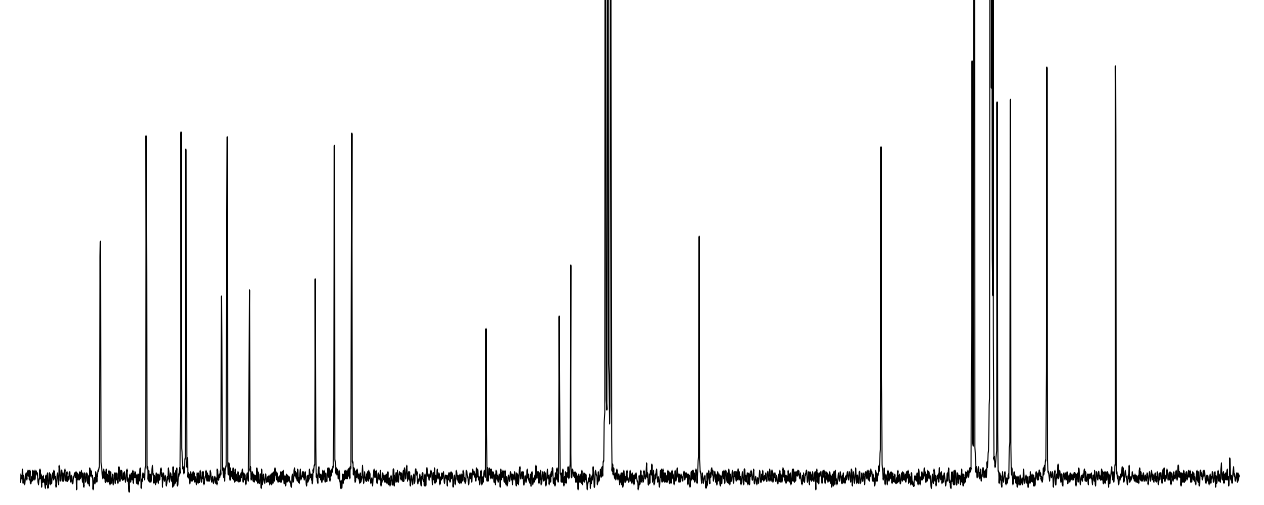

$\because 2$ - Processing parameters

SI 32768

If $\quad 100.6127586 \mathrm{MH}$

NOK EN

SSB

B $\quad 3.00 \mathrm{~Hz}$

$\begin{array}{lr}38 & 0 \\ x & 0.10\end{array}$

10 NWh plot parameters

cx $\quad 23.00 \mathrm{~cm}$

Cy $\quad 10.00 \mathrm{~cm}$

$\quad 150.000$ pon

$\because 1 \quad 15091.91 \mathrm{~Hz}$

$-2 \mathrm{p} \quad-1.000 \mathrm{ppon}$

$\because 2 \quad-100.61 \mathrm{~Hz}$

PPMCY $\quad 6.56522$ pDM/cm

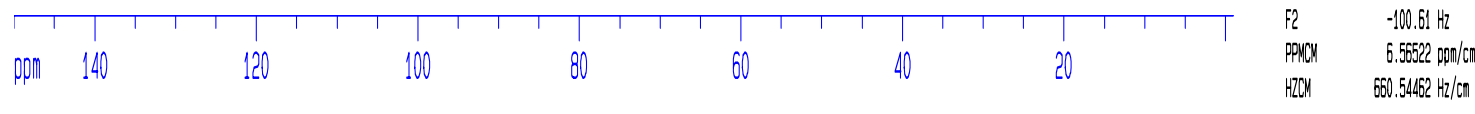

Figure S24: ${ }^{13} \mathrm{C}$ NMR spectra of $\mathbf{7 b}$ 

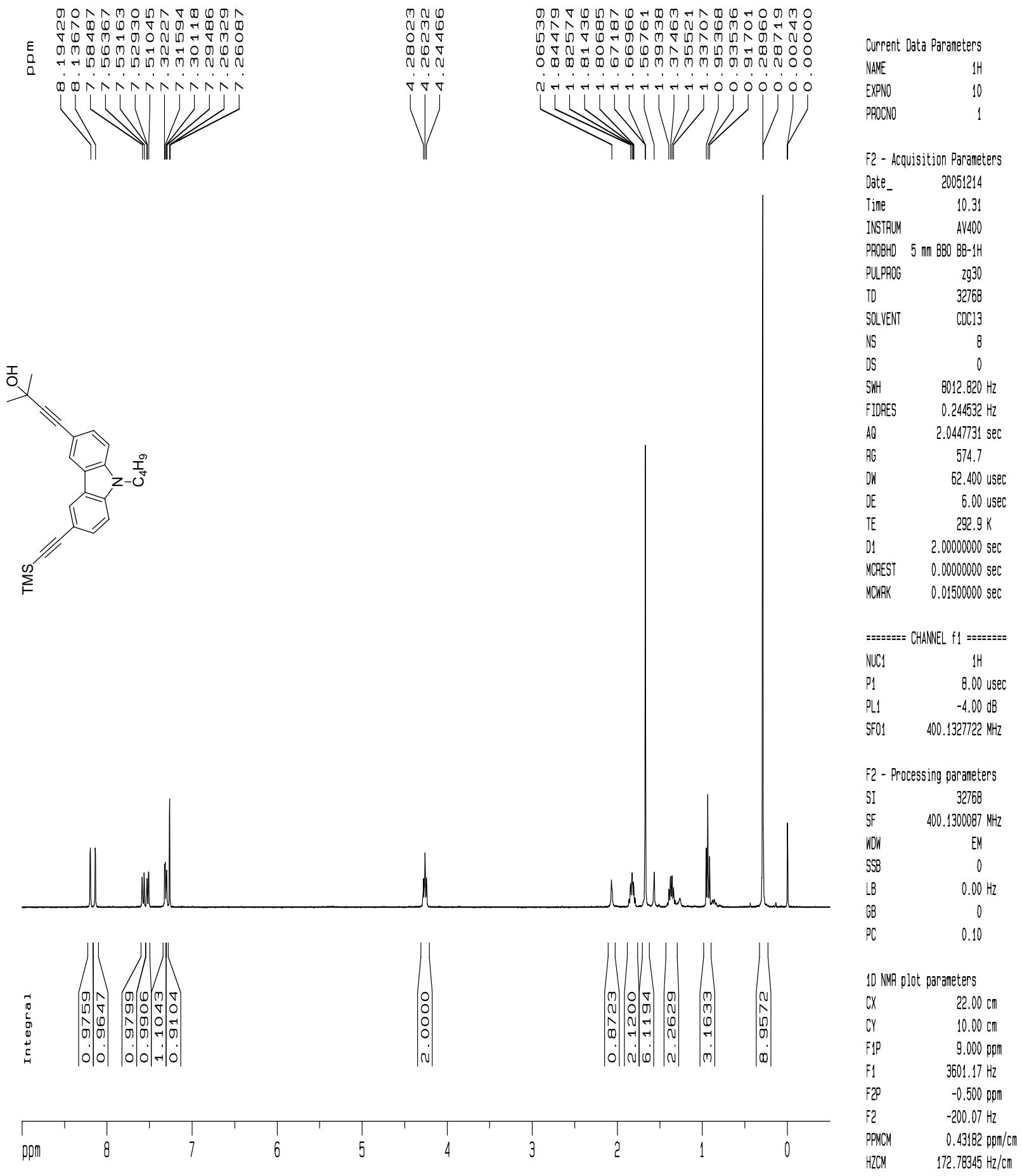

Figure S25: ${ }^{1} \mathrm{H}$ NMR spectra of 8 a 


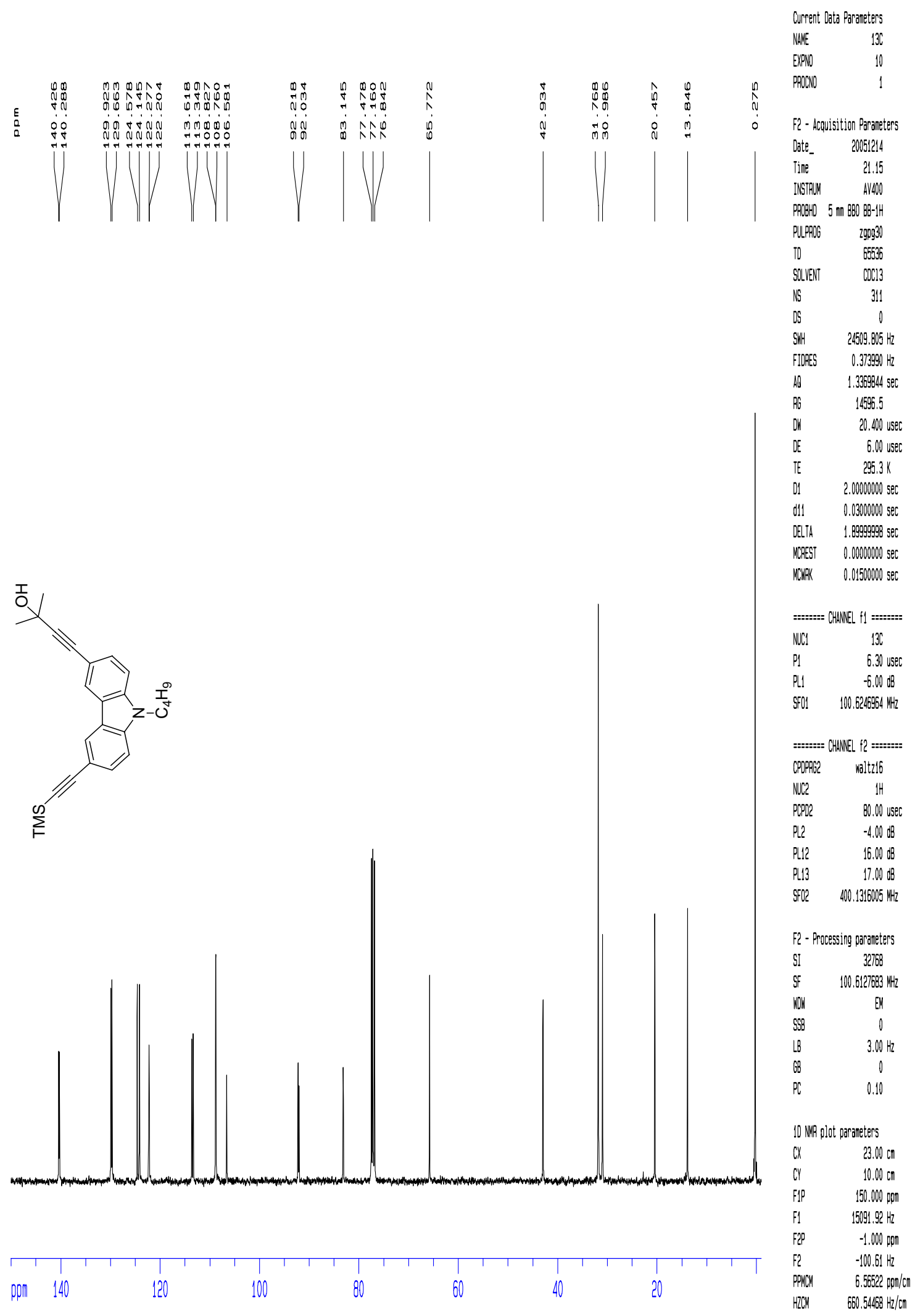

Figure S26: ${ }^{13} \mathrm{C}$ NMR spectra of $\mathbf{8 a}$ 

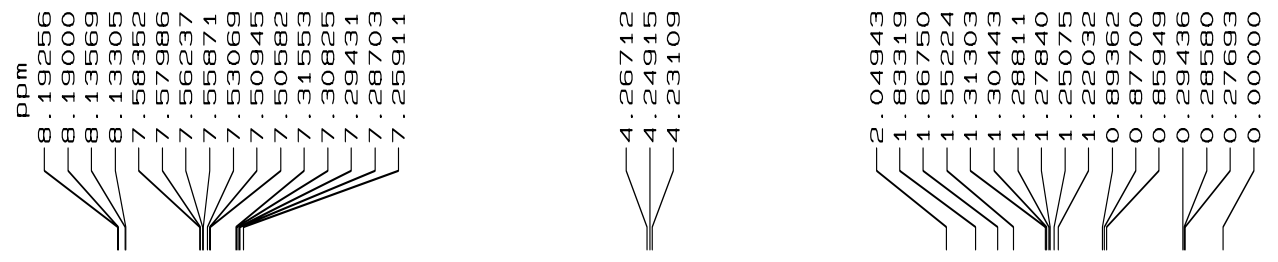

Current Data Parameters

VAIE

EXPNO

TROCNO 1

=2 - Acquisition Paraneters

Jate_ 20050923

Time $\quad 14.30$

INSTRUM AV40O

วROBHD 5 ח ח BBO BB-1H

गULPROG 2030

TO 32768

SOLENT COC13

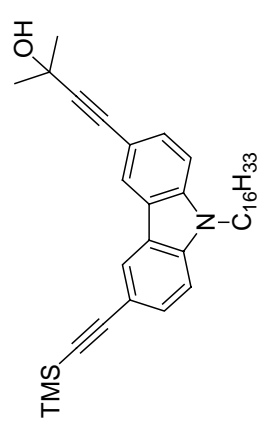

IS 10

SWH $8012.820 \mathrm{~Hz}$

=IDDES $\quad 0.244532 \mathrm{~Hz}$

AQ $2.0447731 \mathrm{sec}$

$36 \quad 406.4$

J11 $\quad 62.400$ usec

JE $\quad 6.00$ usec

TE $298.0 \mathrm{~K}$

$31 \quad 2.00000000 \mathrm{sec}$

YCAEST $\quad 0.000000000 \mathrm{sec}$

YCWRK $\quad 0.01500000 \mathrm{sec}$

:zz:z:=s CHAWEL $f 1$ =z:z:z=s

VUC1 $\quad$ IH

ग1 8.00 usec

J.1 $-4.00 \mathrm{~dB}$

SF01 400.1327722 $\mathrm{NHz}$

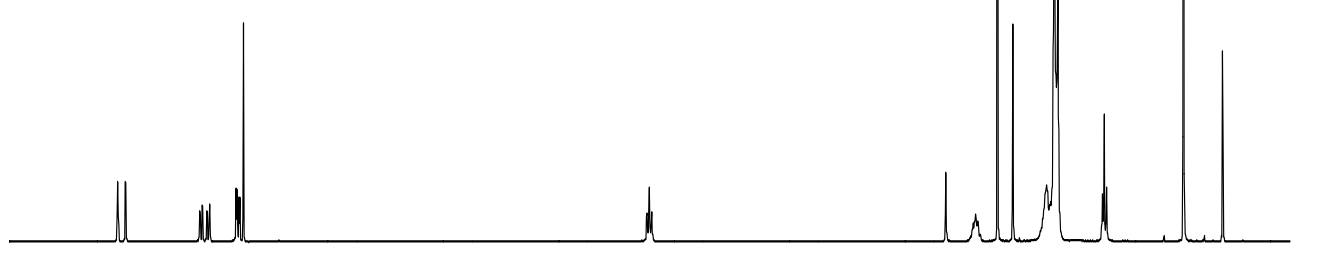

:2 - Processing parameters

SI $\quad 32760$

SF $\quad 400.1300098 \mathrm{NHz}$

HON EM

SSB

B $\quad 0.00 \mathrm{~Hz}$

$\begin{array}{lr}3 B & 0 \\ X & 0.10\end{array}$
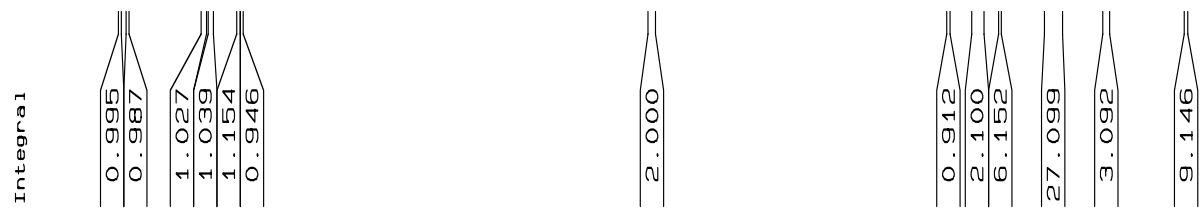

10 NWR plot paraneters

ox $\quad 22.00 \mathrm{~cm}$

CY $\quad 10.00 \mathrm{~cm}$

$=1 \mathrm{p} \quad 9.000 \mathrm{ppm}$

$3601.17 \mathrm{~Hz}$

$-0.500 \mathrm{ppm}$

$-200.07 \mathrm{~Hz}$

$0.43182 \mathrm{ppm} / \mathrm{cm}$

Figure S27: ${ }^{1} \mathrm{H}$ NMR spectra of $\mathbf{8 b}$ 

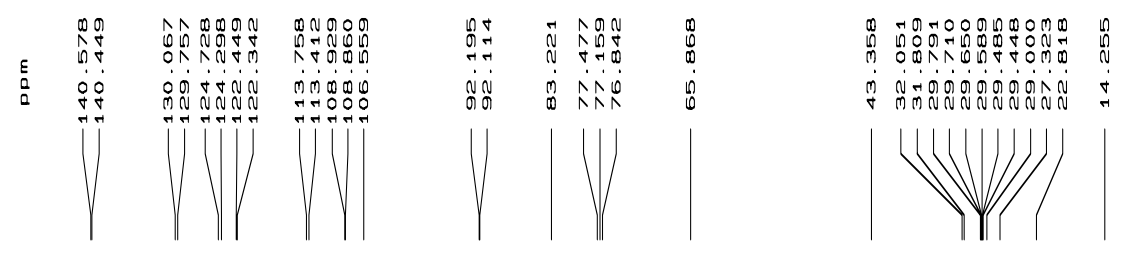

Wurrent Data Paraneters

:XPNO

$\because ?$ - Acquisition Parameters

Jate_ 20050920

Time $\quad 19.41$

INSTRUM AV4OO

JPOBHO 5 ח ח BBO BB-1H

गULPDOG 2909930

To $\quad 65356$

3OLVENT COCL3

$15 \quad 256$

5WH $24509.805 \mathrm{~Hz}$

IIDRES $\quad 0.373990 \mathrm{~Hz}$

Al $\quad 1.3369844 \mathrm{sec}$

36 5160.6

J1 20.400 useC

Je $\quad 6.00$ usec

IE $297.3 \mathrm{~K}$

J1 $2.00000000 \mathrm{sec}$

J11 $\quad 0.03000000 \mathrm{sec}$

JELTA 1.8999g9g8 sec

VCAEST $\quad 0.00000000 \mathrm{sec}$

YCWRK $0.01500000 \mathrm{sec}$

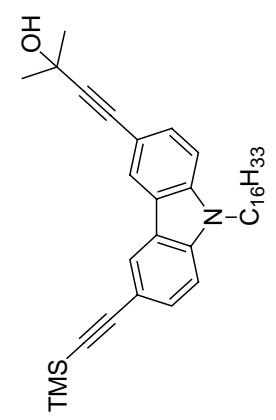

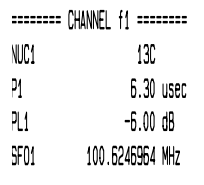

:=::=:: CHANEL $\uparrow$ : =::=::=

sPDPAG2 Naltzi6

$\begin{array}{ll}\text { WCE } & \text { IH } \\ \text { उCPD? } & \text { BD }\end{array}$

ग.? $\quad-4.00 \mathrm{~dB}$

$21.12 \quad 16.00 \mathrm{~dB}$

$2013 \quad 17.00 \mathrm{~dB}$

3502 $400.1316005 \mathrm{MHz}$

$: 2$ - Processing parameters

3I 32768

3f $\quad 100.6127594 \mathrm{MHz}$

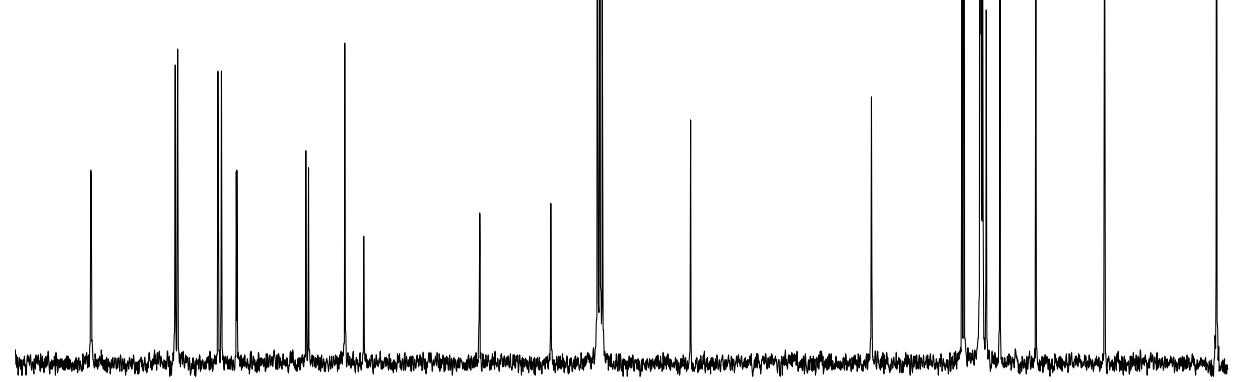

NOW

B $\quad 3.00 \mathrm{~Hz}$

$3 B \quad 0$

$x \quad 0.10$

10 NHA plot parameters

ox $\quad 23.00 \mathrm{~cm}$

iy $\quad 10.00 \mathrm{~cm}$

$=19 \quad 150.000$ ppo

$=15091.91 \mathrm{~Hz}$

$=2 p \quad-1.000$ ppm

$=2 \quad-100.61 \mathrm{~Hz}$

गPHCH $\quad 6.56522 \mathrm{pDm} / \mathrm{cm}$

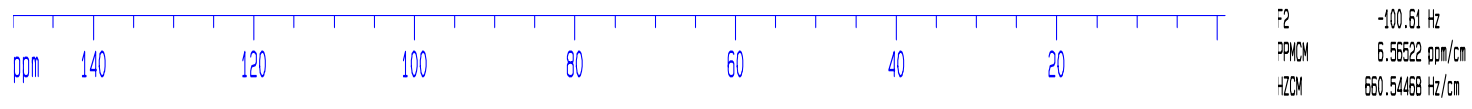

Figure S28: ${ }^{13} \mathrm{C}$ NMR spectra of $\mathbf{8 b}$ 


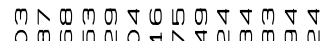

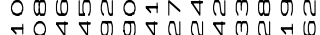
ह ก $\mathrm{N}$ ส

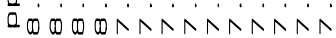
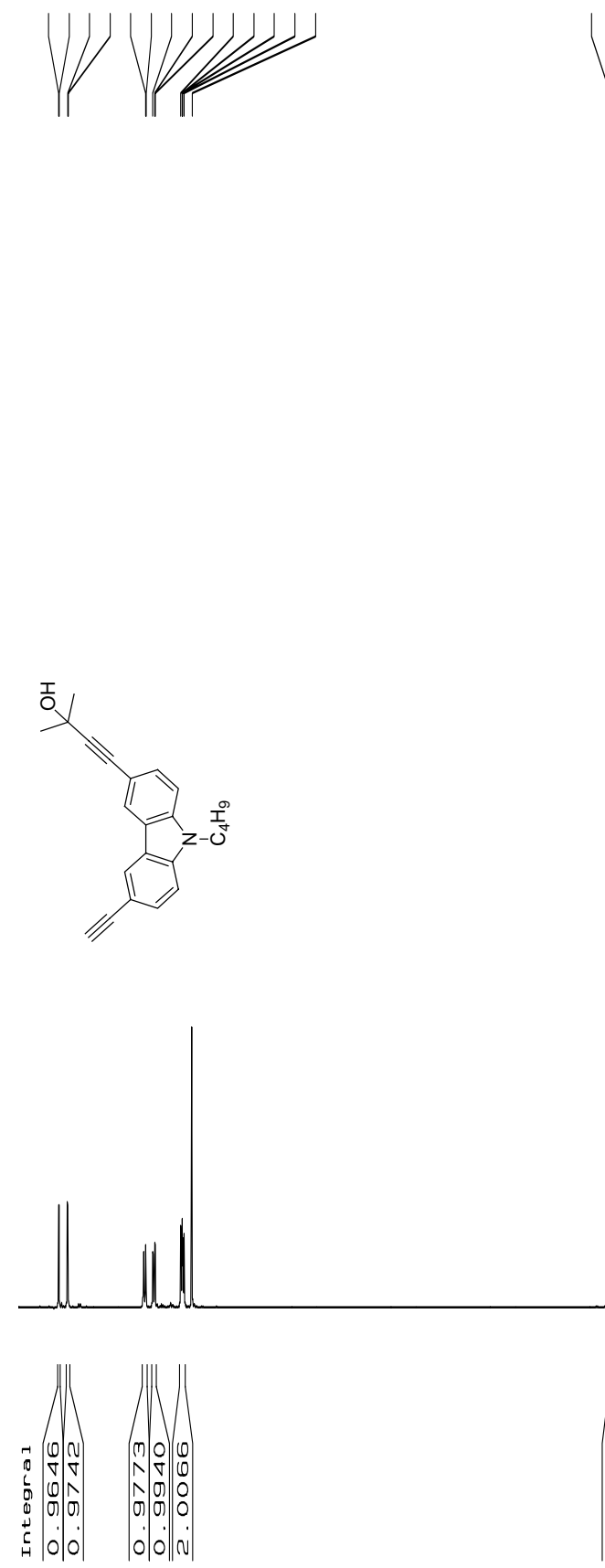

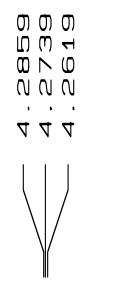

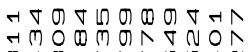
a mONMUN

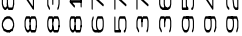
तनन4न4नHOO०
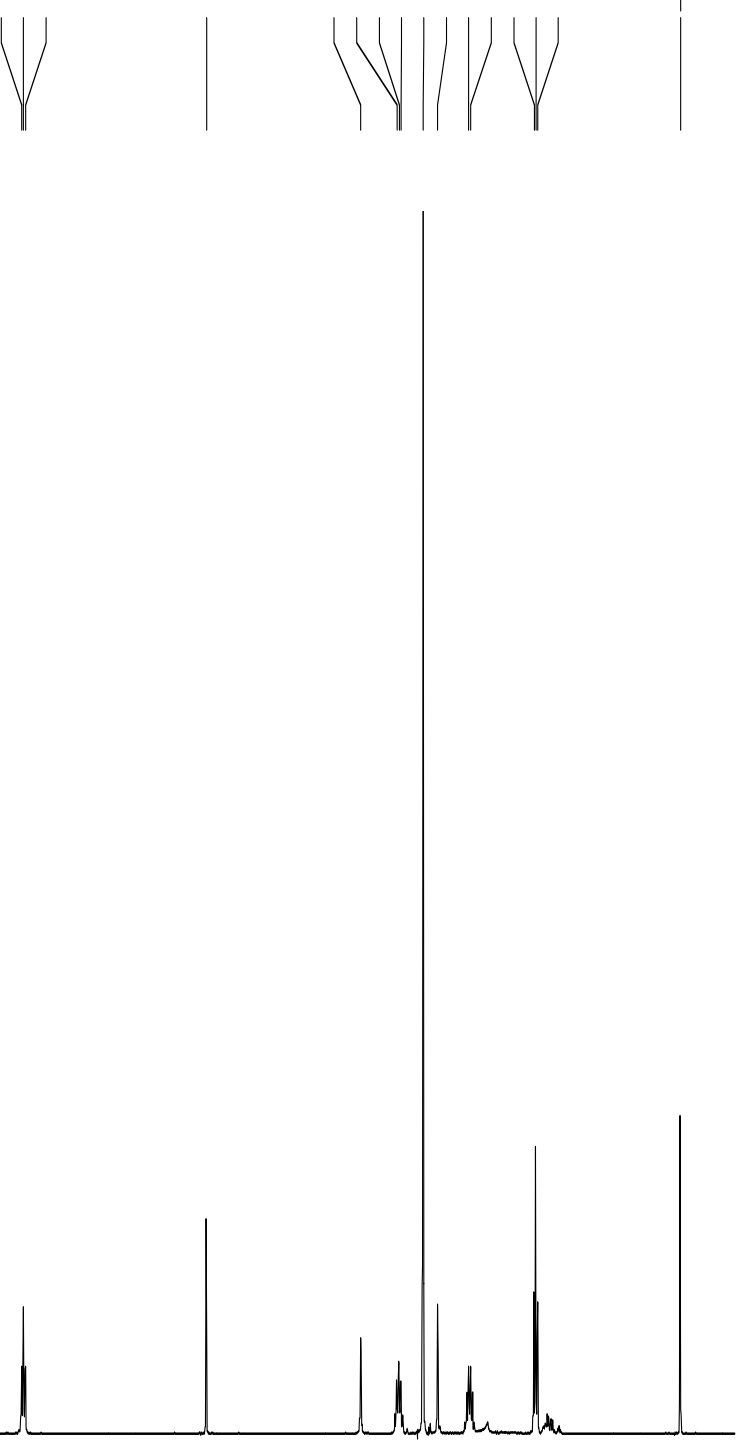

\begin{tabular}{|c|c|}
\hline NUC1 & $1 H$ \\
\hline 71 & 10.50 usec \\
\hline PLI & $1.30 \mathrm{~dB}$ \\
\hline SFO1 & $600.1324671 \mathrm{NHz}$ \\
\hline \multicolumn{2}{|c|}{ :२ - Processing paraneters } \\
\hline SI & 32768 \\
\hline SF & $600.1300088 \mathrm{NHz}$ \\
\hline NON & EM \\
\hline SSB & 0 \\
\hline B & $0.00 \mathrm{~Hz}$ \\
\hline BB & 0 \\
\hline x & 1.00 \\
\hline \multicolumn{2}{|c|}{10 NMR plot paraneters } \\
\hline dX & $24.99 \mathrm{~cm}$ \\
\hline ¿Y & $10.00 \mathrm{~cm}$ \\
\hline :1p & $8.500 \mathrm{ppm}$ \\
\hline-1 & $5101.10 \mathrm{~Hz}$ \\
\hline Г२p & $-0.500 \mathrm{ppn}$ \\
\hline$\because 2$ & $-300.07 \mathrm{~Hz}$ \\
\hline PPYCY & $0.36019 \mathrm{ppm} / \mathrm{c}$ \\
\hline HZCN & $216.15794 \mathrm{~Hz} / \mathrm{cm}$ \\
\hline
\end{tabular}



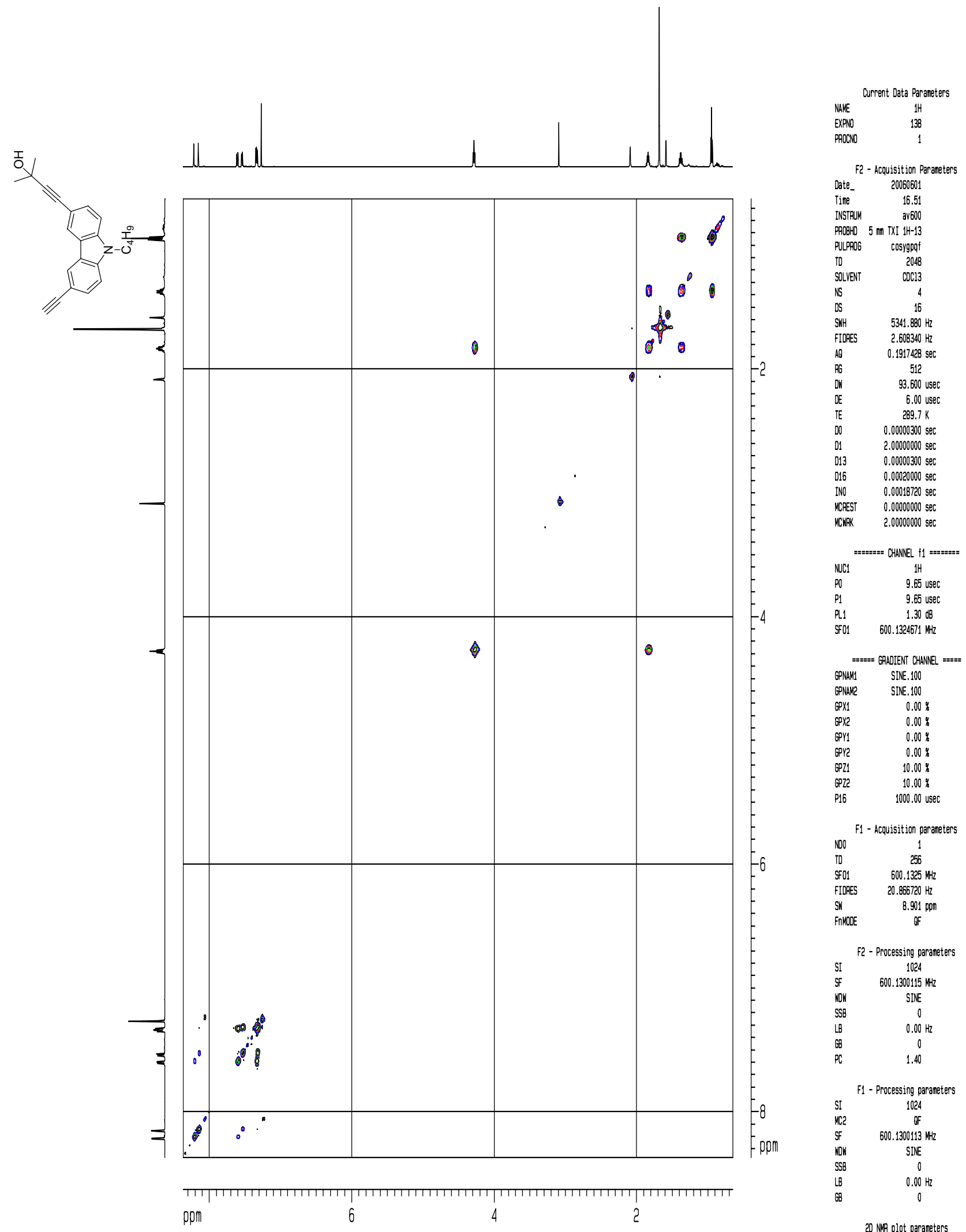

$\begin{array}{lc} & \text { F1 - Acquisition paraneters } \\ \text { NDO } & 1 \\ \text { TD } & 256 \\ \text { SF01 } & 600.1325 \mathrm{HHz} \\ \text { FIDRES } & 20.866720 \mathrm{~Hz} \\ \text { SW } & 8.901 \text { pPח } \\ \text { FOMOOE } & \text { OF }\end{array}$

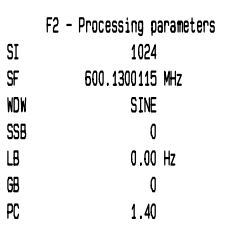

$\begin{array}{lc} & \text { F1 - Processing paraneters } \\ \text { SI } & 1024 \\ \text { AC2 } & \text { OF } \\ \text { SF } & 600.1300113 \mathrm{HHz} \\ \text { NOW } & \text { SINE } \\ \text { SSB } & 0 \\ \text { GB } & 0.00 \mathrm{~Hz} \\ \text { GB } & 0\end{array}$

20 NNR plot parameters

cx2 $15.00 \mathrm{~cm}$

Figure S30: COSY spectra of 9a 


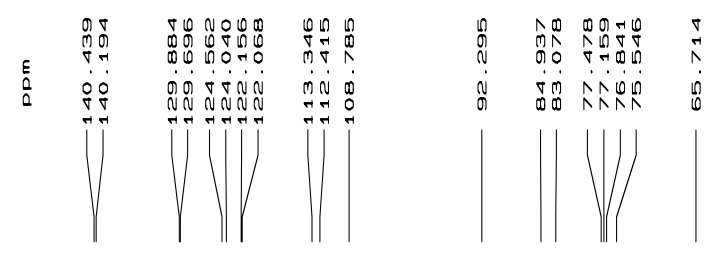

Current Data Peraneters
NAME
EXPNO

PAOCNO 1

F2 - Acquisition Parameters

Date_ 20051214

Tine 21.4

INSTRUM AVMOO

PAOBHO 5 ח $\mathrm{BBO}$ BB-1H

PULPPOG 2909030

TD $\quad 65536$

SOLVENT COC13

NS 30

DS $\quad 0$

SWH $\quad 24509.805 \mathrm{~Hz}$

FIDRES $\quad 0.373990 \mathrm{~Hz}$

Ad $\quad 1.3369844 \mathrm{sec}$

PG 8192

DN $\quad 20.400$ usec

DE $\quad 6.00$ usec

TE $\quad 205.5 \mathrm{~K}$

DI $2.00000000 \mathrm{sec}$

d11 $\quad 0.03000000 \mathrm{sec}$

DELTA $\quad 1.89999998 \mathrm{sec}$

MCREST $\quad 0.00000000 \mathrm{SeC}$

MCRRK $\quad 0.01500000 \mathrm{sec}$

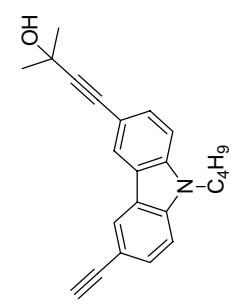

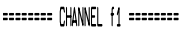

\begin{tabular}{|c|c|}
\hline NCI & 136 \\
\hline$P 1$ & $6.30 \mathrm{usec}$ \\
\hline Pl! & $-6.00 \mathrm{~dB}$ \\
\hline SFO! & $100.62469564 \mathrm{MHz}$ \\
\hline \multicolumn{2}{|c|}{ 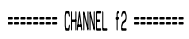 } \\
\hline CPDPAGQ & Maltzí6 \\
\hline NUC? & IH \\
\hline PCPO2 & 80.00 usec \\
\hline Pl? & $-4,00 \mathrm{~dB}$ \\
\hline PL12 & $16.00 \mathrm{~dB}$ \\
\hline PL13 & $17,00 \mathrm{~dB}$ \\
\hline $8=02$ & $400.1316005 \mathrm{WHz}$ \\
\hline
\end{tabular}

F2 - Processing parameters

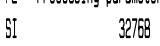

Sf $\quad 100.6127758 \mathrm{WHz}$

NON EN

$\begin{array}{lc}598 & 0 \\ L B & 3.00 \mathrm{~Hz}\end{array}$

bo $\quad 0$

PC $\quad 0.10$

10 NWR plot paraneters

cX $\quad 23.00 \mathrm{~cm}$

$\begin{array}{ll}\text { Cy } & 8.00 \mathrm{~cm}\end{array}$

Fip $\quad 150.000$ ppm

F1 $\quad 15091.92 \mathrm{~Hz}$

F20 $\quad-1.000$ ppm

F2 $\quad-100.61 \mathrm{~Hz}$

PPYCH $\quad 6.56522 \mathrm{pDm} / \mathrm{cm}$

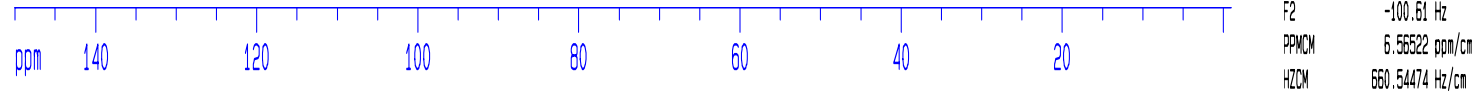

Figure S31: ${ }^{13} \mathrm{C}$ NMR spectra of $9 a$ 


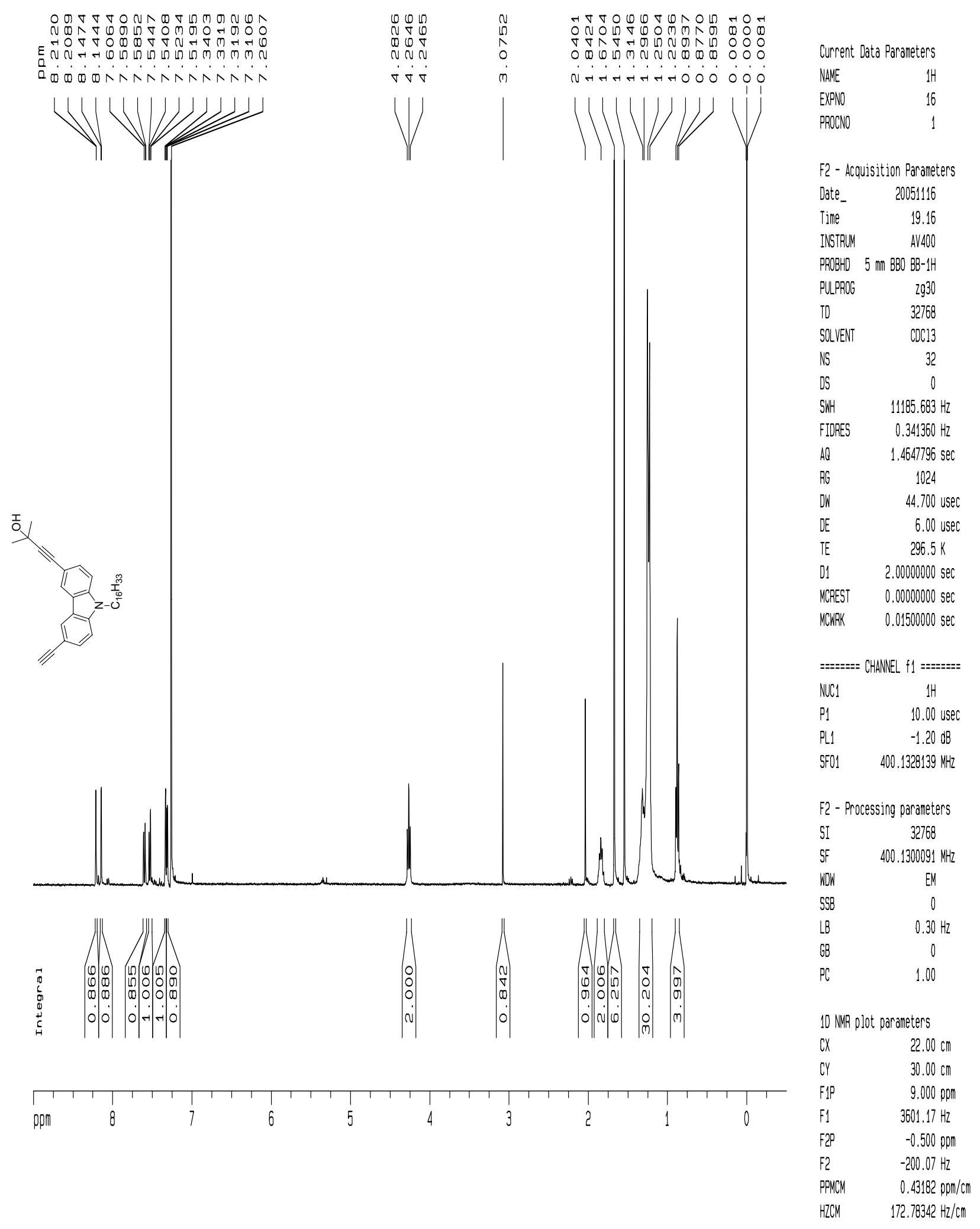

Figure S32: ${ }^{1} \mathrm{H}$ NMR spectra of $9 \mathbf{b}$ 

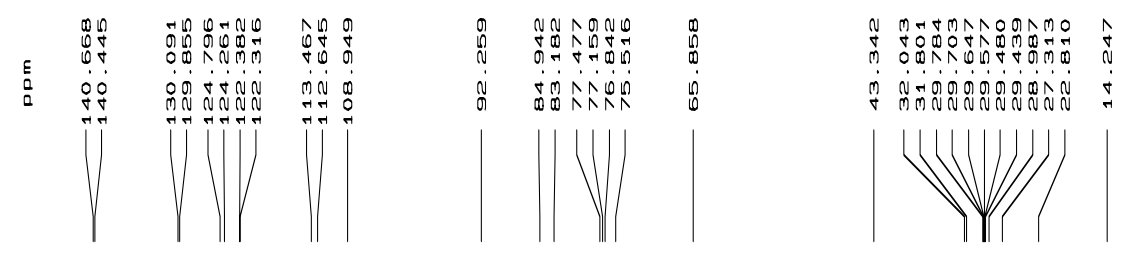

Cumpent Data Paraneters
WAFE
:XPON
PHOCNO

$\because 2$ - Acouisition Paraneters

Jate_ 20051117

Tine 21.07

INSTRUY A AMOO

YROOHO 5 I 18808 88-1H

गULPOOS 290930

TD 65536

SOLVENT COCI3

IS 851

SWH $24509.805 \mathrm{~Hz}$

-IDPES $\quad 0.373990 \mathrm{~Hz}$

40 $\quad 1.335984 \mathrm{sec}$

$76 \quad 1.556 .5$

3H 20.40 Usec

JE $\quad 6.00$ usec

TE $297.1 \mathrm{~K}$

$31 \quad 2.00000000 \mathrm{sec}$

$311 \quad 0.03000000$ sec

JELTA 1.89999989 SeC

YCEEST 0.00000000 sec

YCHAR $0.01500000 \mathrm{sec}$

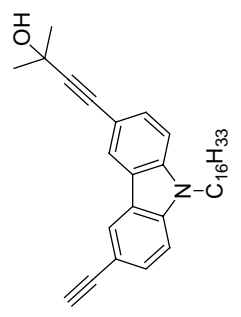

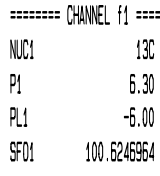

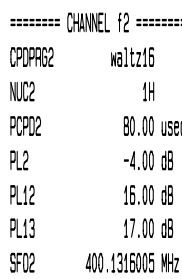

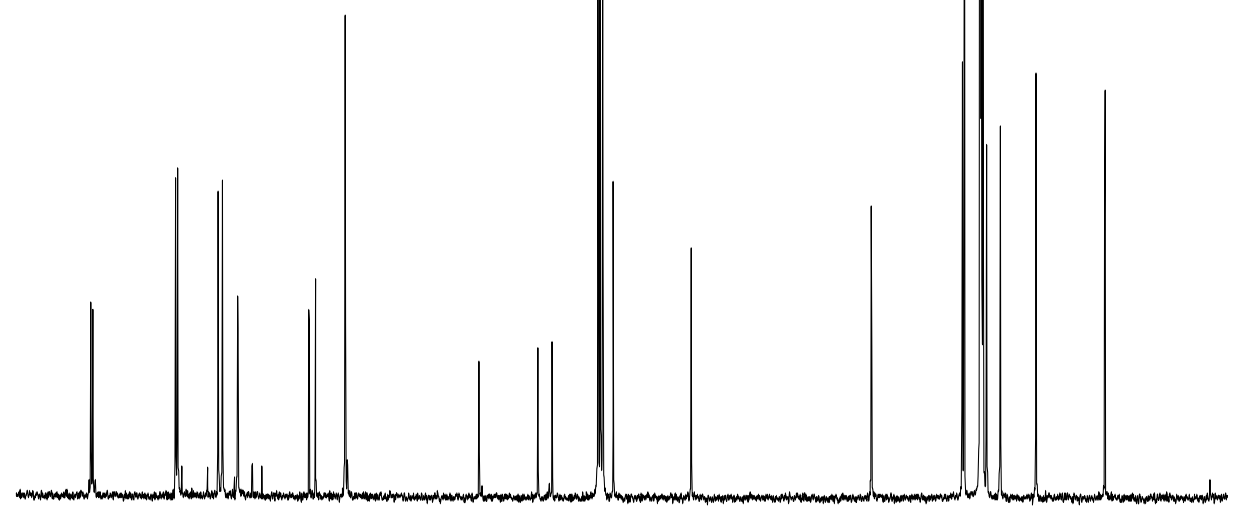

-2 - Processing paraneters

5I 32768

SF $\quad 100.6127609$ Whz

NOH EN

3980

B. $\quad 3.00 \mathrm{~Hz}$

$\begin{array}{ll}3 B & 0 \\ x & 0.10\end{array}$

10 WW plot paraneters

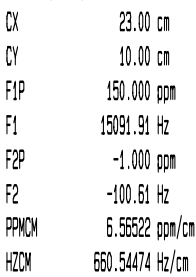

Figure S33: ${ }^{13} \mathrm{C}$ NMR spectra of $\mathbf{9 b}$ 

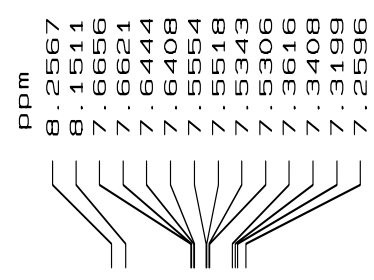
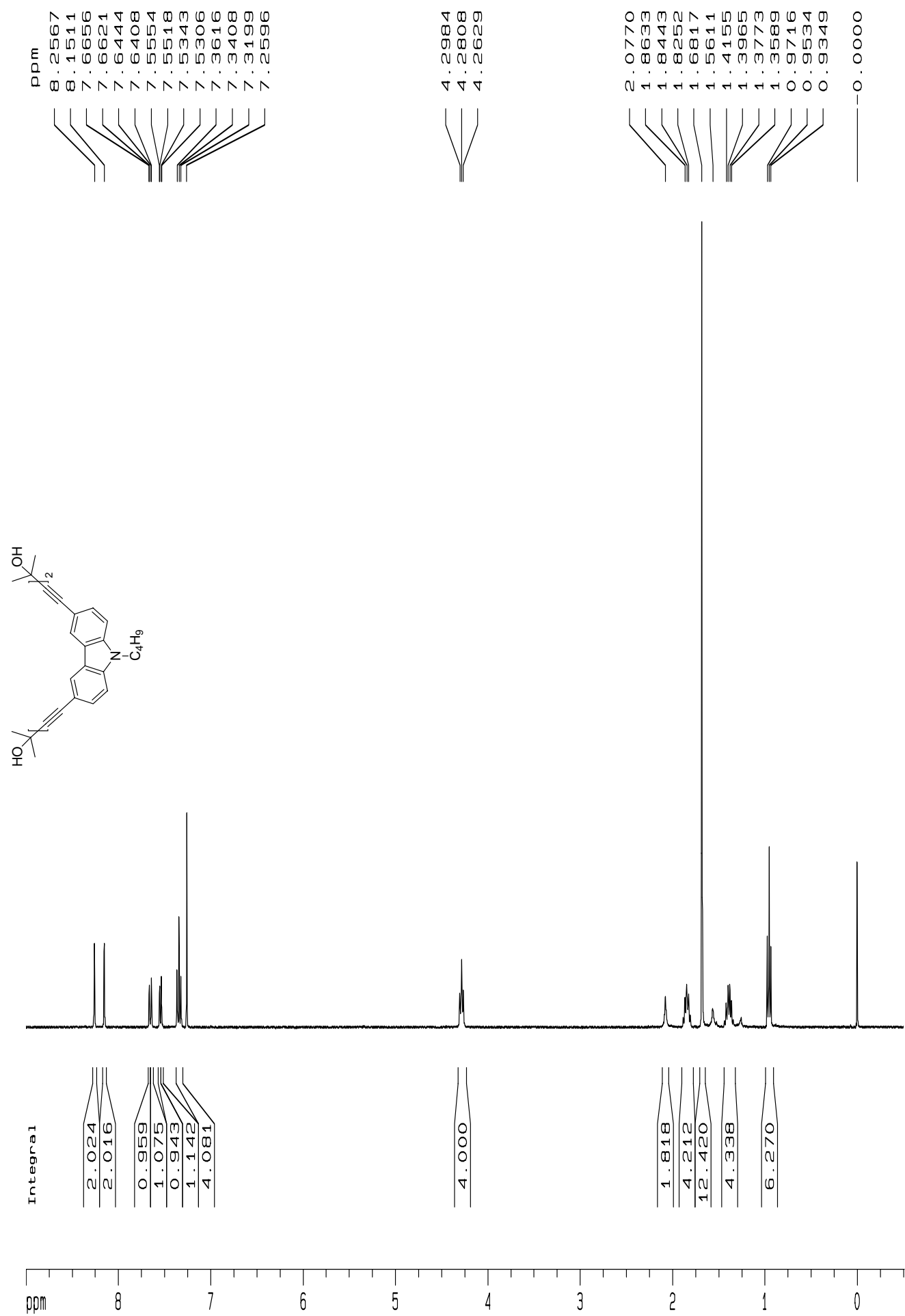
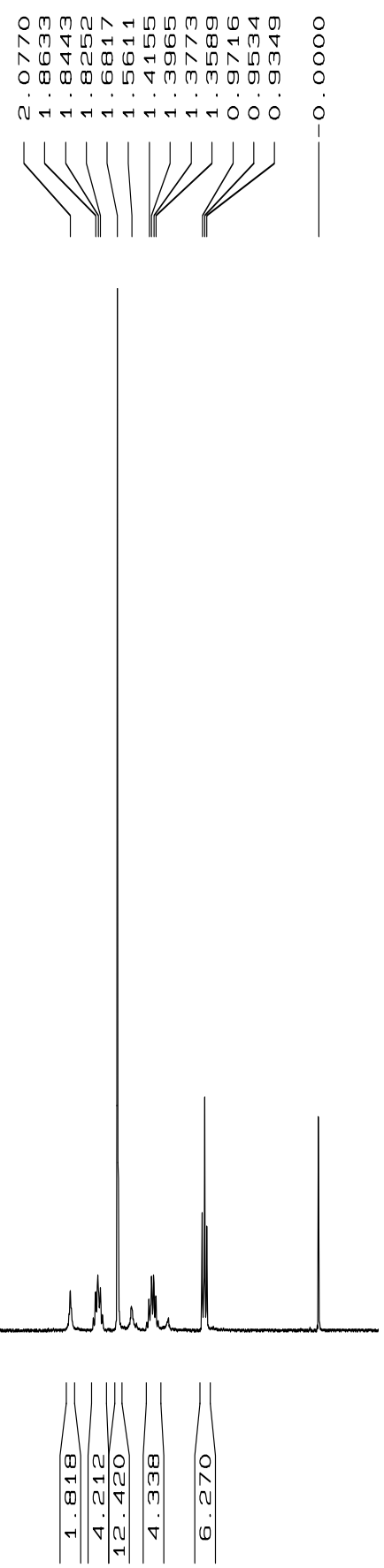

$\begin{array}{lr}\text { Current Data Parameters } \\ \text { NAME } & 1 \text { H } \\ \text { EXPNO } & 17 \\ \text { PROCNO } & 1\end{array}$

F2 - Acquisition Paraneters

Date_ 20051208

Time $\quad 17.34$

INSTRUM AV4OO

PROBHD 5 m $\mathrm{m}$ B8O B8-1H

PULPPOG 2930

TO 32768

SOLVENT COC13

NS 8

OS 0

5WH $\quad 8012.820 \mathrm{~Hz}$

FIDRES $\quad 0.24532 \mathrm{~Hz}$

AQ $\quad 2.047731 \mathrm{sec}$

RG $\quad 812.7$

DN $\quad 62.400$ usec

DE $\quad 6.00$ usec

TE $296.4 \mathrm{~K}$

D1 2.0000000 sec

MCREST $\quad 0.00000000 \mathrm{sec}$

MCWRK $\quad 0.01500000 \mathrm{sec}$

:=:=:=: CHAWEL $f 1$ =:z:=:=:

NUC1 $\quad$ H

P1 $\quad 10.00$ usec

PL1 $\quad-1.20 \mathrm{OB}$

SF01 400. $13332010 \mathrm{MHz}$

F2 - Processing paraneters

SI $\quad 32768$

SF $\quad 400.1300099 \mathrm{NHz}$

HOH EM

$598-0$

LB $\quad 0.00 \mathrm{~Hz}$

GB $\quad 0$

10 NWR plot parameters

\begin{tabular}{|c|c|}
\hline$x$ & $22.00 \mathrm{~cm}$ \\
\hline CY & $10.00 \mathrm{~cm}$ \\
\hline 1 P & 9.000 ppm \\
\hline & $36001.17 \mathrm{~Hz}$ \\
\hline$F 2 P$ & $-0.500 \mathrm{ppm}$ \\
\hline$F 2$ & $-200.07 \mathrm{~Hz}$ \\
\hline PNCM & $0.43182 \mathrm{pom}$ \\
\hline
\end{tabular}


Figure S34: ${ }^{1} \mathrm{H}$ NMR spectra of 10a

100

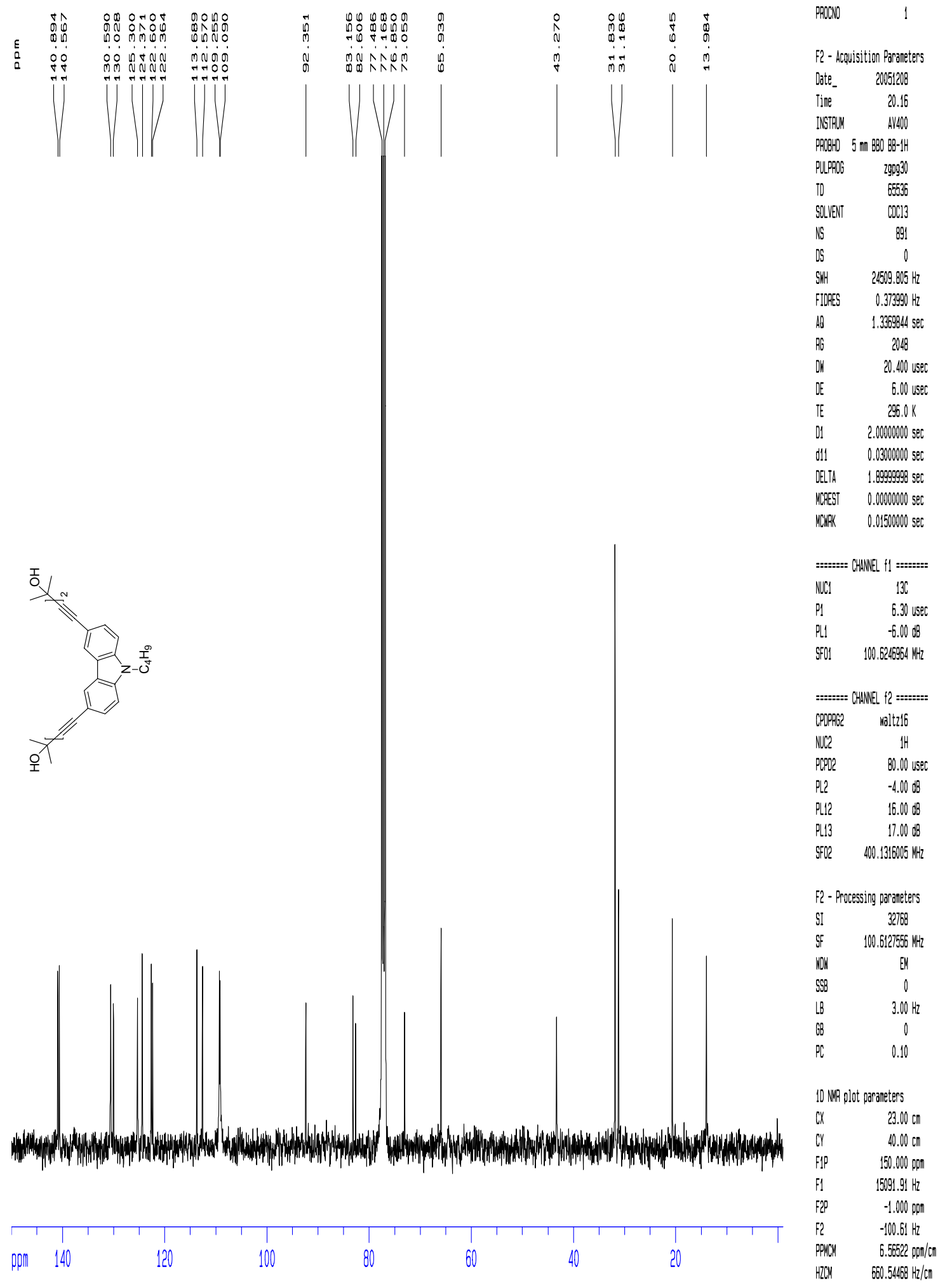


Figure S35: ${ }^{13} \mathrm{C}$ NMR spectra of 10a

100
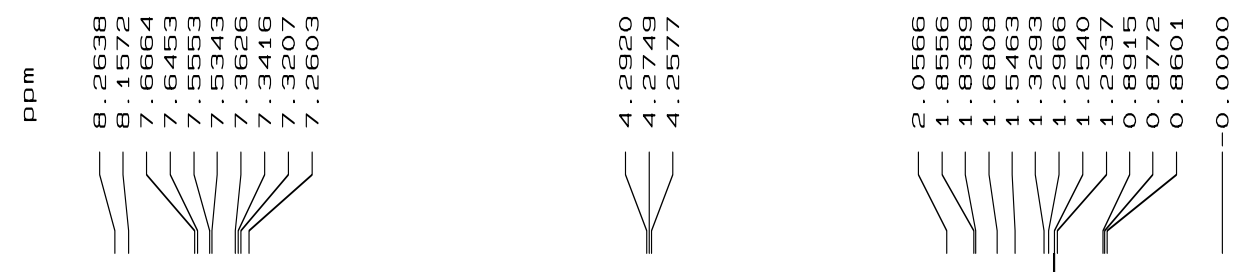

$\begin{array}{lr}\text { Current Data Parameters } \\ \text { NAME } & 1 H \\ \text { EXPNO } & 18 \\ \text { PROCNO } & 1\end{array}$

F2 - Acquisition Parameters

Date_ 20051130

Time 20.12

INSTRUM AV4OO

PAOBHD 5 m B BBO BB-1H

PULPROG 2930

TD 32768

SOLVENT COC13

NS $\quad 16$

DS 0

5WH $\quad 11185.683 \mathrm{~Hz}$

FIDRES $\quad 0.341360 \mathrm{~Hz}$

AQ $\quad 1.4647796 \mathrm{sec}$

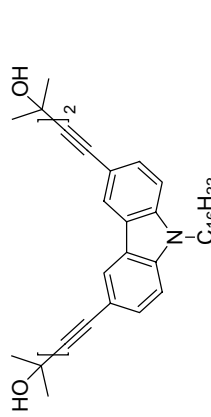

812.7

DN 44.700 usec

DE $\quad 6.00$ usec

TE $\quad 673.2 \mathrm{~K}$

$01 \quad 2.00000000 \mathrm{sec}$

MCAEST $\quad 0.00000000 \mathrm{sec}$

MCWFK $\quad 0.01500000 \mathrm{sec}$

:=:=:=:= CHANELL f1 =:=:=:=-

NOC1 $\quad$ H

P1 $\quad 10.00$ usec

PL1 $\quad-1.20 \mathrm{~dB}$

SF01 $\quad 400.1328139 \mathrm{MHz}$

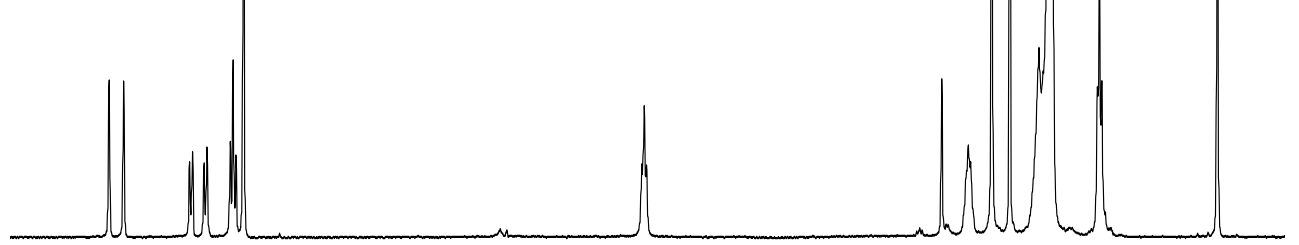

F2 - Processing parameters

SI $\quad 32768$

SF $\quad 400.1300091 \mathrm{MHz}$

WDN EM
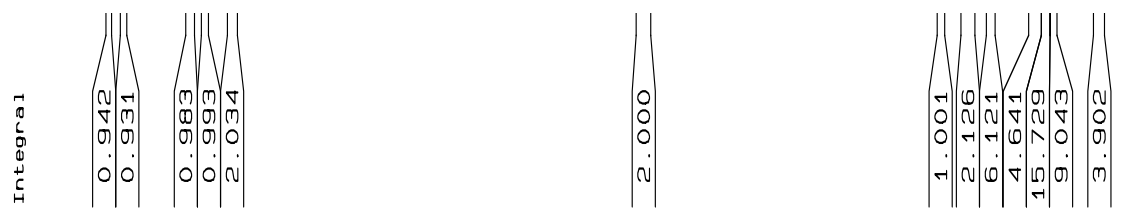

0

LB $\quad 1.00 \mathrm{~Hz}$

GB 0

PC 1.00

10 NMR plot parameters

cX $\quad 22.00 \mathrm{~cm}$

CY $\quad 12.00 \mathrm{~cm}$

Fip $\quad 9.000 \mathrm{ppm}$

F1 $\quad 3601.17 \mathrm{~Hz}$

FIP $\quad-0.500 \mathrm{ppm}$

F2 $\quad-200.07 \mathrm{~Hz}$

PPNCM $\quad 0.43182 \mathrm{ppm} / \mathrm{cm}$

HZCM $\quad 172.78342 \mathrm{~Hz} / \mathrm{cm}$

Figure S36: ${ }^{1} \mathrm{H}$ NMR spectra of $\mathbf{1 0 b}$ 

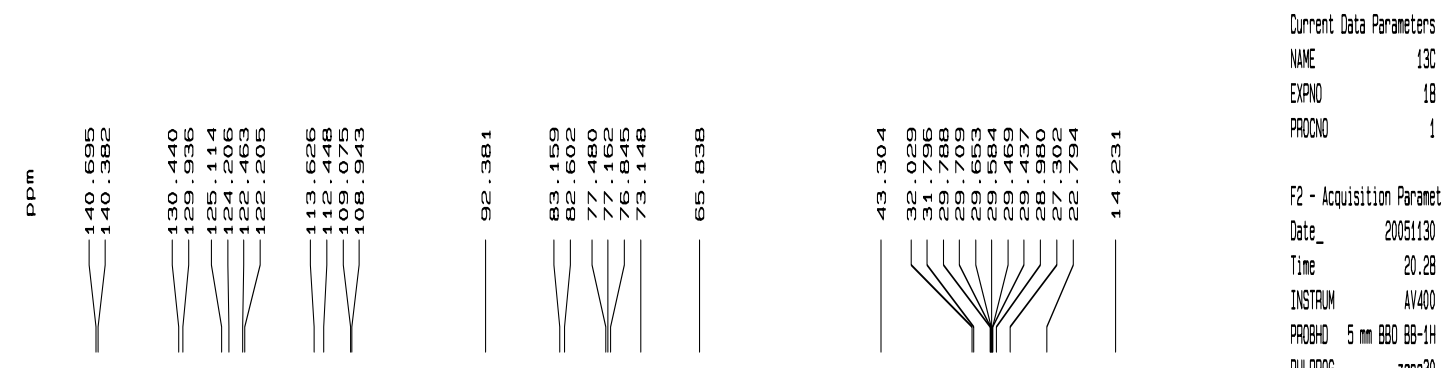

- Acoujisition Paraneters

Date_ 20051130

Tine 20.28

ISTRYY AYMOO

PPOOHO 5 ח 1 BBO B8-1H

PULPPOG zgpg30

To 65536

SOLVENT COCL13

NS 57

DS 24509.8005

FIDFES $\quad 0.373990 \mathrm{~Hz}$

Al $\quad 1.3369844 \mathrm{sec}$

Pfo $\quad 9195 . ?$

DN 20.400 useC

DE $\quad 6.00$ usec

IE $\quad 673.2 \mathrm{~K}$

012.0000000 sec

di1 $\quad 0.03000000$ sec

DELTA $1.89999998 \mathrm{SeC}$

MCREST O. OOOOOOOO SEC

MCHAK $\quad 0.01500000$ sec
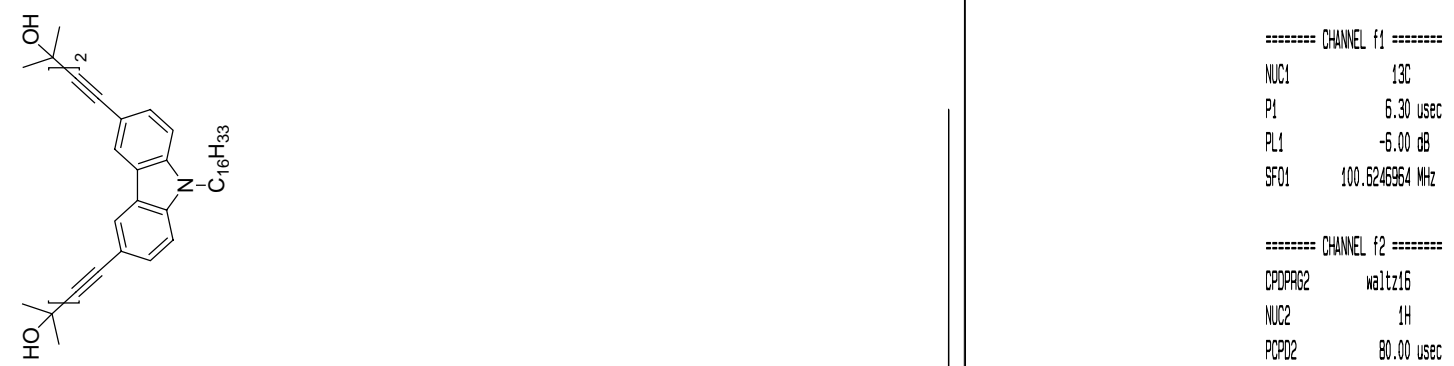

\begin{tabular}{|c|c|}
\hline 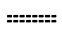 & AWELL $12==$ \\
\hline CPDPABC & waltzil6 \\
\hline NCE2 & $1 \mathrm{H}$ \\
\hline PPPD2 & 80.00 usec \\
\hline Pl2 & $-4.00 \mathrm{~dB}$ \\
\hline PL12 & $16.00 \mathrm{~dB}$ \\
\hline PL13 & $17.00 \mathrm{~dB}$ \\
\hline 9502 & $400.1336005 \mathrm{HHz}$ \\
\hline
\end{tabular}

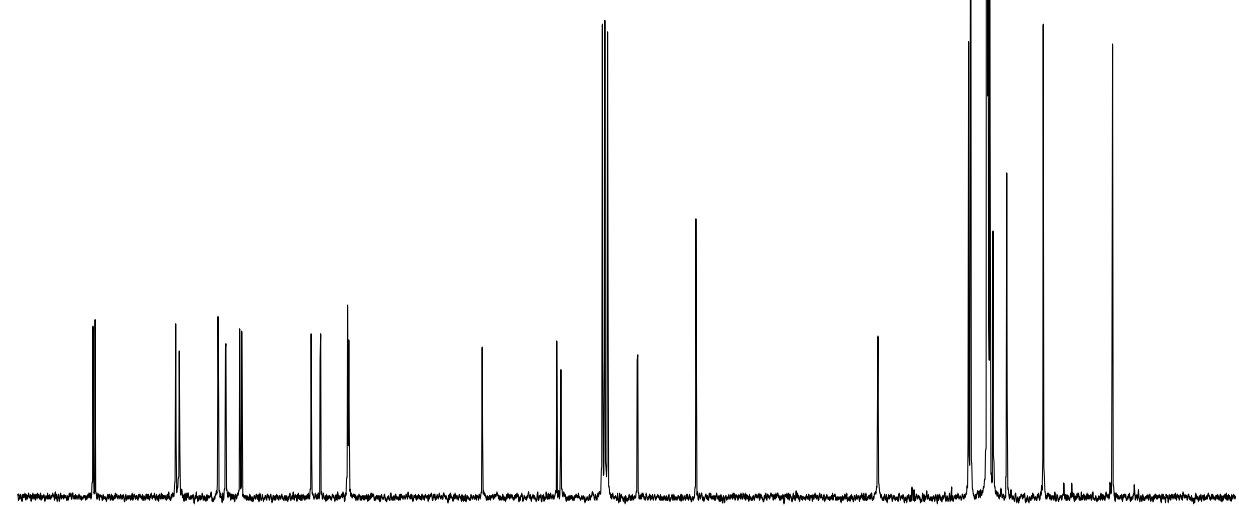

F2 - Processing paraneters

$\begin{array}{lc}\text { SI } & 32768 \\ \text { Sf } & 100.6127539 \mathrm{HZZ} \\ \text { WOH } & \text { EH } \\ \text { S98 } & 0 \\ \text { LB } & 3.00 \mathrm{~Hz} \\ \text { BB } & 0 \\ \text { PC } & 0.10\end{array}$

10 Wh plot paraneters

$\begin{array}{ll}\text { CX } & 23.00 \mathrm{~cm} \\ \text { Cy } & 10.00 \mathrm{~cm}\end{array}$

Fip $\quad 150.000$ pom

F1 $\quad 15091.91 \mathrm{~Hz}$

F.2 $\quad-1.000 \mathrm{pom}$

$\begin{array}{ll}\text { F2 } & -100.61 \mathrm{~Hz} \\ \text { PPYCH } & 6.56522 \mathrm{pon} /\end{array}$

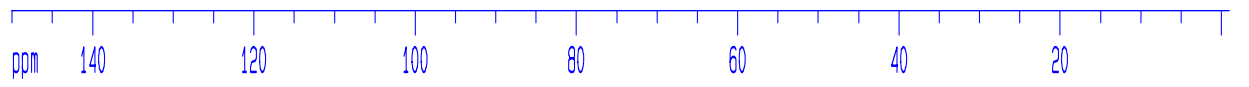

HZCH $\quad 660.54468 \mathrm{~Hz} / \mathrm{cm}$

Figure S37: ${ }^{13} \mathrm{C}$ NMR spectra of $10 b$ 

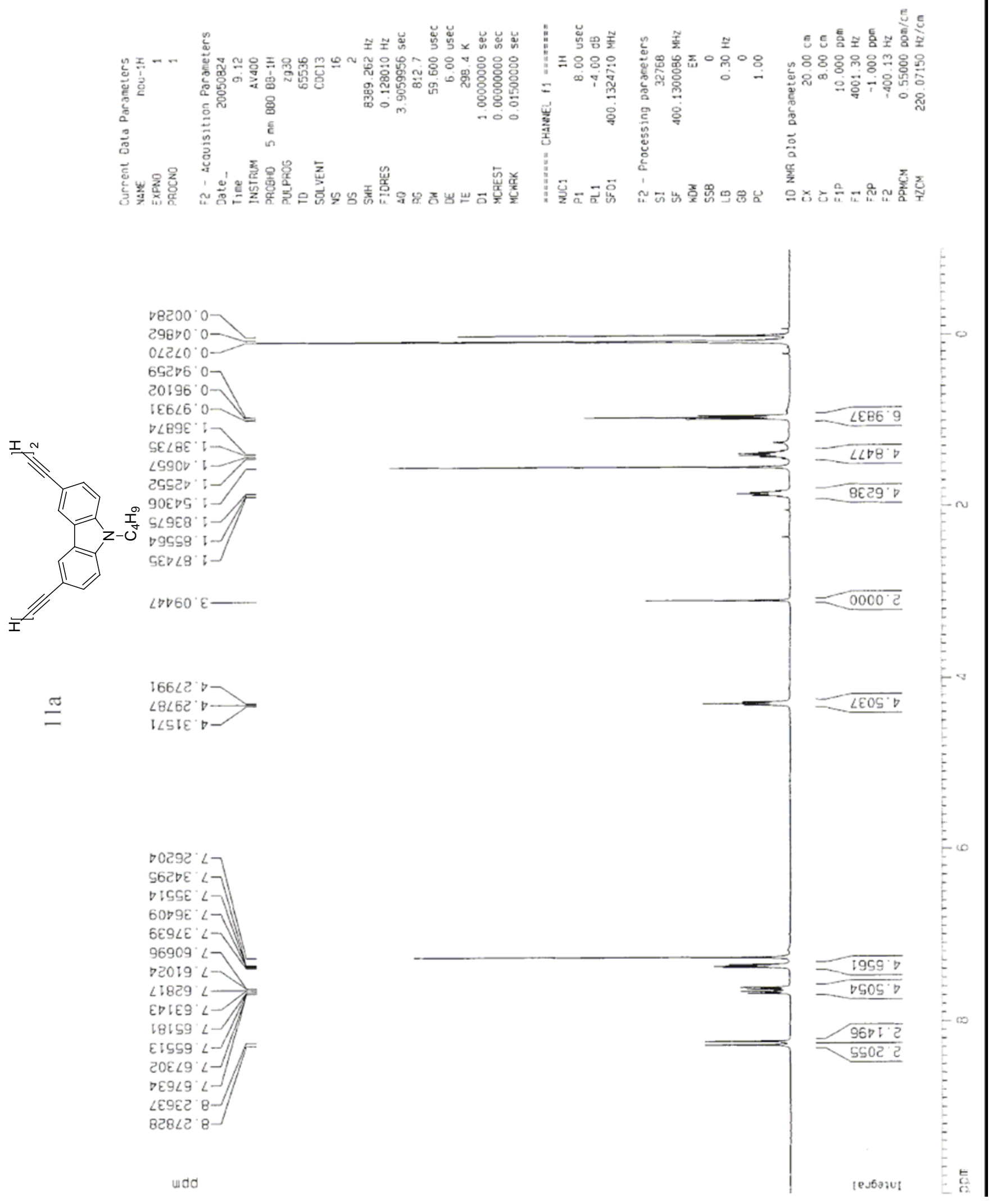

Figure S38: ${ }^{1} \mathrm{H}$ NMR spectra of 11a 

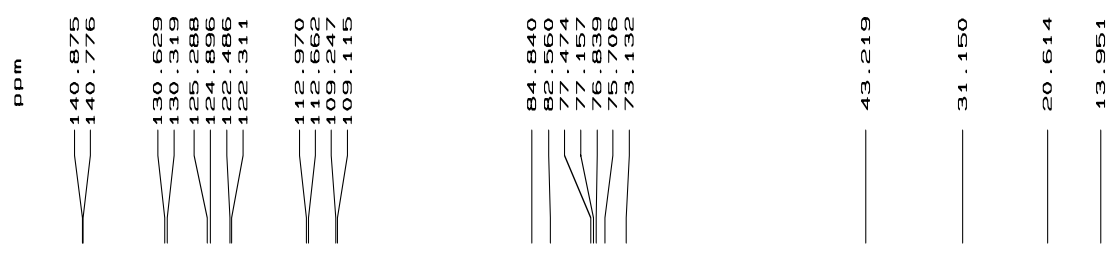

\begin{tabular}{|c|c|}
\hline \multicolumn{2}{|c|}{ Current Data Paraneters } \\
\hline VMVE & $13 C$ \\
\hline EPNO & 19 \\
\hline SPCCNO & 1 \\
\hline \multicolumn{2}{|c|}{;2 - Acouisition Paraneters } \\
\hline Jate_ & 20051214 \\
\hline Tine & 22.11 \\
\hline INSTRY & AV400 \\
\hline \multicolumn{2}{|c|}{ DPOBHO 5 m m BBO B8-1H } \\
\hline PUPPOG & zgpge30 \\
\hline To & 65536 \\
\hline SOVENT & $\mathrm{COCl}_{3}$ \\
\hline NS & 820 \\
\hline JS & 0 \\
\hline SHA & $24509.805 \mathrm{~Hz}$ \\
\hline FIDEES & $0.373990 \mathrm{~Hz}$ \\
\hline AQ & $1.3359844 \mathrm{sec}$ \\
\hline 76 & 9195.2 \\
\hline DK & 20.400 usec \\
\hline JE & 6.00 usec \\
\hline TE & $296.7 \mathrm{~K}$ \\
\hline 01 & $2.00000000 \mathrm{sec}$ \\
\hline$d 11$ & $0.03000000 \mathrm{sec}$ \\
\hline JELTA & 1.899999998 sec \\
\hline YREST & $0.00000000 \mathrm{sec}$ \\
\hline YYT & 0.0150000 \\
\hline
\end{tabular}

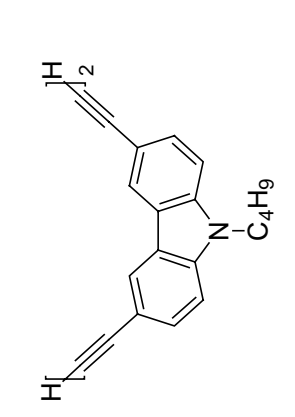

:eze:=: CHWEL f1 =:-e:=:

\begin{tabular}{|c|c|}
\hline & \\
\hline NCI & 130 \\
\hline 91 & $6.30 \mathrm{usec}$ \\
\hline$P l 1$ & $-6.00 \mathrm{~dB}$ \\
\hline $\mathrm{SFO1}$ & $100.6266964 \mathrm{MHz}$ \\
\hline :=:": & HANELL f? =:=:=:= \\
\hline CPDPAG2 & waltzil. \\
\hline NCE2 & IH \\
\hline OPPD? & 80.00 usec \\
\hline$P 2 ?$ & $-4.00 \mathrm{~dB}$ \\
\hline P.12 & $16.00 \mathrm{~dB}$ \\
\hline PL13 & $17.00 \mathrm{~dB}$ \\
\hline SFO2 & $400.1336005 \mathrm{WHz}$ \\
\hline
\end{tabular}

F2 - Processing paraneters

\begin{tabular}{|c|c|}
\hline SI & 32768 \\
\hline & $100.6127566 \mathrm{HHz}$ \\
\hline NOH & EN \\
\hline & 0 \\
\hline 8 & $3.00 \mathrm{~Hz}$ \\
\hline 60 & 0 \\
\hline C & 0.10 \\
\hline & paraneters \\
\hline r & $23.00 \mathrm{~cm}$ \\
\hline & $8.00 \mathrm{~cm}$ \\
\hline $\mathbb{1 P}$ & 150.000 קpo \\
\hline & $15091.91 \mathrm{~Hz}$ \\
\hline 20 & -1.000 pon \\
\hline & $-100.61 \mathrm{~Hz}$ \\
\hline PYC & $6.56522 \mathrm{pm} / \mathrm{cm}$ \\
\hline & $600.54462 \mathrm{~Hz} / \mathrm{cm}$ \\
\hline
\end{tabular}

Figure S39: ${ }^{13} \mathrm{C}$ NMR spectra of 11a 


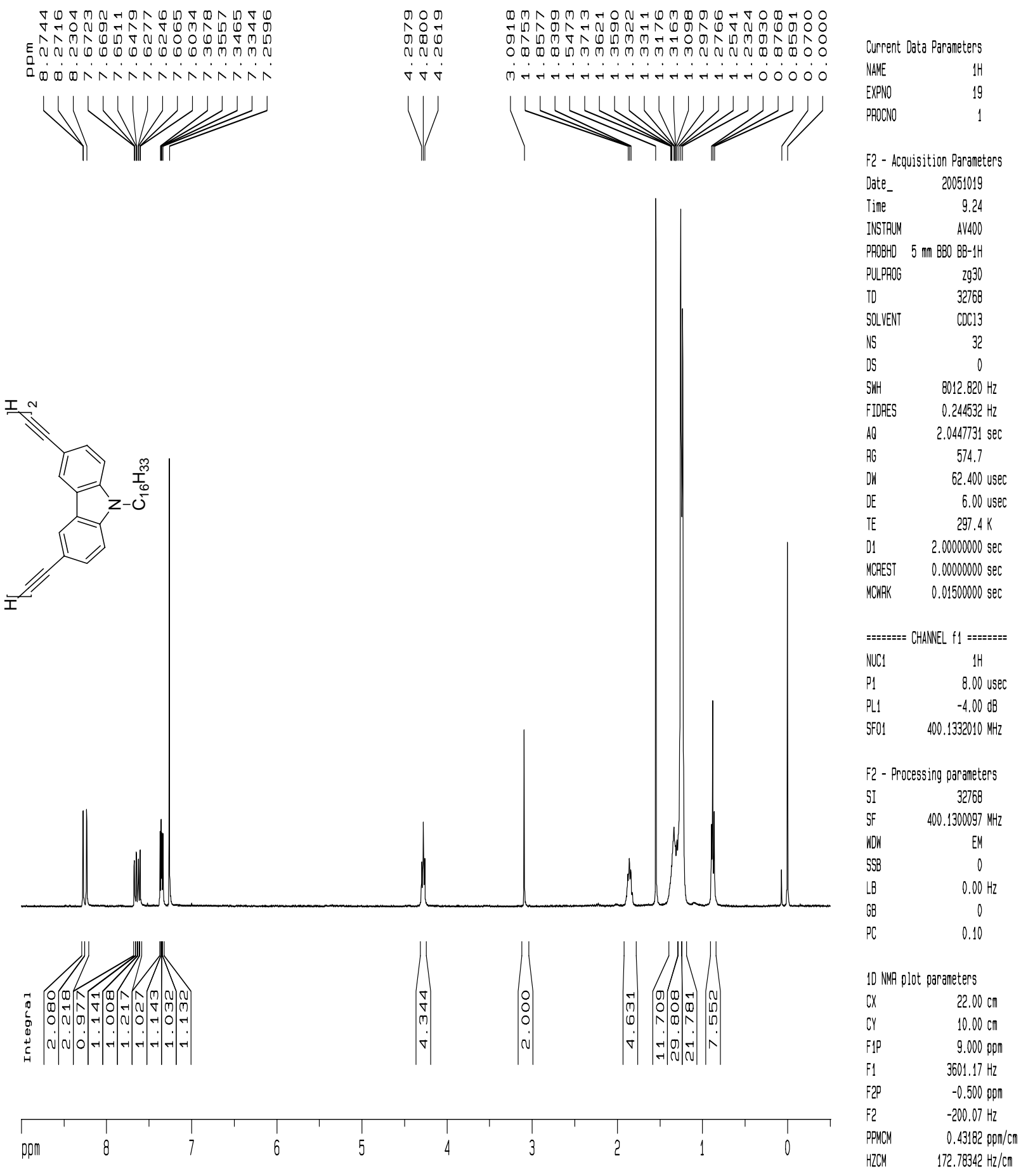

Figure S40: ${ }^{1} \mathrm{H}$ NMR spectra of $\mathbf{1 1 b}$ 

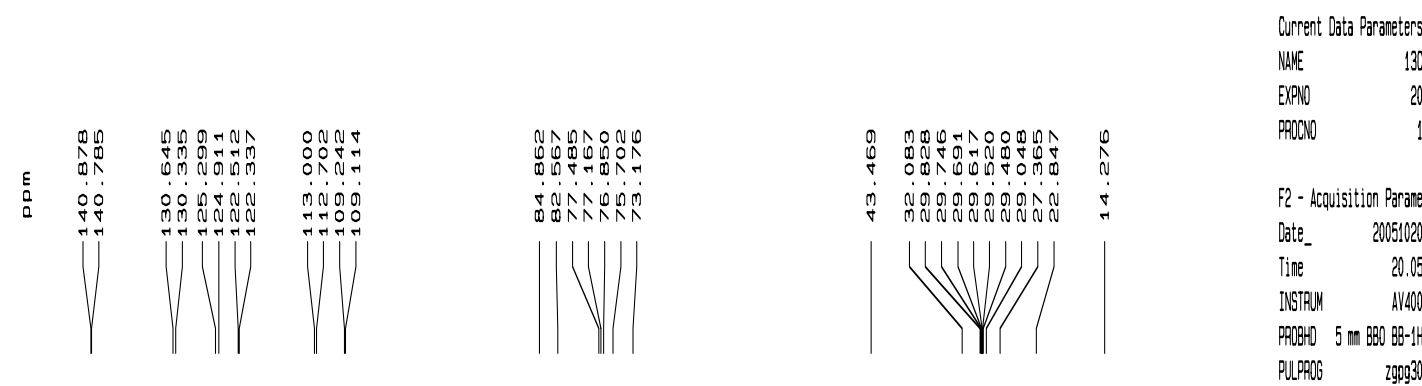

$\because 2$ - Acquisition Paraneters

Jate_ 2005102

Tine 20.05

INSTRUM AV4OO

JPOAHD $5 \mathrm{~mm}$ BBO BB-1H

गULPPOG 290930

ID 65536

3OLENT COC13

IS 2100

5以H $24509.805 \mathrm{~Hz}$

:IDPES $\quad 0.373990 \mathrm{~Hz}$

AQ $\quad 1.3359844 \mathrm{sec}$

i6 $\quad 10321.3$

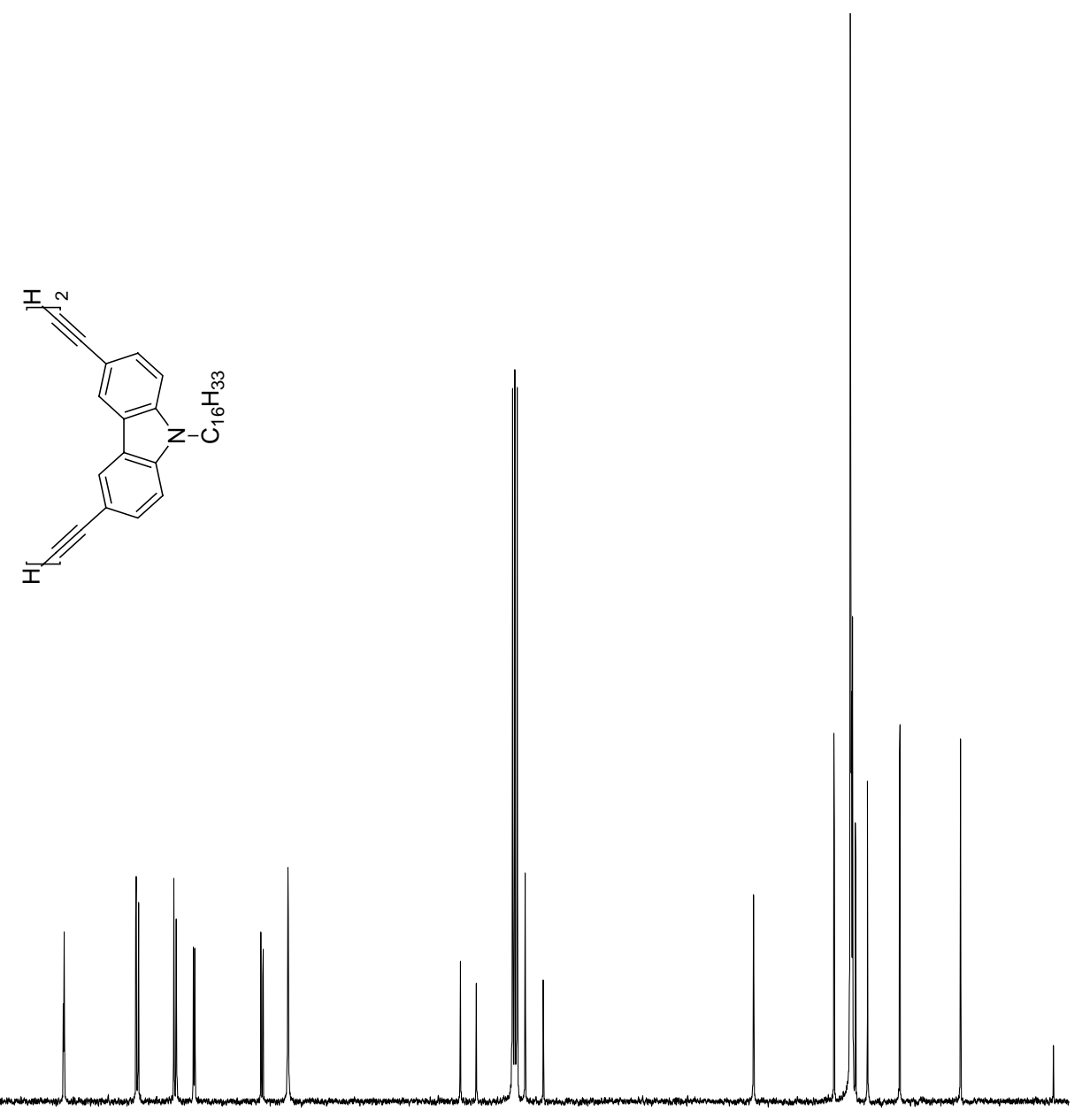

on 20.400 usec

Je $\quad 6.00$ usec

IE 298.1K

J1 $2.00000000 \mathrm{sec}$

J11 $0.03000000 \mathrm{sec}$

JELTA $1.89999998 \mathrm{sec}$

YCAEST $\quad 0.00000000 \mathrm{sec}$

YCWFK $\quad 0.01500000 \mathrm{sec}$

:=:z:=: CHANEL f1 =::=::=

$\begin{array}{lc}\text { NCI } & 13 C \\ \text { J1 } & 6.30 \text { useC } \\ \text { J.1 } & -6.00 \mathrm{~dB} \\ \text { JFO1 } & 100.62469564 \mathrm{WHZ}\end{array}$

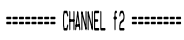

PPPPGG? Maltz16

$\begin{array}{cc}\text { NUC2 } & \text { IH } \\ \text { SCPO2 } & 80.00 \text { useC }\end{array}$

ग2? $-4,00 \mathrm{~dB}$

ग.12 $\quad 16.00 \mathrm{~dB}$

$\begin{array}{rr}2.13 & 17.00 \mathrm{~dB} \\ \mathrm{SFO2} & 400.1316005 \mathrm{NHz}\end{array}$

$: 2$ - Processing parameters

3I $\quad 32768$

35 $\quad$ 100.6127571 MHz

NOH EN

$3958 \quad 0$

.B $\quad 3.00 \mathrm{~Hz}$

$\begin{array}{lr}38 & 0 \\ x & 0.10\end{array}$

10 Nh plot paraneters

dx $\quad 23.00 \mathrm{~cm}$

$=\quad 10.00 \mathrm{~cm}$

$15091.91 \mathrm{~Hz}$

$=2 p \quad-1.000$ ppon

$\therefore \quad-100.61 \mathrm{~Hz}$

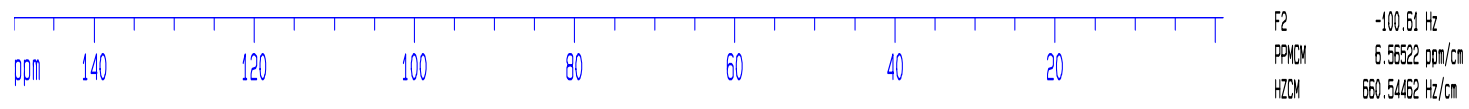

Figure S41: ${ }^{13} \mathrm{C}$ NMR spectra of $\mathbf{1 1 b}$ 


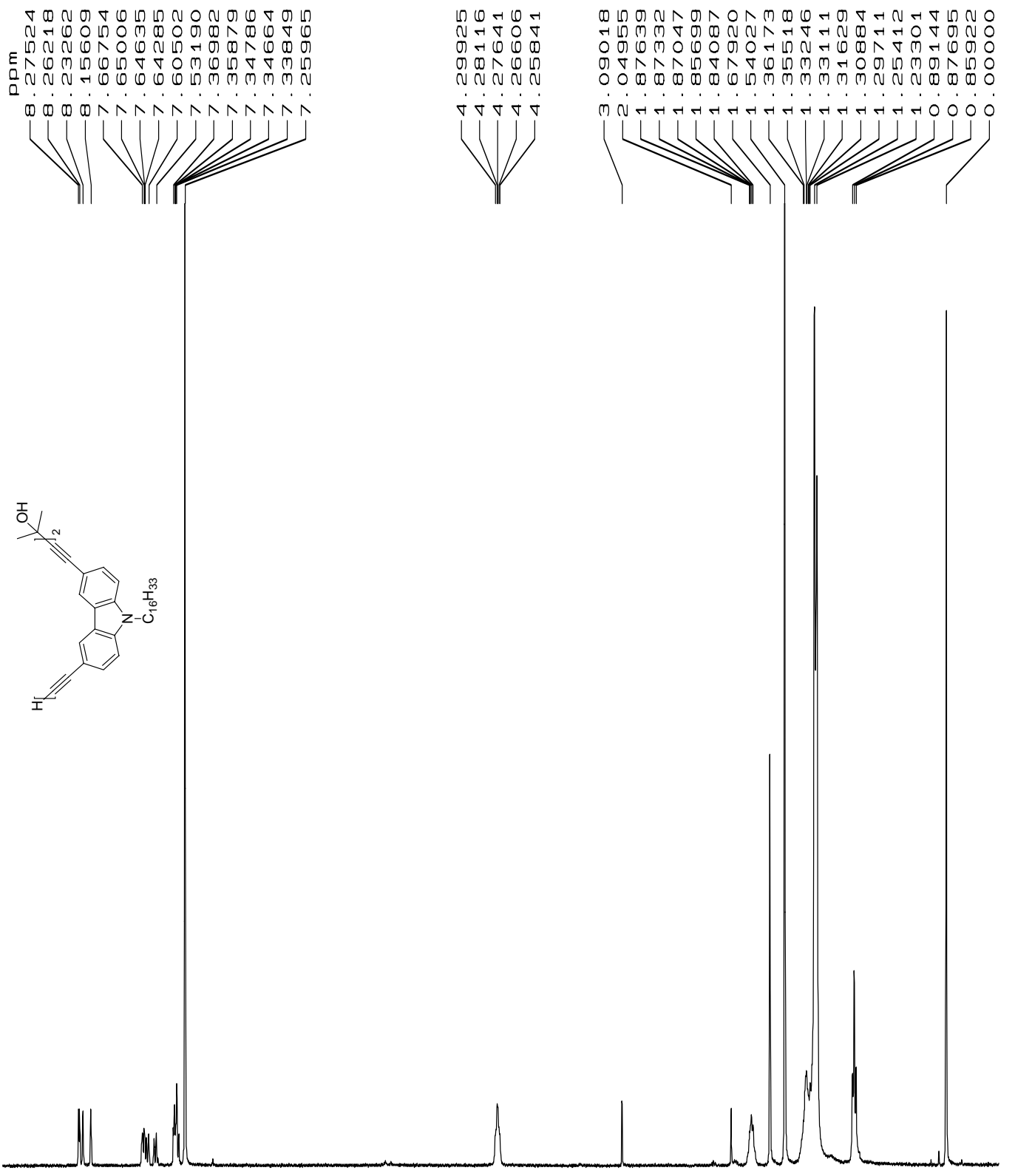

Current Data Parameters

NAME $\quad 1 H$

EXPNO 12

DROCNO 1

F2 - Acquisition Paraneters

Jate_ $\quad 20060520$

Time $\quad 12.33$

INSTRUM AV4OO

PROBHO $5 \mathrm{~mm}$ BBO BB-1H

PULPROG $\quad 2930$

TO 32768

SOLVENT COCI3

NS 64

JS 0

5WH $\quad 11990.407 \mathrm{~Hz}$

FIDRES $\quad 0.365918 \mathrm{~Hz}$

AQ $\quad 1.3664756 \mathrm{sec}$

76 $\quad 1149.4$

JW $\quad 41.700$ usec

JE $\quad 6.00$ usec

TE $298.8 \mathrm{~K}$

J1 $2.00000000 \mathrm{sec}$

MCEEST $\quad 0.00000000 \mathrm{sec}$

YCWRK $\quad 0.01500000 \mathrm{sec}$

=:z=:=:= CHAWEL $f 1$ =:=z:=:=

NUC1 $\quad$ H

P1 12.10 usec

PL1 $\quad 0.00 \mathrm{~dB}$

5F01 400.1322455 $\mathrm{NHz}$

¡2 - Processing parameters

SI $\quad 32768$

SF $\quad 400.1300097 \mathrm{MHz}$

NON EM

SSB $\quad 0$

LB $\quad 0.00 \mathrm{~Hz}$

$B B \quad O$

PC $\quad 0.10$

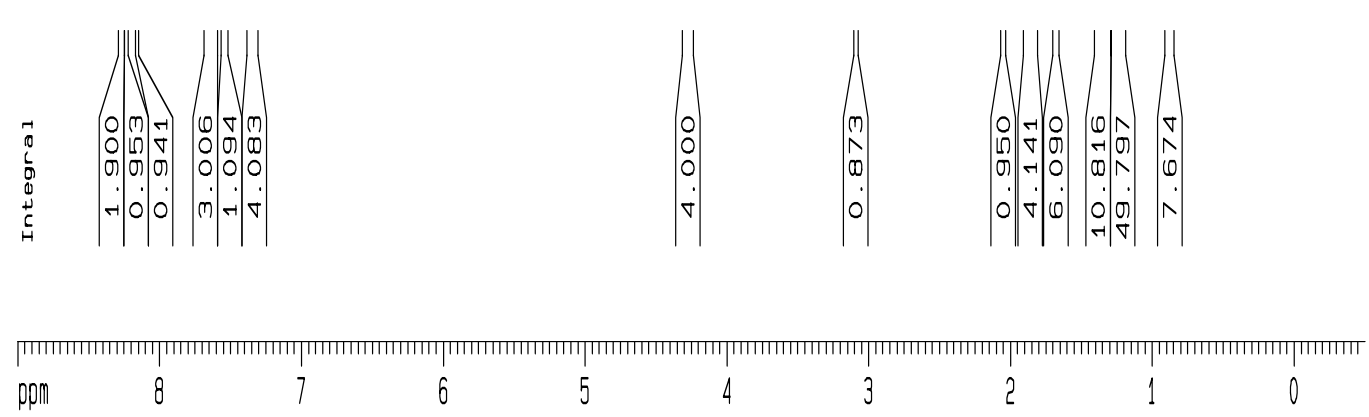

10 NMR plot parameters

CX $\quad 22.00 \mathrm{~cm}$

CY $\quad 15.00 \mathrm{~cm}$

$=1 \mathrm{p} \quad 9.000 \mathrm{ppm}$

F1 $\quad 3601.17 \mathrm{~Hz}$

$=200$

$=2 \quad-200.07 \mathrm{~Hz}$

JPMCM $\quad 0.43182 \mathrm{pDm} / \mathrm{cm}$

HZCM $\quad 172.78342 \mathrm{~Hz} / \mathrm{cm}$

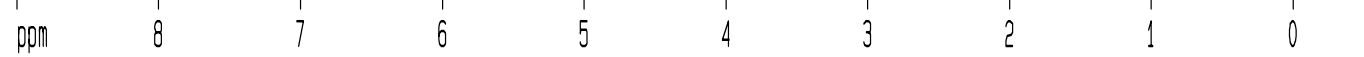

Figure S42: ${ }^{1} \mathrm{H}$ NMR spectra of $\mathbf{1 2}$ 

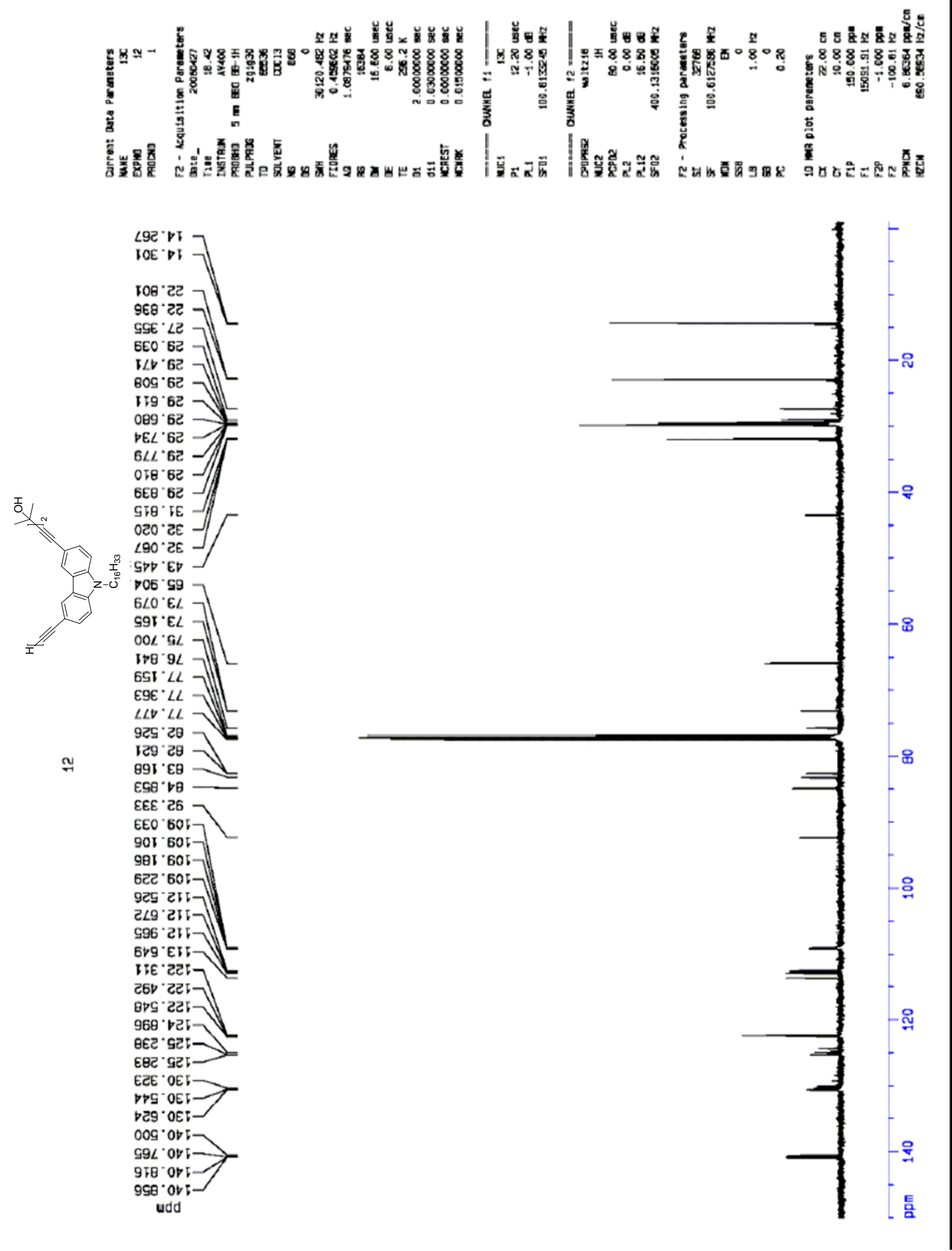

Figure S43: ${ }^{13} \mathrm{C}$ NMR spectra of $\mathbf{1 2}$ 


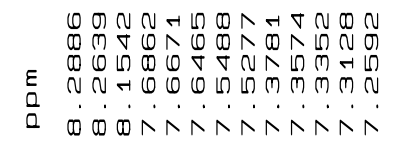

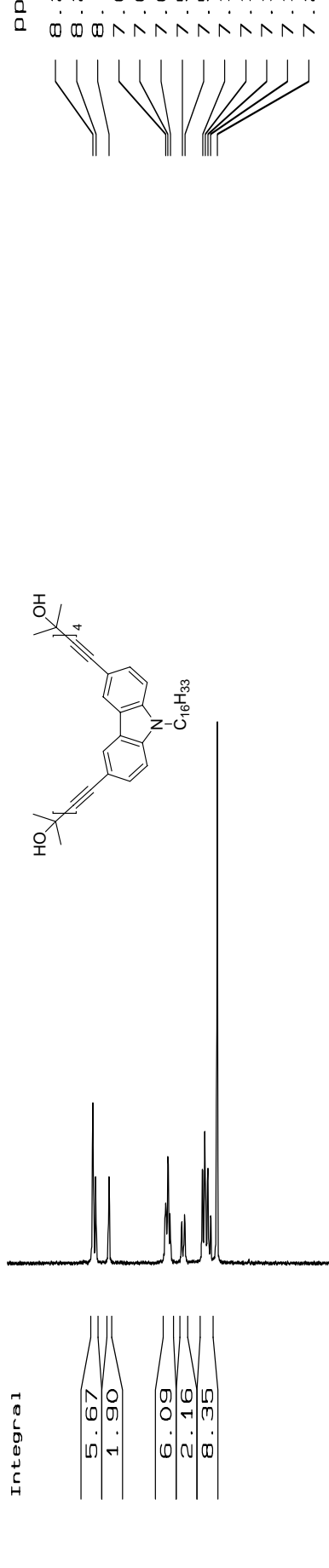

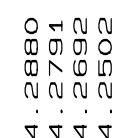

$\nabla \nabla \nabla \nabla$
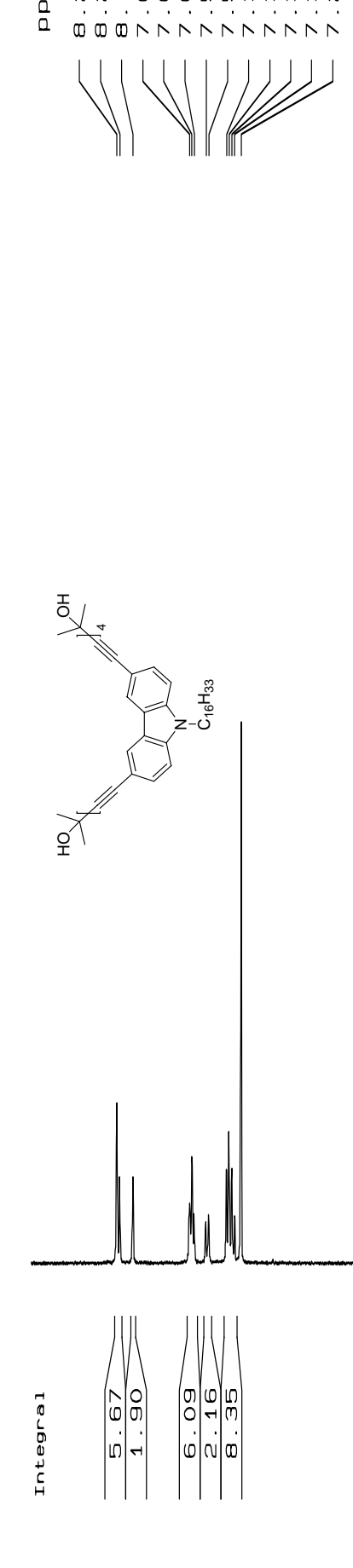

$\lambda$

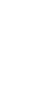

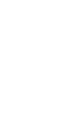

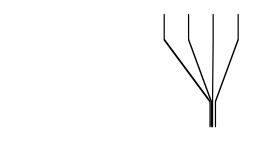

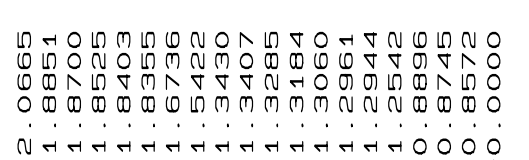

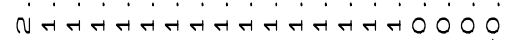

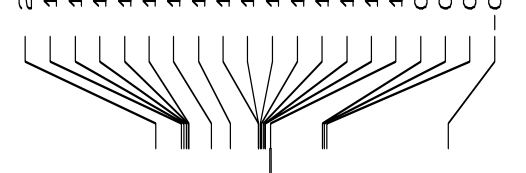

F2 - Acquisition Paraneters

Date_ $\quad 20060520$

Tine $\quad 12.44$

INSTHUM AVMOO

PROBHO 5 ח ח BBO BB-1H

PULPPOG 2030

TO 32768

SOLENT COCL13

NS $\quad 16$

DS 0

5HW $\quad 11990.407 \mathrm{~Hz}$

FIDRES $\quad 0.365918 \mathrm{~Hz}$

AQ $\quad 1.3664756 \mathrm{sec}$

HG $\quad 645.1$

DN 41.700 usec

DE $\quad 6.00$ usec

TE $298.8 \mathrm{~K}$

$01 \quad 2.00000000 \mathrm{sec}$

MCEEST $\quad 0.00000000$ SeC

MCHRK $0.01500000 \mathrm{sec}$

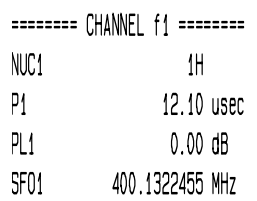

F2 - Processing paraneters

SI 32768

SF $\quad 400.1300097$ MHz

NOH EM

$598 \quad 0$

LB $\quad 0.00 \mathrm{~Hz}$

$G B \quad O$

$P C \quad 0.10$

10 NMR plot paraneters

cX $\quad 22.00 \mathrm{~cm}$

CY $\quad 12.00 \mathrm{~cm}$

Fip $\quad 9.000$ pon

F1 $\quad 3601.17 \mathrm{~Hz}$

F2P $\quad-0.500$ pp

F2 $\quad-200.07 \mathrm{~Hz}$

PPYCY $\quad 0.43182 \mathrm{pDn} / \mathrm{Cm}$

HZCM $\quad 172.78342 \mathrm{~Hz} / \mathrm{cm}$

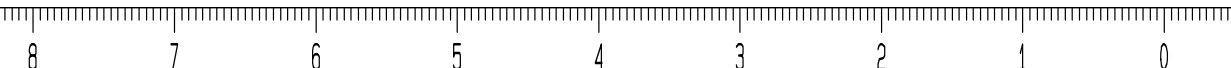

Figure S44: ${ }^{1} \mathrm{H}$ NMR spectra of 13 

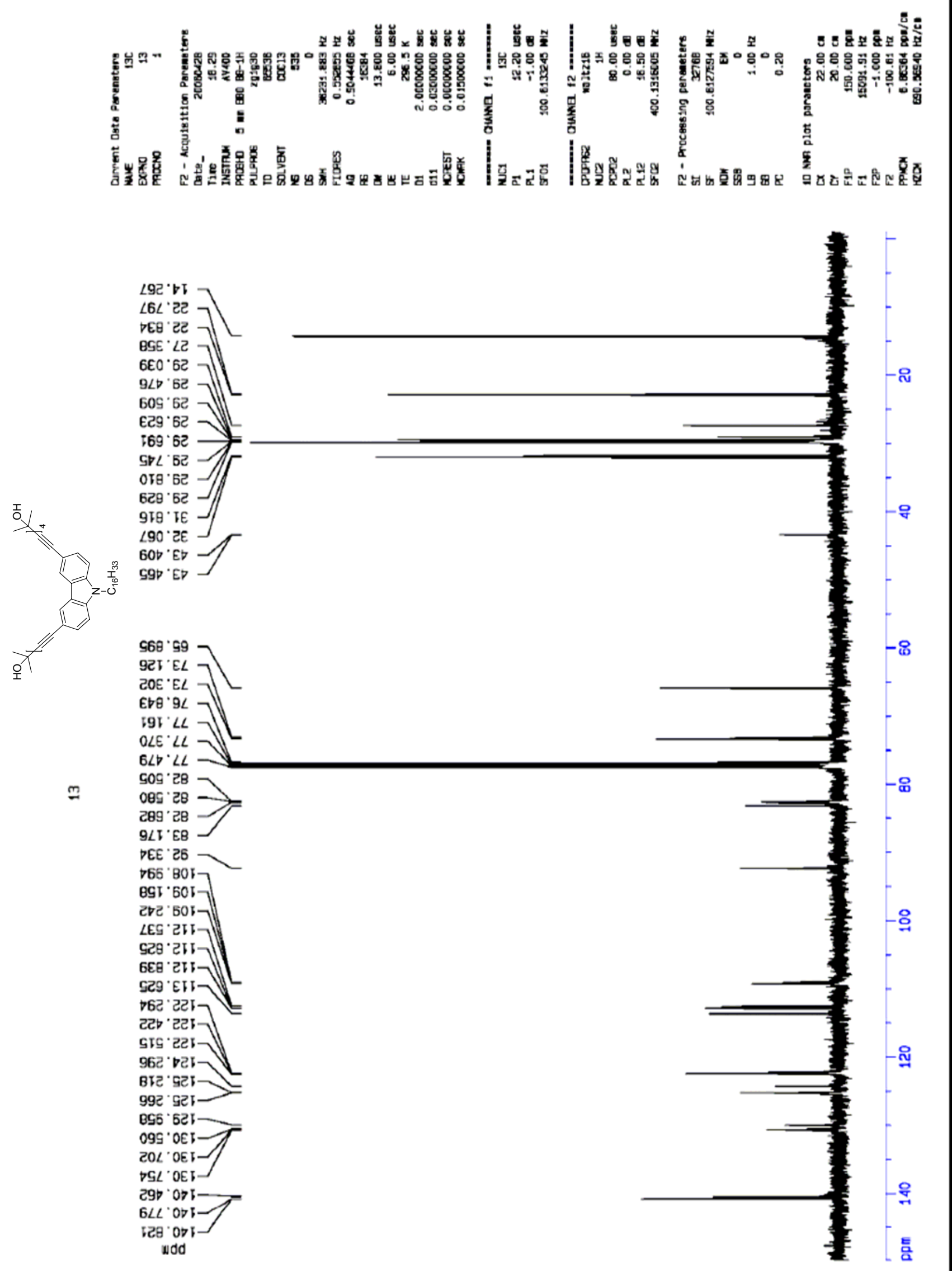

Figure S45: ${ }^{13} \mathrm{C}$ NMR spectra of $\mathbf{1 3}$ 


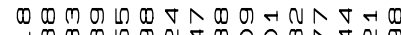

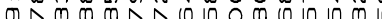
E N $\sim 0006000 \mathrm{mmmm}$

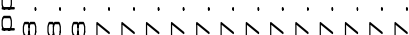

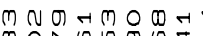

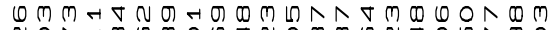

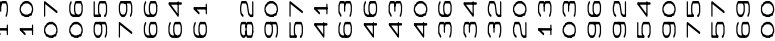

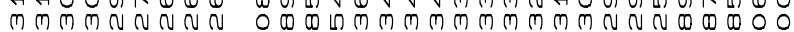

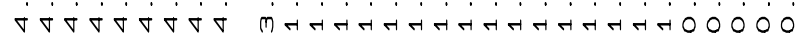

Current Data Paraneters

EXPNO

PROCNO

¡2 - Acquisition Parameters

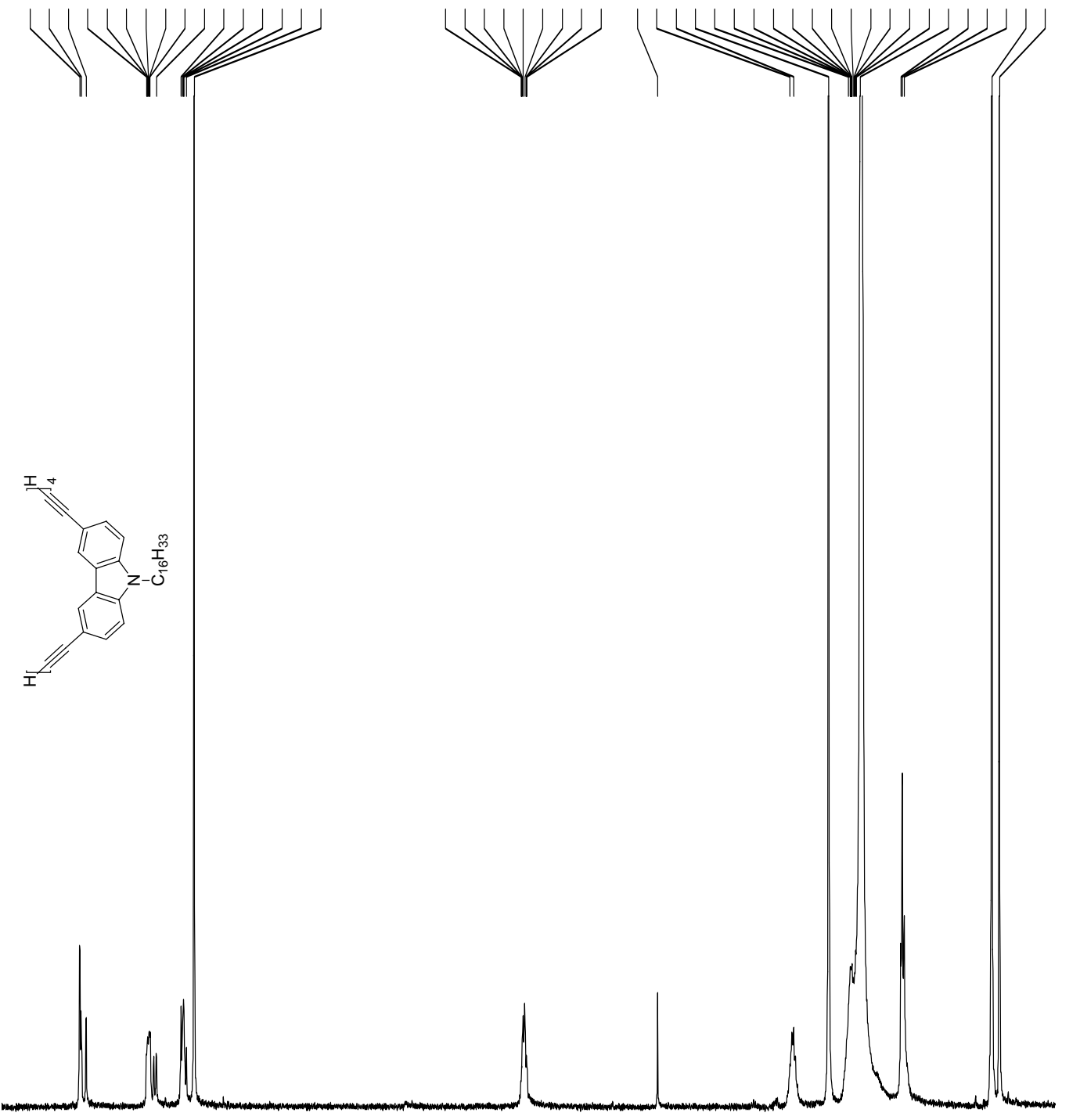

Date 20060520

Time $\quad 12.52$

INSTRUM AVAOO

PROBHO 5 ח ח BBO BB-1H

PULPROG 2930

TO 32768

SOLENT COC13

NS 16

J5 0

SWH $\quad 11990.407 \mathrm{~Hz}$

FIDRES $\quad 0.365918 \mathrm{~Hz}$

AQ $\quad 1.3664756 \mathrm{sec}$

76 $\quad 574.7$

DN $\quad 41.700$ usec

JE $\quad 6.00$ usec

TE $\quad 298.7 \mathrm{~K}$

$01 \quad 2.00000000 \mathrm{sec}$

YCREST $\quad 0.00000000 \mathrm{sec}$

VCWFK $\quad 0.01500000 \mathrm{sec}$

=:=z:=:= CHAWEL $f 1$ =:=:=:=s

$\begin{array}{lr}\text { HUC1 } & \text { IH } \\ \text { D1 } & 12.10 \text { usec } \\ \text { PL1 } & 0.00 \mathrm{~dB} \\ \text { SFO1 } & 400.1322455 \mathrm{NHz}\end{array}$

:2 - Processing parameters

SI 32768

SF $400.1300094 \mathrm{NHz}$

NON EM

SSB $\quad 0$

LB $\quad 0.00 \mathrm{~Hz}$

$G B \quad 0$

ग $\quad 0.10$

$\begin{array}{lc}10 \text { NMR plot parameters } \\ C X & 22.00 \mathrm{~cm} \\ C Y & 15.00 \mathrm{~cm} \\ -1 \mathrm{P} & 9.000 \mathrm{ppm} \\ -1 & 3601.17 \mathrm{~Hz} \\ =2 \mathrm{P} & -0.500 \mathrm{ppm} \\ -2 & -200.07 \mathrm{~Hz} \\ \text { JPNCM } & 0.43182 \mathrm{ppm} / \mathrm{cm} \\ \text { HZCM } & 172.78342 \mathrm{~Hz} / \mathrm{cm}\end{array}$

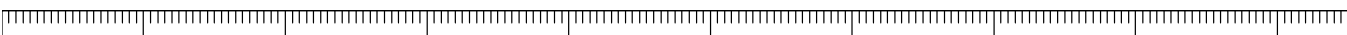

$\begin{array}{lllllllll}\text { ppm } & 8 & 7 & 6 & 5 & 4 & 3 & 2 & 1\end{array}$

Figure S46: ${ }^{1} \mathrm{H}$ NMR spectra of 14 

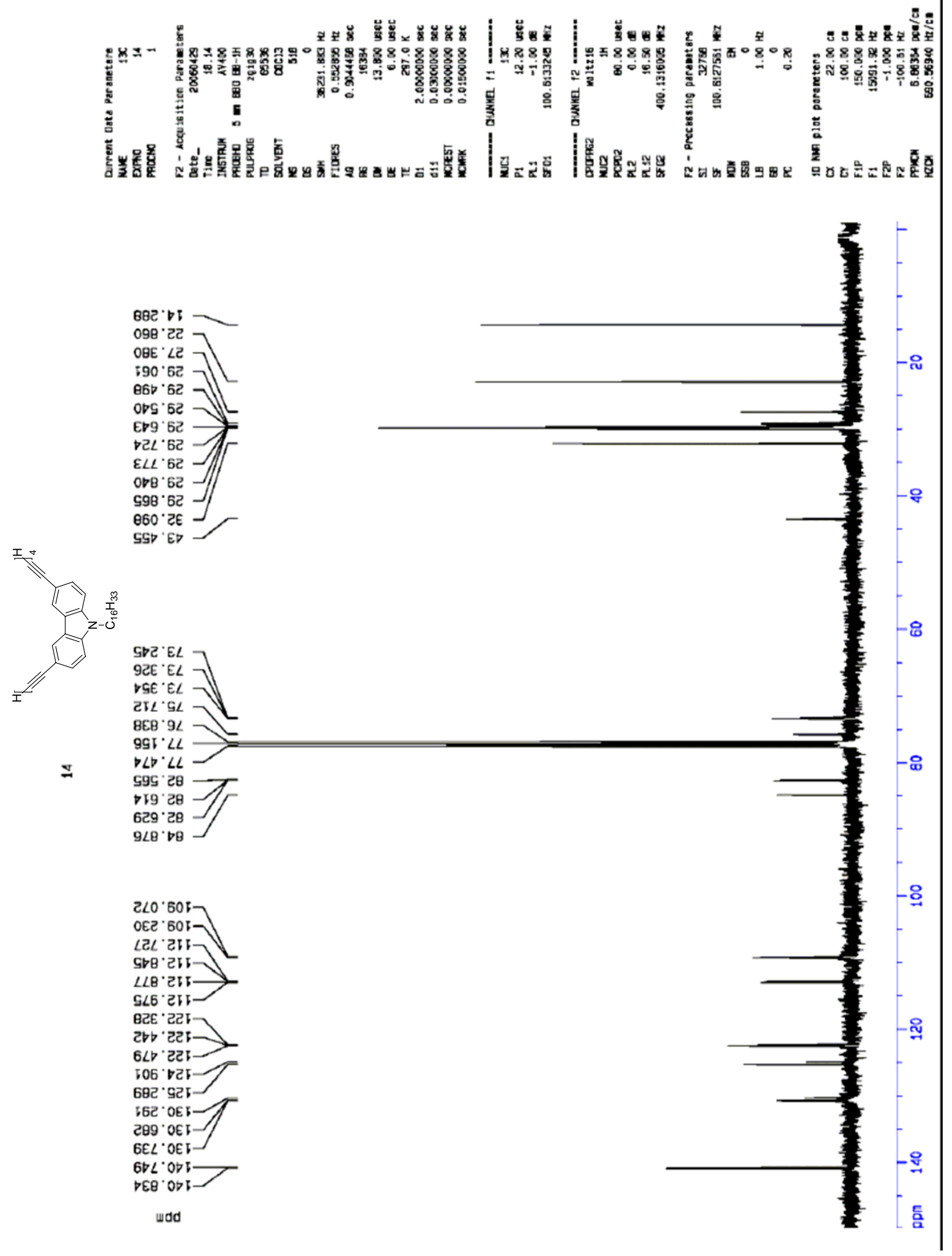

Figure S47: ${ }^{13} \mathrm{C}$ NMR spectra of $\mathbf{1 4}$ 


\section{Cyclic voltammetry curves.}

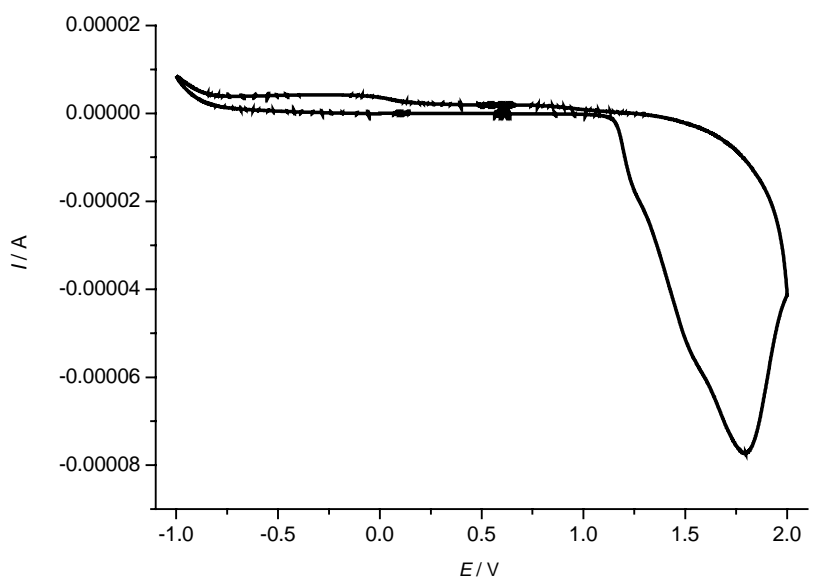

Figure S48: Cyclic voltammetry curves of 14

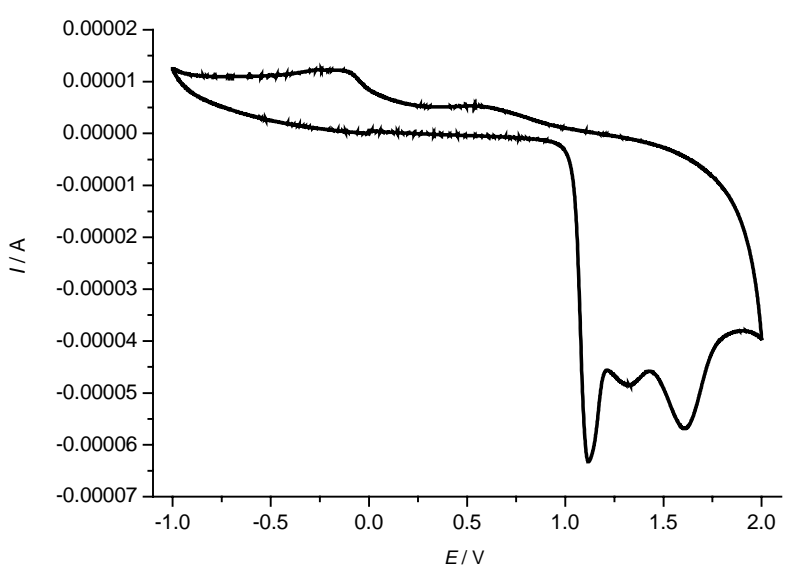

Figure S49: Cyclic voltammetry curves of $\mathbf{1 b}$

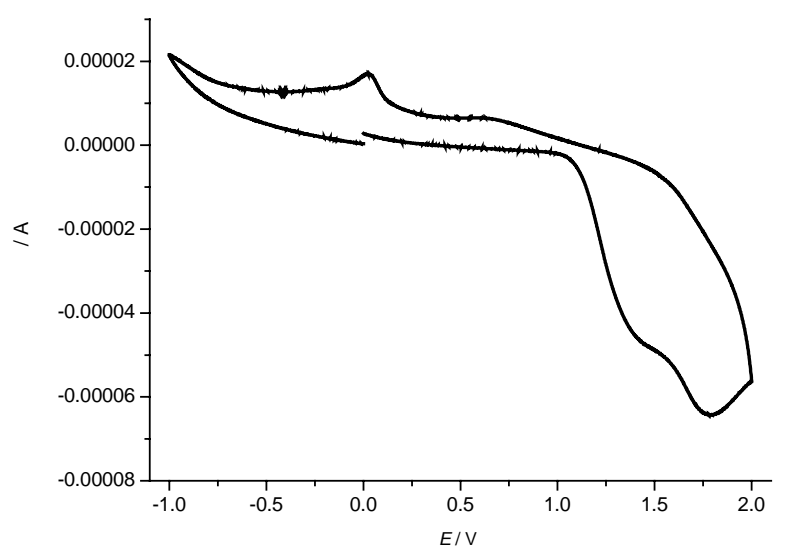

Figure S50: Cyclic voltammetry curves of $\mathbf{2 b}$

\section{HRMS.}




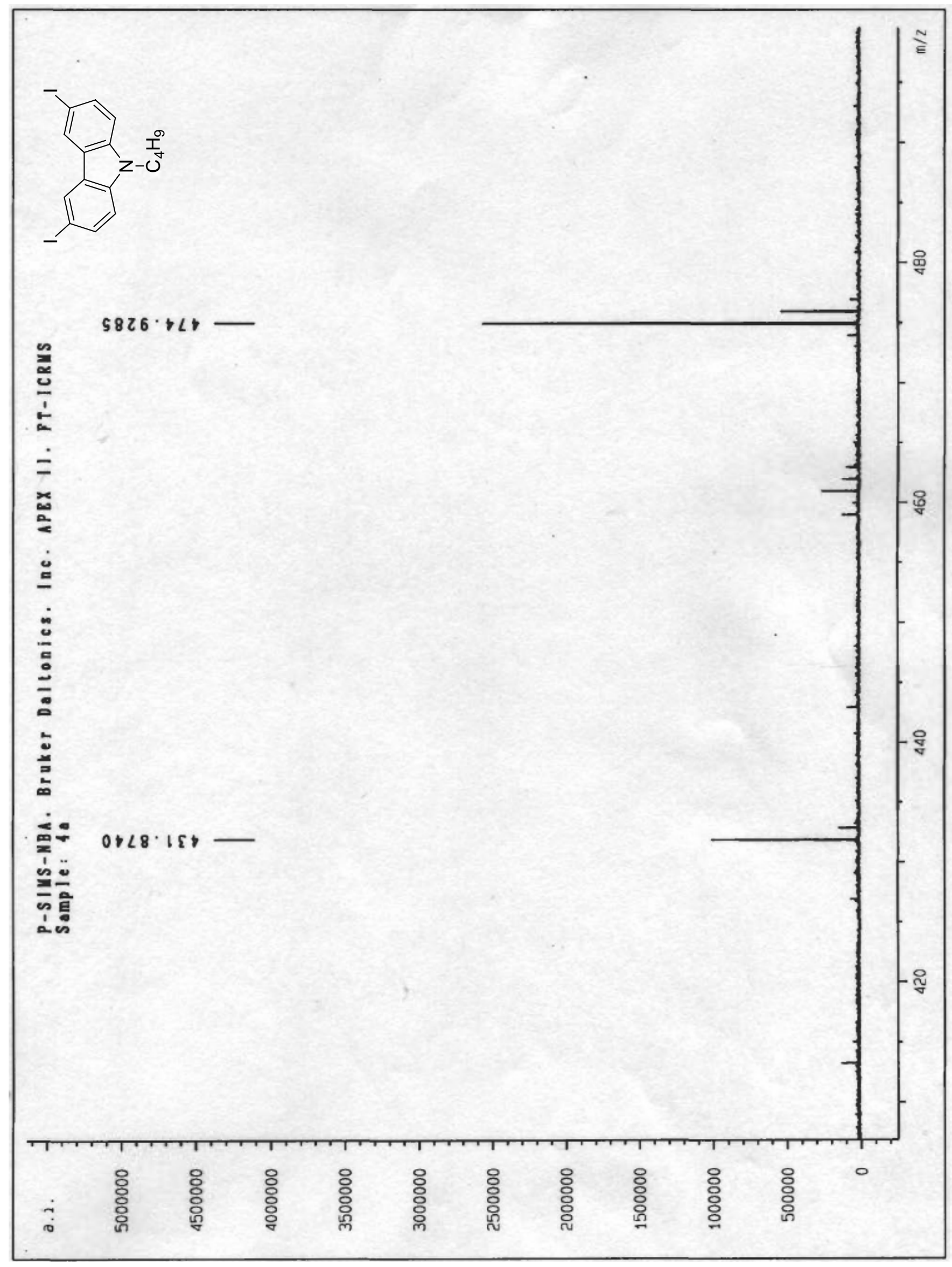

Figure S51: HRMS of 4a 


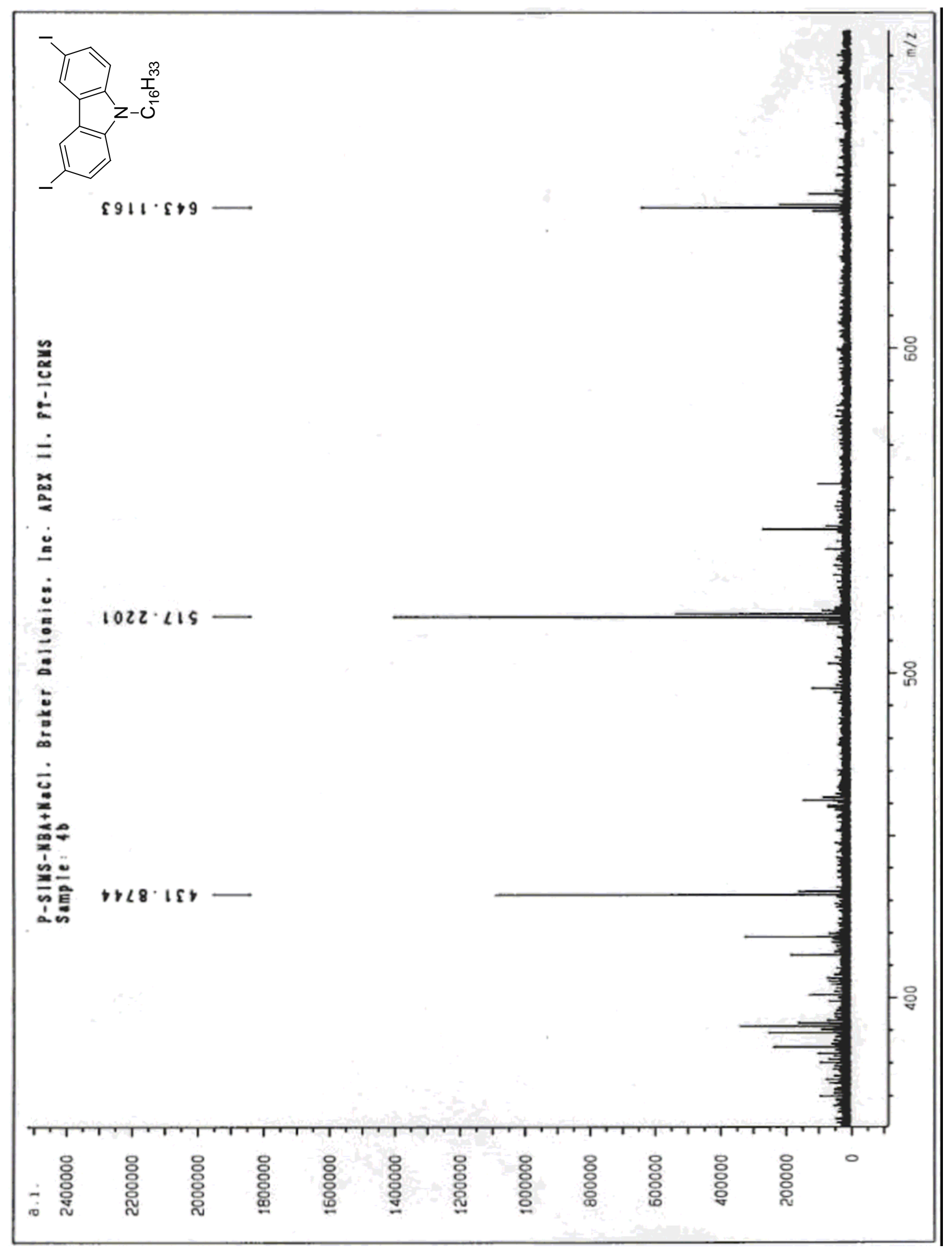

Figure S52: HRMS of $\mathbf{4 b}$ 


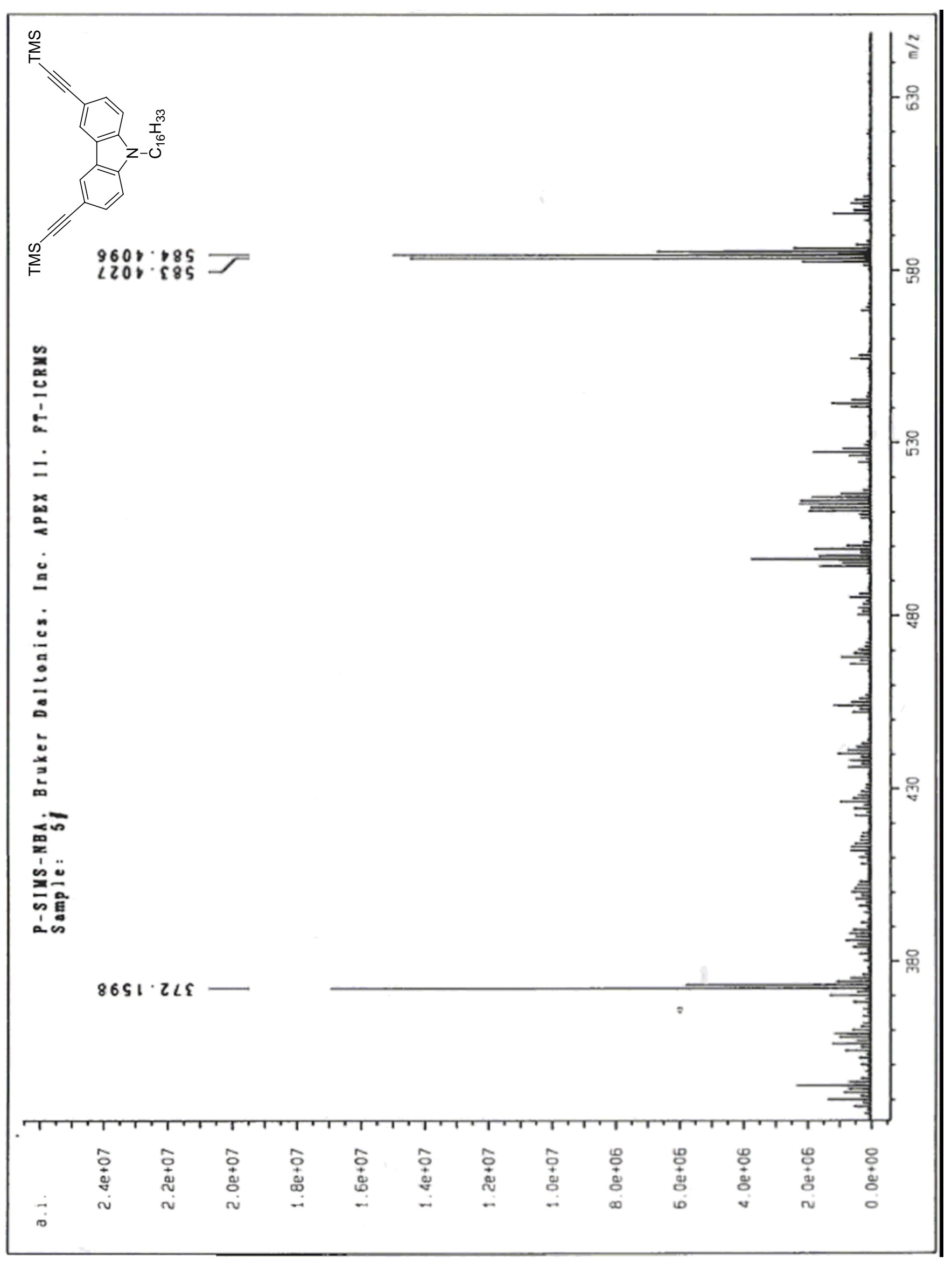

Figure S53: HRMS of $\mathbf{5 b}$ 


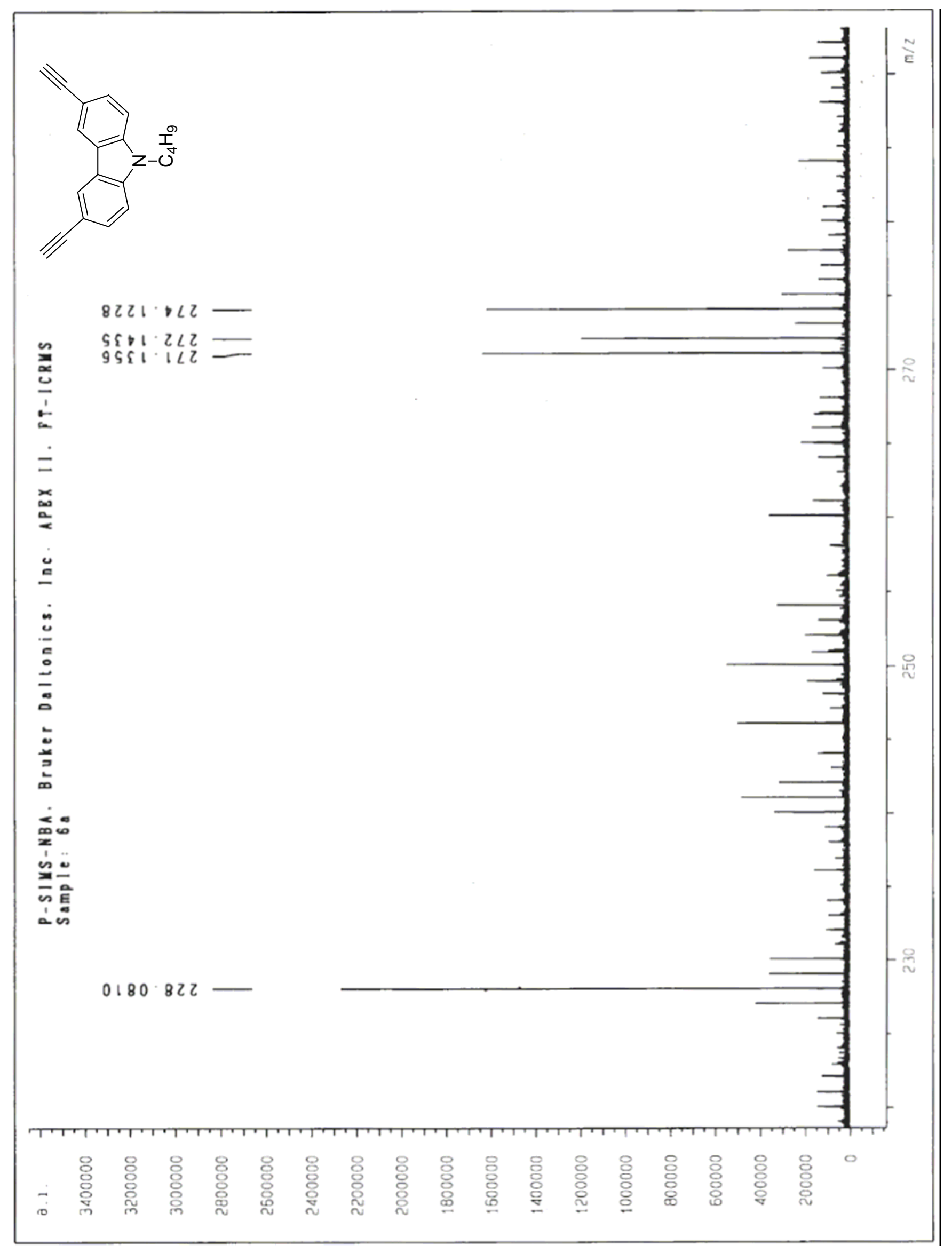

Figure S54: HRMS of $\mathbf{6 a}$ 


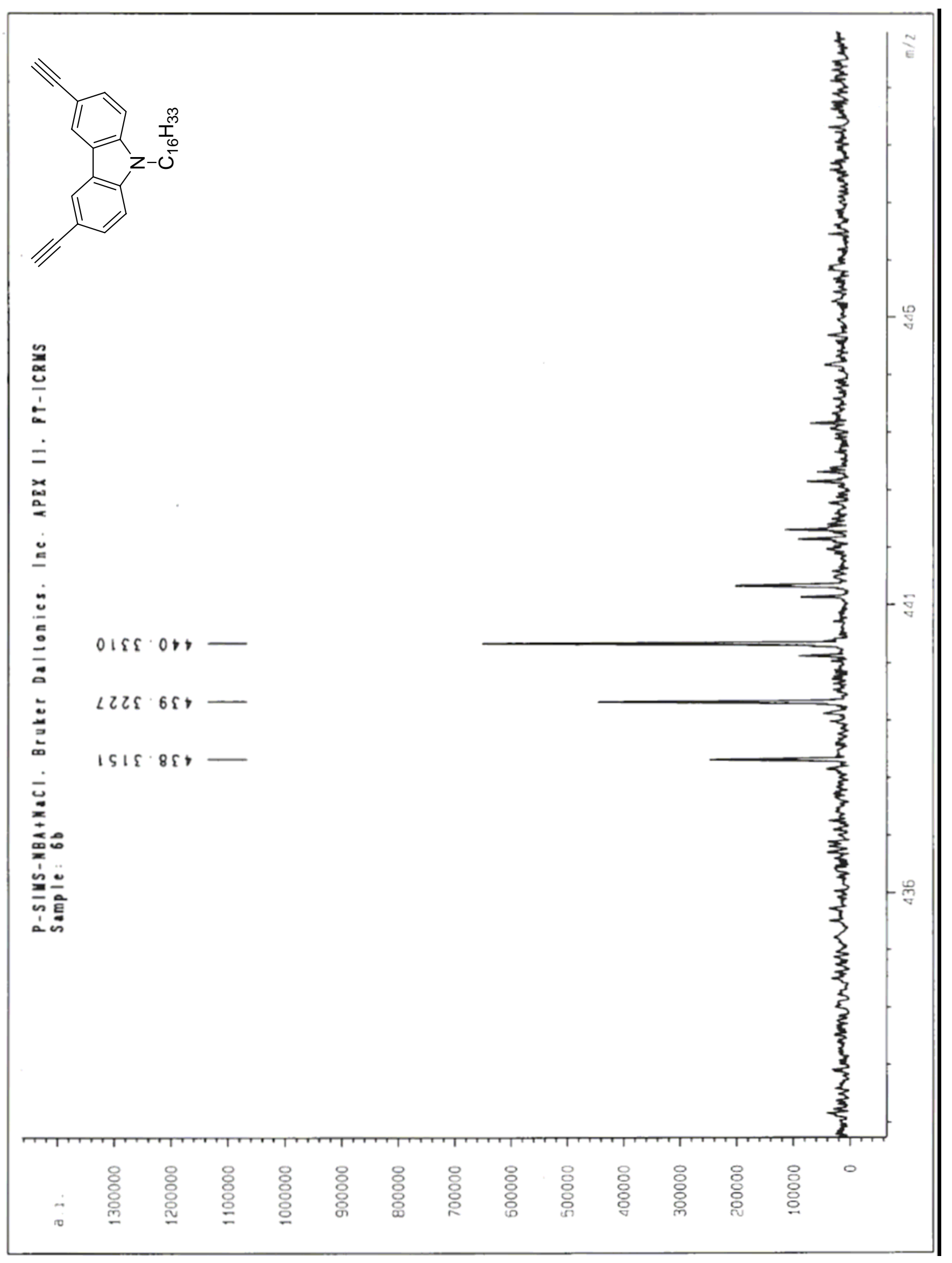

Figure S55: HRMS of $\mathbf{6 b}$ 


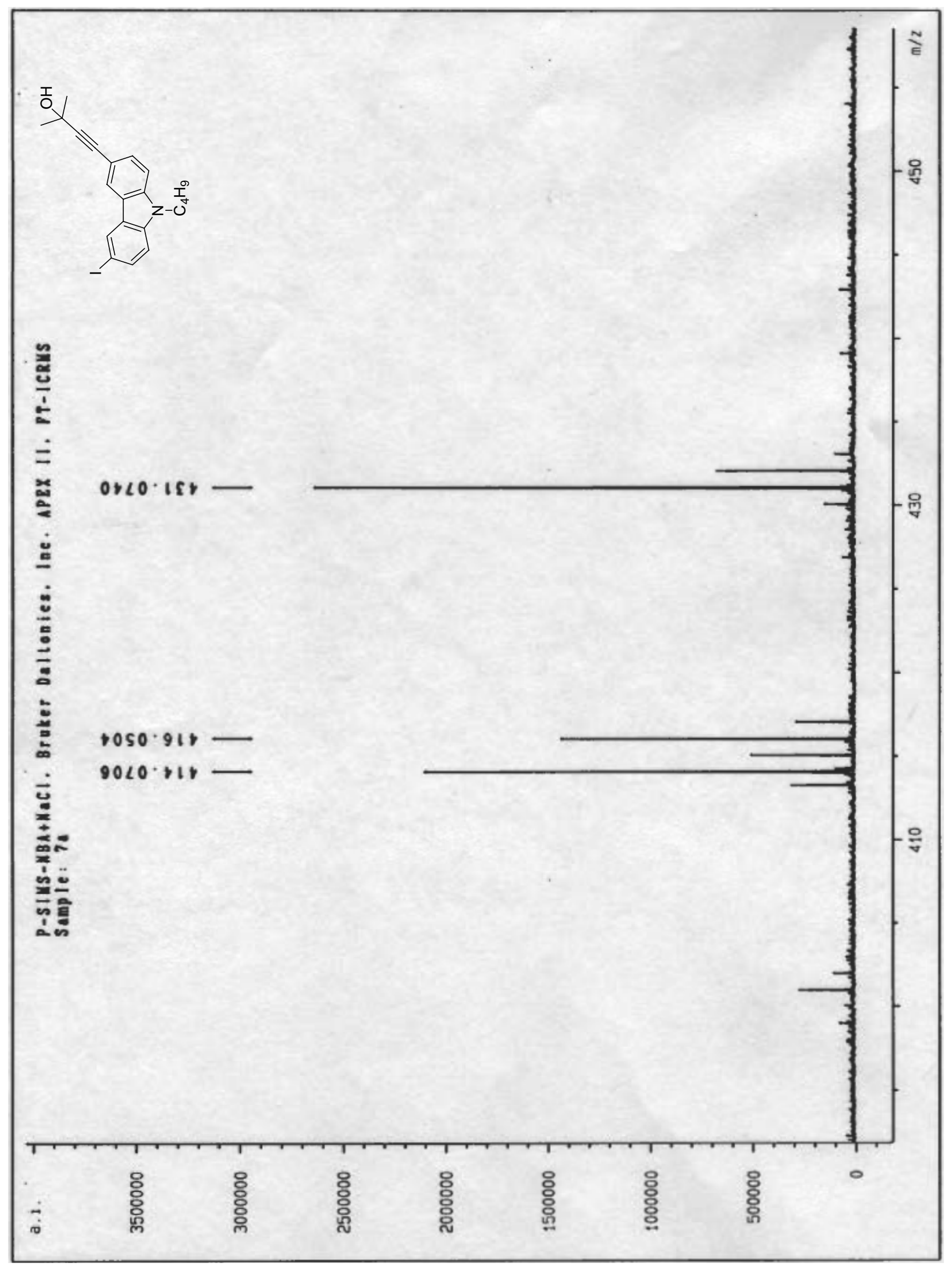

Figure S56: HRMS of 7a 


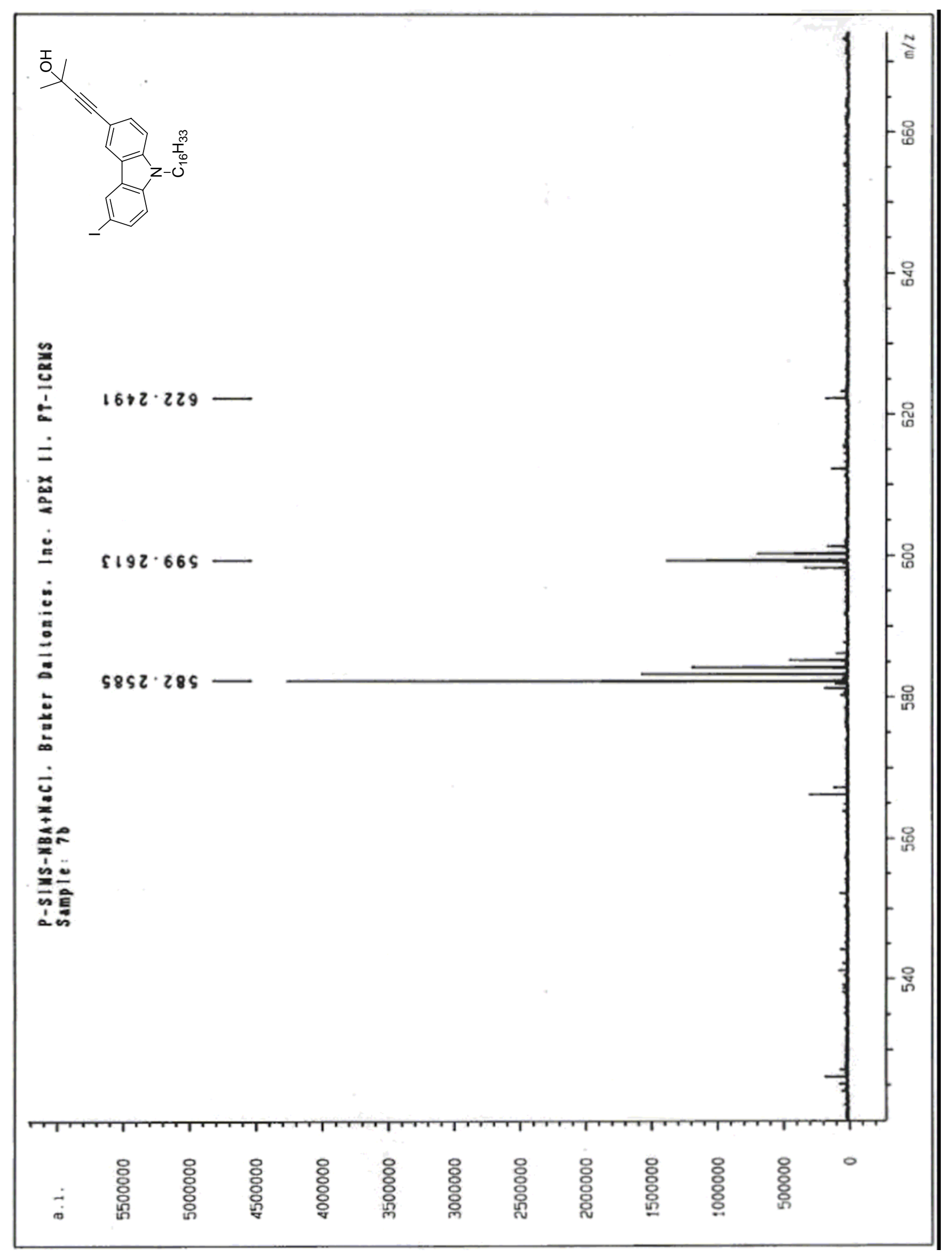

Figure S57: HRMS of 7b 


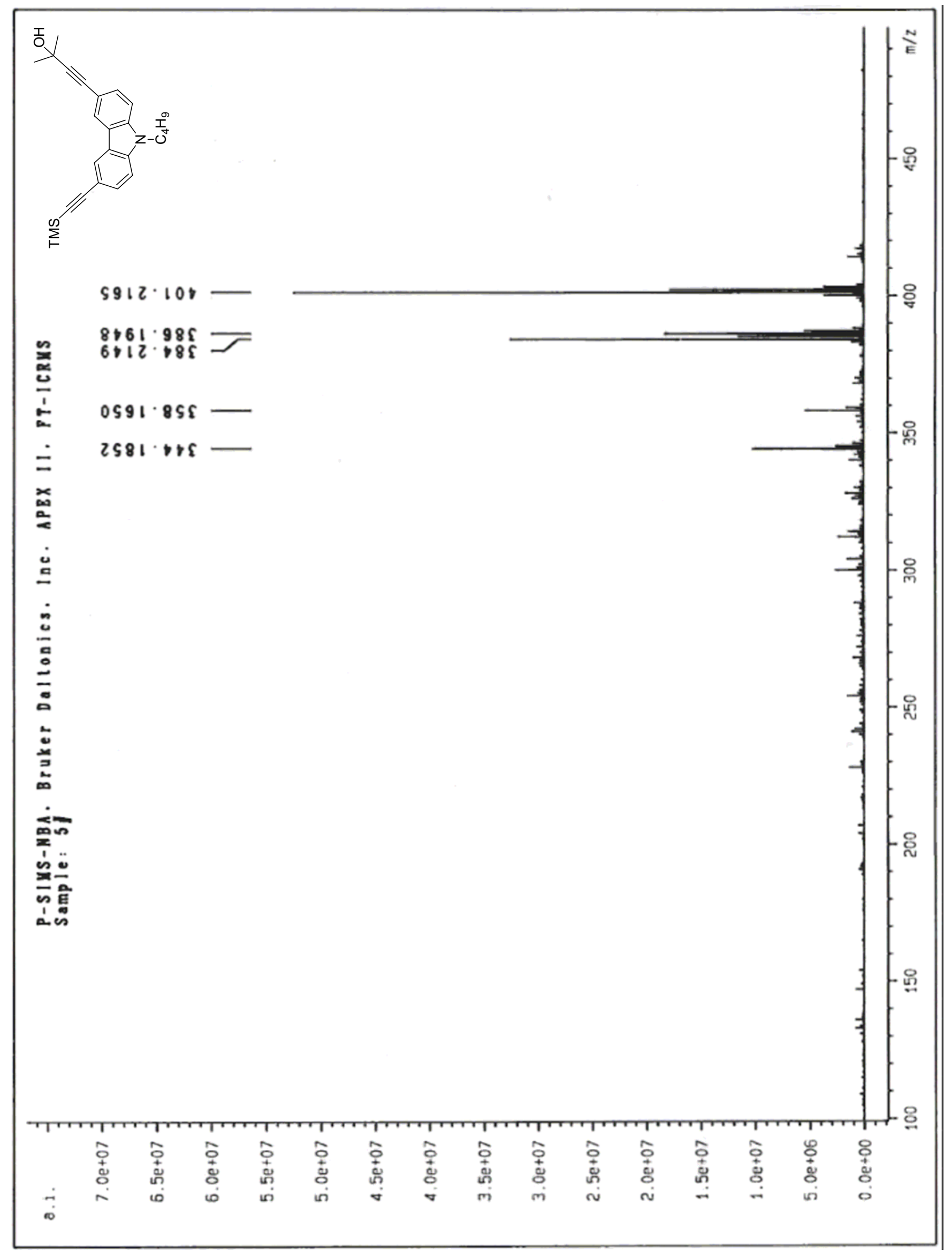

Figure S58: HRMS of 8a 


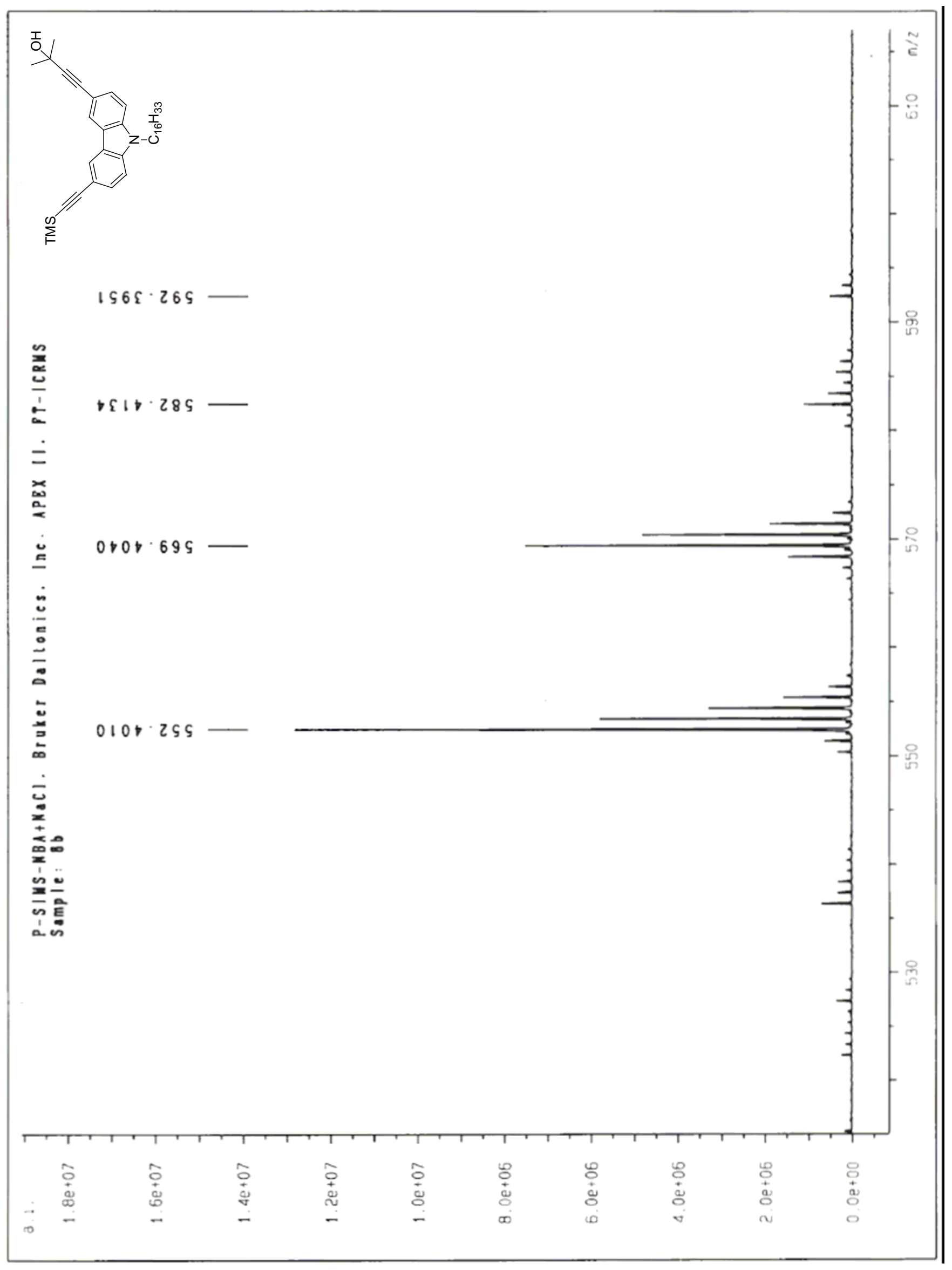

Figure S59: HRMS of $\mathbf{8 b}$ 


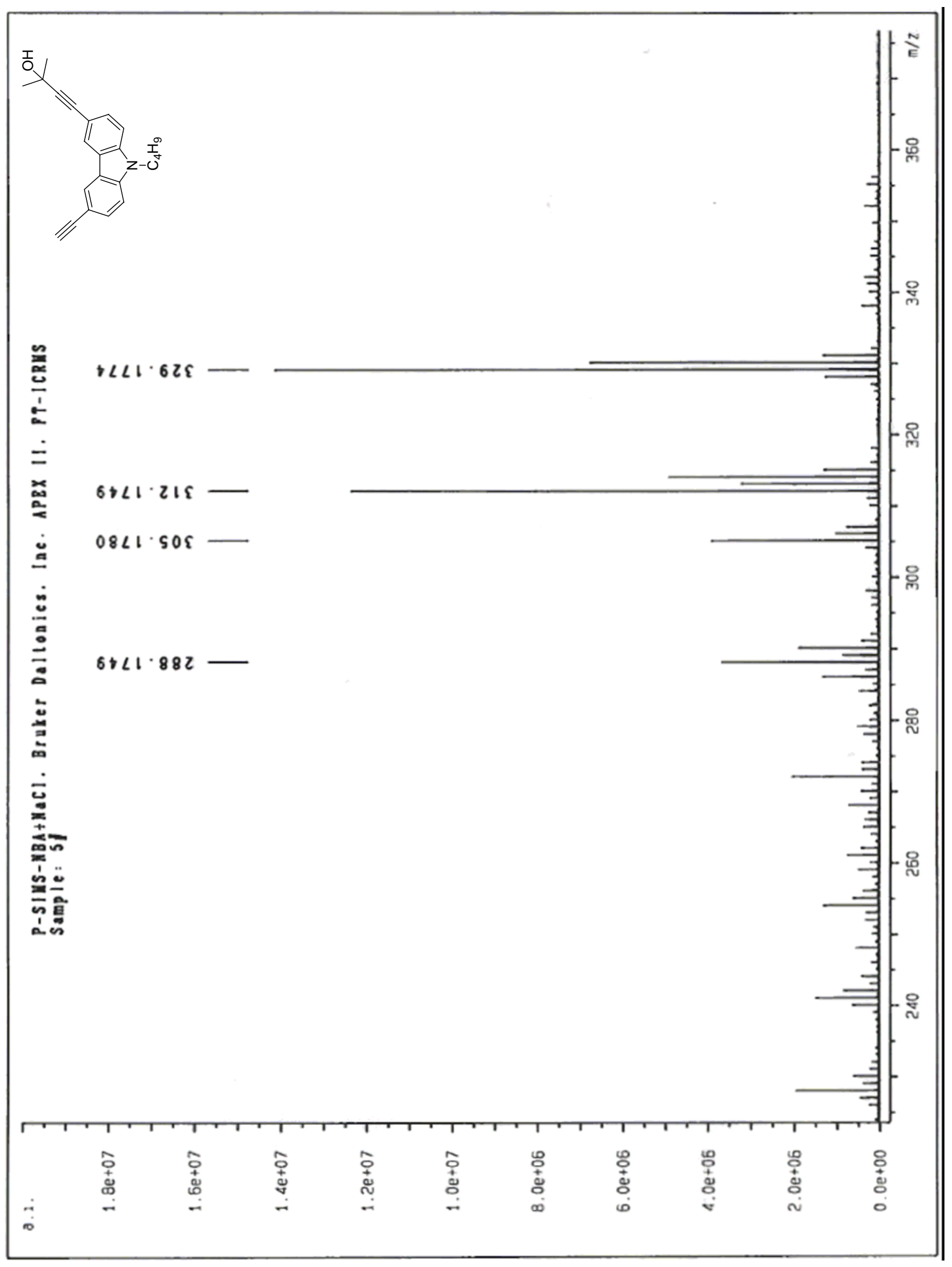

Figure S60: HRMS of 9a 


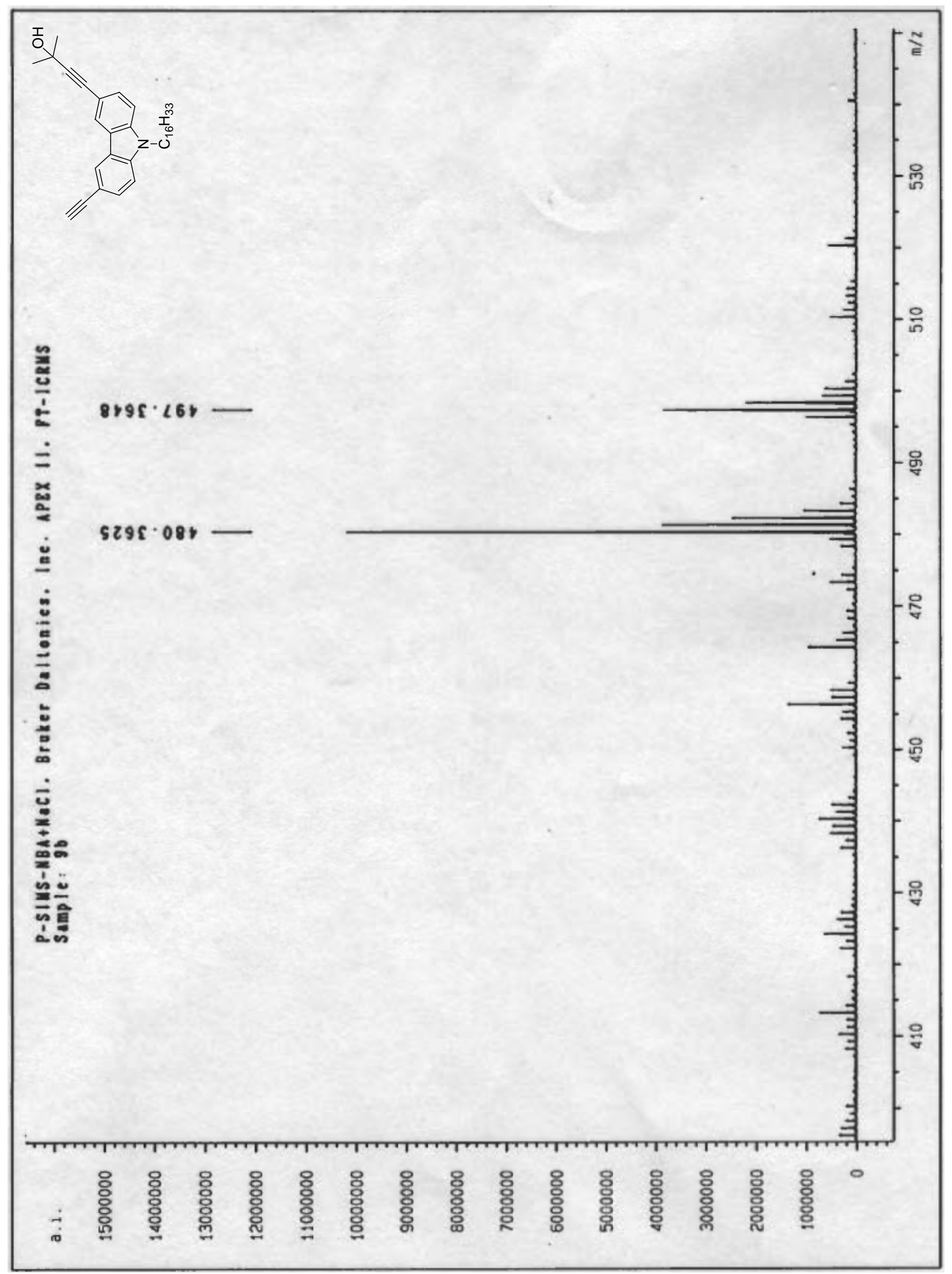

Figure S61: HRMS of 9b 


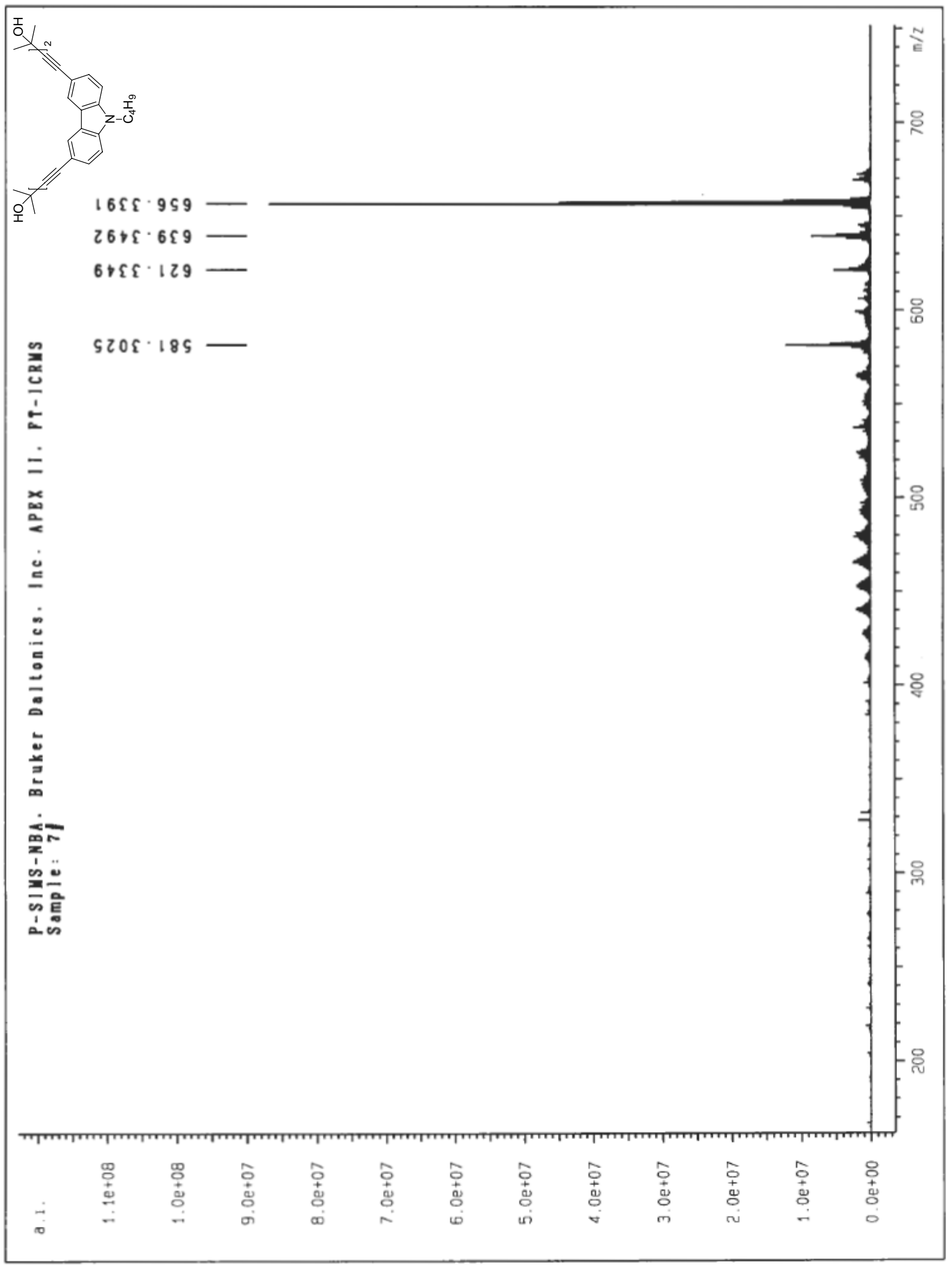

Figure S62: HRMS of 10a 


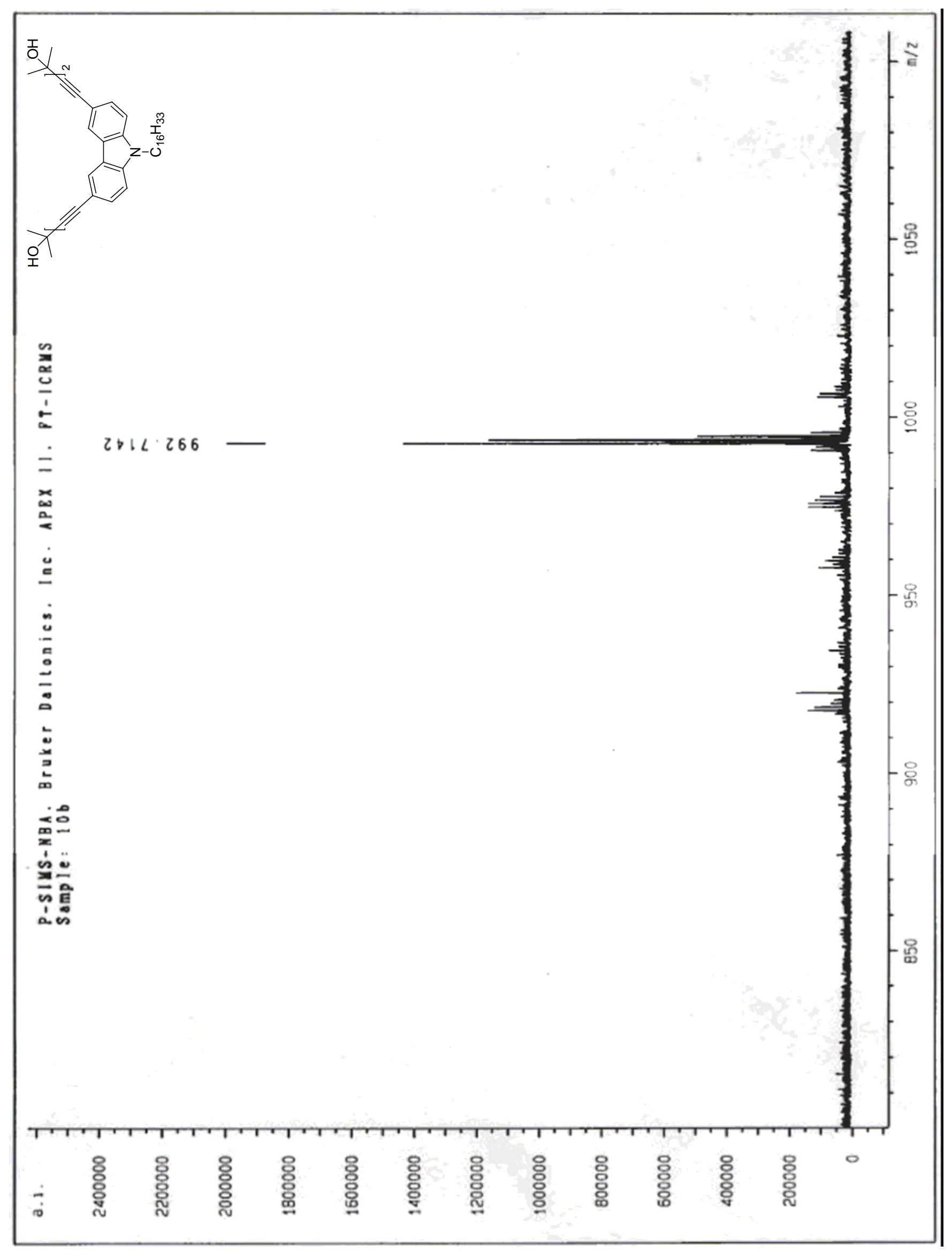

Figure S63: HRMS of 10b 


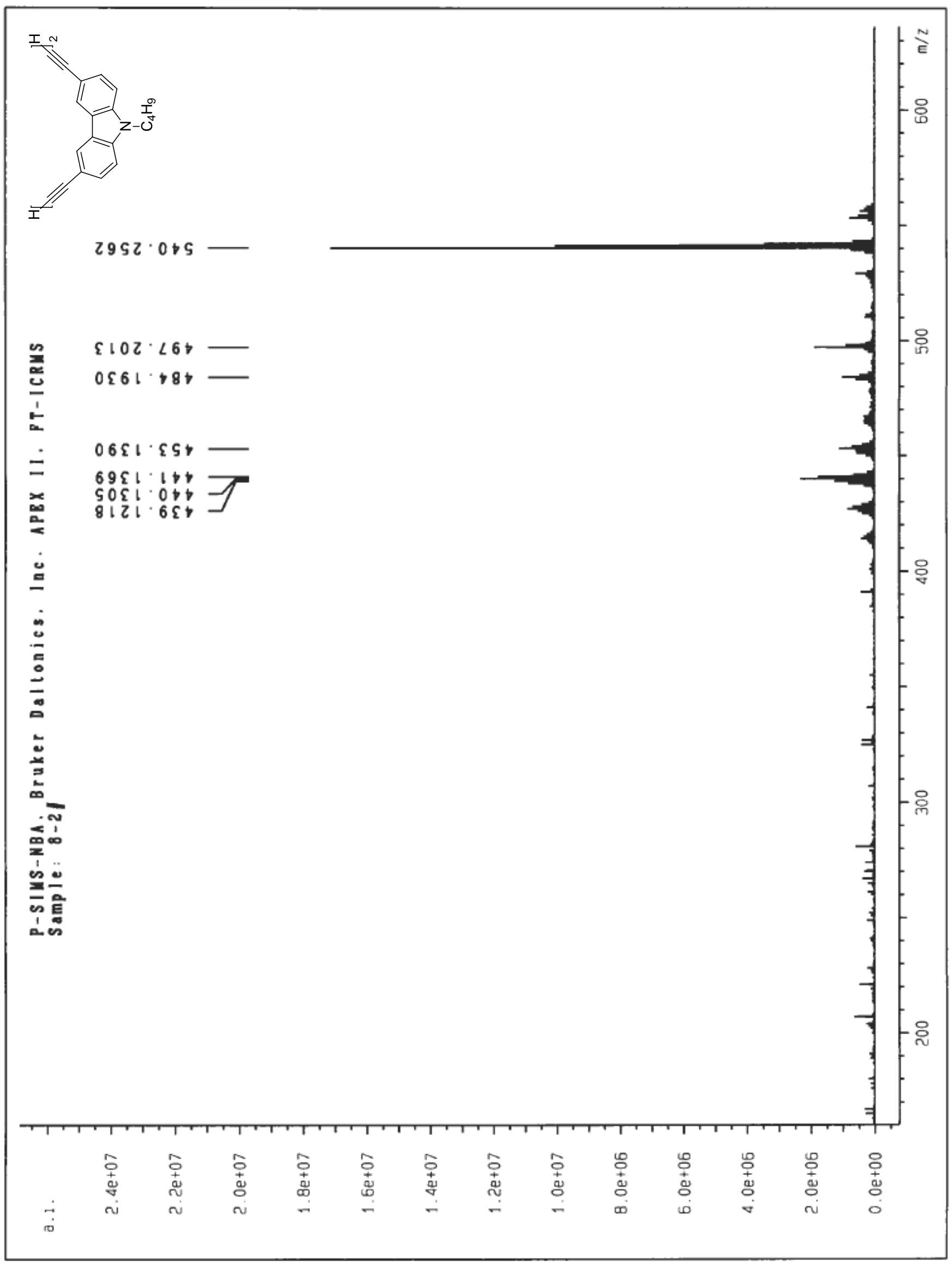

Figure S64: HRMS of 11a 


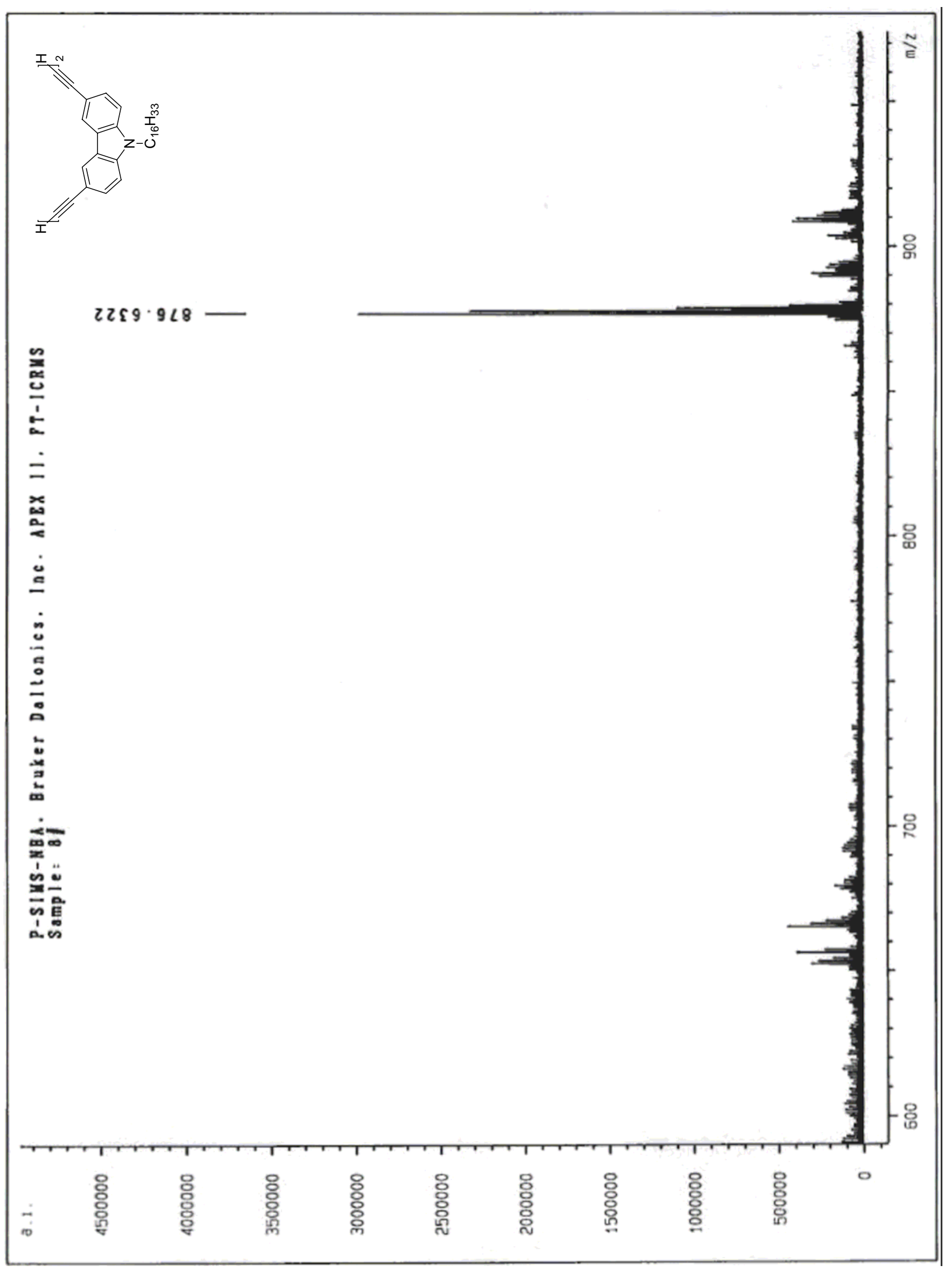

Figure S65: HRMS of 11b 


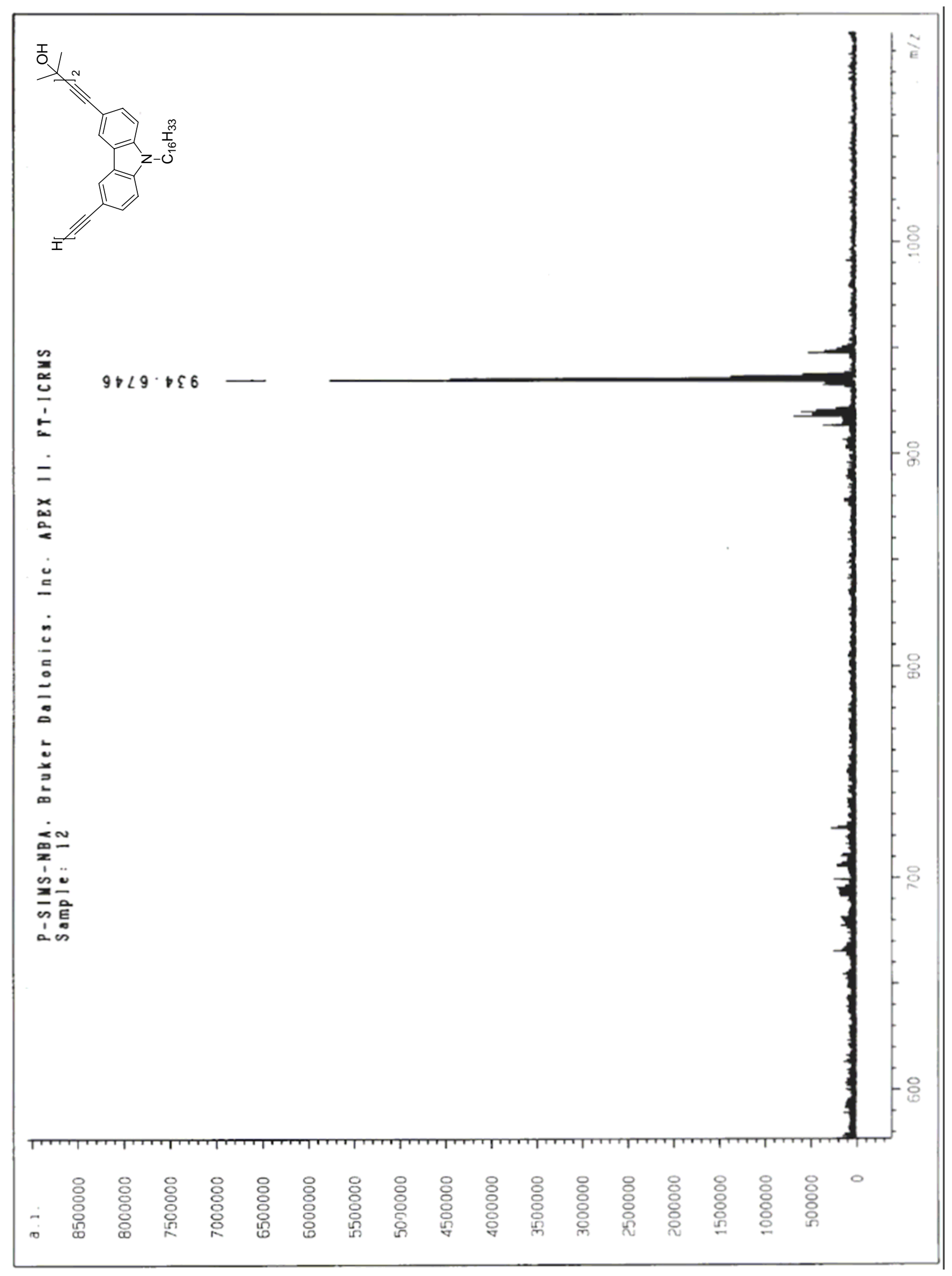

Figure S66: HRMS of 12 